\title{
Variational Quantum Approaches for Computing Vibrational Energies of Polyatomic Molecules
}

\author{
Joel M. Bowman * \\ Cherry L. Emerson Center for Scientific Computation and \\ Department of Chemistry, Emory University, \\ Atlanta, Georgia 30322, USA \\ Tucker Carrington Jr. ${ }^{\dagger}$ \\ Chemistry Department \\ Queen's University \\ Kingston, Ontario, K7L 3N6, Canada \\ Hans-Dieter Meyer $\ddagger$ \\ Theoretische Chemie, Physikalisch-Chemisches Institut, \\ Universität Heidelberg, Im Neuenheimer Feld 229, \\ 69120 Heidelberg, Germany
}

July 17,2008

${ }^{*}$ Corresponding author, Electronic mail: jmbowma@emory.edu, FAX:404-727-6628

${ }^{\dagger}$ Electronic mail: Tucker.Carrington@chem.queensu.ca

${ }^{\ddagger}$ Electronic mail: Hans-Dieter.Meyer@pci.uni-heidelberg.de 


\begin{abstract}
In this article we review state-of-the-art methods for computing vibrational energies of polyatomic molecules using quantum mechanical, variationally-based approaches. We illustrate the power of those methods by presenting applications to molecules with more than four atoms. This demonstrates the great progress that has been made in this field in the last decade in dealing with the exponential scaling with the number of vibrational degrees of freedom. In this review we present three methods that effectively obviate this bottleneck. The first important idea is the n-mode representation of the Hamiltonian and notably the potential. The potential (and other functions) are represented as a sum of terms that depend on a subset of the coordinates. This makes it possible to compute matrix elements, form a Hamiltonian matrix, and compute its eigenvalues and eigenfunctions. Another approach takes advantage of this multimode representation and represents the terms as sum of products. It then exploits the powerful multiconfiguration Hartree time dependent method to solve the time-dependent Schrödinger equation and extract the eigenvalue spectrum. The third approach we present uses contracted basis functions in conjunction with a Lanczos eigensolver. Matrix vector products are done without transforming to a direct-product grid. The usefulness of these methods is demonstrated for several example molecules, e.g., methane, methanol and the Zundel cation.
\end{abstract}




\section{Introduction}

The theory and computational modeling of molecular vibrations continues to be a central topic of interest in chemistry and in many related fields. In the broadest terms there are two aspects to this field. They are the molecular Hamiltonian, the sum of the kinetic energy operator (KEO), and the potential energy surface (PES), and the extraction of the vibrational information, i.e., eigenvalues, eigenfunctions, spectra, etc. from the Hamiltonian. This review will focus exclusively on so-called ab initio approaches to both aspects. That is, we will consider PESs that have been obtained from or heavily based on $a b$ initio electronic energies and we will only consider quantum mechanical approaches to the vibrational dynamics that are based on variational theory as opposed to perturbation theory. Examples of applications will be given to molecules with more than four atoms to indicate the current state-of-the-art.

In order to put this review in context it is useful to begin with a short and highly selective history of this quantum approaches to vibrational dynamics beginning in roughly the mid 1970s. It was at this time that variational approaches to obtain vibrational energies were undertaken using the rigorous Watson Hamiltonian in mass-scaled rectilinear normal modes [1]. Whitehead and Handy's [2] seminal work on triatomic molecules using this approach is certainly noteworthy. They used a direct-product representation of the wavefunction and employed a quartic force field for the potential. The approach requires matrix elements of the Hamiltonian in this basis and multidimensional quadratures over the potential. (Quartic force fields originated much earlier in the field in the context of standard Rayleigh-Schrödinger second order perturbation theory and were very much in focus during the 1970s and 80s. Their calculation from ab initio electronic energies was and continues to be an important aspect of the field $[3,4]$.)

Given any Hamiltonian describing the nuclear motion of $f$ modes the direct-product representation of the $f$-mode wavefunction is given by $\left\{\phi^{(1)}(1)\right\} \times$ $\left\{\phi^{(2)}(2)\right\} \cdots \times\left\{\phi^{(f)}(f)\right\}$. Each set of basis functions should be complete and orthonormal in the space of the mode. Typically they are eigenfunctions of a simple 1D Hamiltonian, e.g., a Harmonic or Morse oscillator Hamiltonian, free-rotor Hamiltonian, etc.

Without any further restrictions the size of the basis, and hence the order of the Hamiltonian matrix, is $O\left(\right.$ nbasis $\left.^{f}\right)$ where nbasis is the size of each single-mode basis. A typical value of nbasis is 10 and therefore, if direct linear algebra methods are used, the basis size, $O\left(10^{f}\right)$, limits this approach to $f=3$, i.e., triatomic molecules. Quadratures of the potential in this basis also show exponential scaling of $O\left(10^{f}\right)$. (Actually more quadrature points per degree of freedom are needed than the maximum quanta in the basis to obtain accurate quadratures.) This approach is now referred to as the Finite Basis Representation (FBR).

A direct-product, grid-based representation of the wavefunction, known as 
the Discrete Variable Representation (DVR), eliminates the need to perform multidimensional quadratures over the potential (since in this representation the potential matrix is diagonal). However, to achieve a comparable accuracy to an FBR more grid points than basis functions are generally required. (Note that FBR also has a different and very specific meaning in the context of the DVR, where the two representations are related to each other by a unitary transformation. Our usage of FBR above does not make reference to a DVR and we use FBR here with this more general meaning.)

Even with some method to contract the size of the direct-product basis/grids, e.g., using a maximum sum of quanta or eliminating grid points where the potential exceeds some maximum value, the scaling of this representation of the wavefunction remains essentially exponential in the number of degrees. For non-routine cases, e.g., isomerization, very high energy states may occur, requesting nbasis to be larger than 10, at least for some degrees of freedom, and restricting single-mode excitations may not be possible. In addition for these more challenging situations the choice of coordinates and thus the form of the Hamiltonian becomes a key consideration. These elements of the variational approach will recur throughout this review.

With respect to different choices of coordinates we note the pioneering variational calculations of Carter and Handy and co-workers using valence coordinates for triatomic molecules [5] and special classes of tetraatomic molecules $[6,7]$. A major aspect of this work was the derivation of the KEOs, which are quite complex. This effort resulted in two FBR-based codes RVIB3 (triatomics) and RVIB4 (tetraatomics) that are available through the CCP6 web site $[8]$.

There are several useful alternatives to direct-product representations of the wavefunction. One is based on a Hartree representation of the wavefunction, followed by CI methods analogous to those used in electronic structure theory. The development of Vibrational Self-Consistent field (VSCF) and Virtual space CI (VCI) approaches [9-12] (see also Sec. 7.4) using global or semi-global potential energy surfaces (not force fields) were reported, both in normal and curvilinear (Jacobi) coordinates for triatomic molecules. One of the earliest examples of this approach was to HCO where a global PES was used to obtain vibrational energies using the FBR initially in normal coordinates [13] and later in Jacobi coordinates [14], and where excellent agreement, as expected, from the two sets of calculations was seen. Also in the 1980s VSCF/VCI methods were used in a code, POLYMODE, [15], which was based on the Watson Hamitonian but which explicitly made use of quartic force fields in normal modes. This approach is quite attractive as matrix elements of the potential are analytical. This approach with many refinements has been further developed quite recently into a very useful computational tool [16].

Another very powerful alternative to the direct-product representation is a contraction method based on truncation-recoupling. In this approach eigenfunctions of subspace Hamiltonians are used to form multi-dimensional bases 
for final diagonalization. Carter and Handy were perhaps the first to develop these ideas for triatomic molecules in the FBR. Bowman and Gazdy [17] developed a more sophisticated version of this approach in the FBR (which is in the spirit of Light and co-workers version of truncation-recoupling in the DVR, described briefly below) where effective Hamiltonians for two degrees of freedom are obtained by averaging the full Hamiltonian over each basis function for the third degree of freedom.

For 3 degrees of freedom the simple version of this strategy is as follows. Let the full Hamiltonian be decomposed as

$$
\begin{aligned}
H & =H_{2 D}+H_{1 D}+V_{c}(1,2,3) \\
H_{2 D} & =T_{1}+T_{2}+V(1,2,0) \\
H_{1 D} & =T_{3}+V(0,0,3) \\
V_{c}(1,2,3) & =V(1,2,3)-V(1,2,0)-V(0,0,3)
\end{aligned}
$$

where $\mathrm{T}_{i}$ are the kinetic energy operators (assumed separable) and $\mathrm{V}$ is the full potential. Let the $2 \mathrm{D}$ eigenfunctions of $\mathrm{H}_{2 D}$, denoted $\psi_{m}^{2 D}(1,2)$, be obtained from a standard direct-product basis of say dimension $n_{1} \times n_{2}$ and let $\phi_{n}(3)$ be the eigenfunctions of $\mathrm{H}_{1 d}$. Then the truncated 3D basis is given by $\left\{\psi^{(2 D)}(1,2)\right\} \times\left\{\phi^{(3)}(3)\right\}$, where the number of $2 \mathrm{D}$ basis functions is much less than $n_{1} \times n_{2}$ and thus the dimension of this basis is much less than that of the underlying direct-product basis. A further advantage is that the $2 \mathrm{D}$ eigenfunctions contain two-mode correlation and are therefore a very good basis especially for cases where the third mode may be the least coupled of the three modes.

For a number of very challenging problems, perhaps best exemplified by the $\mathrm{HCN} / \mathrm{HNC}$ isomerization, neither normal coordinates nor valence coordinates are suitable to describe this large-amplitude motion. Even with better suited (atom-diatom Jacobi) coordinates the standard variational approaches in use the 1980s were not able to obtain well-converged energies for this system. Light and co-workers [18-20] introduced two seminal ideas that conquered this problem and greatly advanced the field. The first was so the so-called Discrete Variable Representation (DVR) of the Hamiltonian, based on a compact underlying quadrature scheme, and the second was the use of a sequential diagonalization in a subspace followed by a truncation-recoupling for the final diagonalization step. (In passing we note that an FBR using a "moveable basis" [21] was applied successfully to $\mathrm{HCN} / \mathrm{HNC}$ isomerization using an accurate PES [22].) A code based on the DVR approach for triatomic molecules, DVR3D, written by Tennyson and co-workers is available at the CCP6 web site $[8]$.

This general truncation-recoupling strategy has largely been responsible for the success in the 1990s and to the present in doing variational calculations of the vibrational energies of tetraatomic molecules. Perhaps one of the most notable successes of this strategy was to the challenging acetylene/vinylidene 
isomerization. In two approaches Jacobi coordinates in diatom-diatom $\mathrm{C}_{2}-\mathrm{H}_{2}$ coordinates were used in order to make maximum use of the permutationinversion symmetry $[23,24]$. Also the KEO is relatively simple in these coordiantes. In the work of Zou et al. [23] a 4D basis in the three angular variables plus $R$ (the distance between the $\mathrm{C}_{2}$ and $\mathrm{H}_{2}$ centers of mass) was combined with a $2 \mathrm{D}$ basis in the remaining diatom distances $\mathrm{r}_{C C}$ and $\mathrm{r}_{H H}$. This challenging system was also tackled with this and two other sets of curvilinear coordinates [25] in order to illustrate the differences in efficiency of the various coordinates for the different classes of eigenstates, i.e., acetylene-like, vinylidene-like and totally delocalized states. A code from this group [25] for tetraatomic molecules, WAVR4, is available at the CCP6 web site [8].

There are important differences between the contraction scheme of Carter and Handy and that of Light and co-workers (see section 5). In the scheme of Carter and Handy the contracted basis is obtained by computing eigensolutions of a small number of reduced dimensional Hamiltonians equal to the number of groups of coordinates. In the scheme of Light and co-workers the number of reduced Hamiltonians for which one must obtain eigensolutions is much larger and depends on the number of primitive basis functions. The scheme of Light et al. is not practical for moleules with more than four atoms. Computing and storing eigenvectors of all the reduced Hamiltonians becomes unpractical (see section 5).

Contracted basis functions are one way of dealing with the nbasis ${ }^{f}$ basis size problem emphasized above. Iterative eigensolvers are another. Although for a four-atom molecule it is impossible to use a nbasis ${ }^{f}$ direct product basis with a standard diagonalization method, it is possible to use it with an iterative eigensolver. See section 4 . Iterative eigensolvers enable one to compute a spectrum without storing or even computing Hamiltonian matrix elements. Iterative does not mean that energy levels are computed one by one, rather it means that they are computed from a series of matrix-vector products. Because matrix-vector products can be evaluated without first computing Hamiltonian matrix elements there is no need to do multi-dimensional integrals. Not surprisingly, it is advantgeous to use both contracted basis functions and iterative eigensolvers but to do so efficiently one must employ a good scheme for doing the matrix-vector products.

Finally, and although it is not the focus of this review, we note again that second order perturbation theory (PT), which has a longer history than variational theory, has been widely applied and developed with the use of quartic force fields. [26] Extensions of PT beyond second order have also been developed and have been widely applied, for example by Sibert and co-workers. [27, 28] Also, PT based on the VSCF Hamiltonian has been developed by Gerber and co-workers [29,30] and further developed and applied by Christiansen, [31-33] Benoit and co-workers [34-36] and Barone [37].

Our discussion so far has been focused on the time-independent picture. We now will briefly discuss the time-dependent approach as well. People 
became aware of the power of the time-dependent approach largely trough the seminal papers of Heller [38,39], (although the work of McCullough and Wyatt $[40,41]$ should also be mentioned). Heller's approach is very approximate but it made the time-dependent picture popular and shortly after the appearance of his papers new techniques, like integrators dedicated to the time-dependent Schrödinger equation [42], were developed. The excellent review of Kosloff [43] reflects the state of the art of accurate time-dependent methodologies twenty years ago.

The domain of time-dependent methods were mainly scattering or halfscattering (e. g. photo-dissociation) problems. Time-dependent methods also proved to be very useful for simulating low-resolution spectra, but the generation of high resolution spectra - the topic of the present review - required long propagation times making the time-dependent approach less efficient. The situation improved somewhat with the advent of filter diagonalization [44-49]. However, the calculation of eigenenergies seemingly remained the natural domain of methods based on the time-independent Schrödinger equation.

One of the advantages of the time-dependent approach becomes apparent when representing multi-dimensional wavefunctions. A time-dependent wavepacket, at each instant of time, is in general less structured and hence more easily approximated by parametrized functions or basis sets than a timeindependent eigenstate. For example, it is well known that time-dependent Hartree provides in general better approximate eigenenergies than time-independent Hartree (also known as VSCF). The multiconfiguration time-dependent Hartree (MCTDH) method, discussed in Sec. 7, is a powerful way to solve the time-dependent Schrödinger equation efficiently. For larger systems its high efficiency can compensate for the weakness of the time-dependent approach in computing vibrational energies. For the Zundel cation, $\mathrm{H}_{5} \mathrm{O}_{2}^{+}$, this is shown in Sec. 8.2.

A review of recent progress up to the present is the subject of the following sections. We begin with a review of representations of the potential and the kinetic energy operator in Sections 2 and 3, with a focus on molecules containing more than 4 atoms. We briefly describe eigensolvers in Sec. 4, followed by three sections describing approaches and codes based on variational solutions of the time-independent and time-dependent Schrödinger equation. Sect. 5 is focused on contracted iterative techniques for solving the the time-independent Schrödinger equation based on polyspherical coordinates. Section 6 . describes the approach based on the Watson Hamiltonian, the n-mode representation of the potential and the VSCF/VCI theory, already mentioned; however, it uses a new representation of the potential reviewed in the following section. The third approach, which is based on the time-dependent Schrödinger equation, is the Multi-Configuration-Time-Dependent Hartree method which is described in Sec. 7. Sec. 8 presents examples of applications of these methods to challenging molecules of more than four atoms. We conclude with a summary and short comments on possible future directions for the field. 


\section{Representations of the Potential}

If the number of vibrational coordinates is 6 (or less) it is practical to do full-dimensional quadratures of the potential, as is often done in the FBR, to compute the vibrational energies. It is also practical for this number of coordinates to store the potential on a $6 \mathrm{D}$ direct product grid and use a product (FBR or DVR) approach that avoids full-dimensional quadratures (see for example [50-56]). For larger molecules the dimensionality of the quadratures and the size of the product grid make these approaches currently impractical. Solutions to these two problems have been proposed. One, which addresses the quadrature problem, is to represent the potential in a fashion so that only quadratures of lower dimension than the full dimension are required. The other, which addresses the grid size problem, is to compute a reduced matrix, called $\mathbf{F}$, as explained in detail in Sec. 5 .

This section reviews two methods of representing the potential. The first (see Sec. 2.1) is the so-called n-mode representation. This general representation for use in vibrational calculations was proposed in 1997 [57] and in independent work in a more general context in 1998 [58]; the restricted 2-mode version appeared in 1996 [59] and was used in the context of second order perturbation theory. The second (see Secs. $2.2-2.6$ ) is a product representation of the potential. Such product forms, which may be generated by the potfit algorithm, are extensively used in MCTDH.

\section{$2.1 \quad$ n-mode representation}

In the n-mode representation (nMR) the potential $V(\mathbf{Q})$ is given by

$$
V(\mathbf{Q})=V^{(0)}+\sum_{i} V_{i}^{(1)}\left(Q_{i}\right)+\sum_{i j} V_{i j}^{(2)}\left(Q_{i}, Q_{j}\right)+\sum_{i j k} V_{i j k}^{(3)}\left(Q_{i}, Q_{j}, Q_{k}\right)+\cdots
$$

where the one-mode representation of the potential contains only $V_{i}^{(1)}\left(Q_{i}\right)$ terms, which are the cuts through the hyperspace of normal coordinates with just one coordinate varying at a time, the two-mode representation contains those terms plus the $V_{i j}^{(2)}\left(Q_{i}, Q_{j}\right)$ terms, etc. Each of the terms in each summation is an intrinsic p-mode potential, because they each vanish if one of the variables is zero. However, if full 2 or 3 -mode grids are used the above representation can still be used provided the overcounting of lower-dimensional grids embedded in higher dimensional ones is accounted for [57]. In order for this representation to be useful it must be truncated at a value of $n$ less than the total number of modes. In the original implementation of this representation in the code MULTIMODE [57], which uses VSCF/VCI methods to obtain eigenvalues and eigenfunctions of the exact Watson Hamiltonian, the maximum value of $\mathrm{n}$ was 4 ; the current maximum value in the code is 6 .

The n-mode representation has a number of useful features. First, matrix elements of the potential can be done relatively easily for up to a $6 \mathrm{MR}$ of the 
full potential. Second, each p-mode potential can be of arbitrary polynomial order thus eliminating the issues of negative divergences of say a quartic force field in the limit of large values of normal coordinates. Another feature of this representation of the potential is that convergence of vibrational energies obtained with increasing nMR can be systematically monitored. This important aspect of the nMR of the potential will be demonstrated in Sec. 8.1 for $\mathrm{CH}_{4}$.

Several approaches have been taken to obtain these p-mode potentials. If one has a global or semi-global PES they can be obtained directly from it. However, they can also be obtained directly from $a b$ initio calculations. For 2mode potentials this is feasible, but for larger p-mode potentials this becomes problematic as the number of grid point grows rapidly. For example even for tetraatomic molecules there are 203 -mode grids and if one needed say 8 electronic energies per mode this would amount to 10, 000 energies. For the 15 4-mode grids 61, 000 electronic energies would be needed which is bordering on impractical. There are ways to mitigate this bottleneck. One is to use sparse data on the various grids and then to interpolate the energy on each p-mode grid. For example if one uses 5 energies per mode the above numbers become 2500 and 9375 for 3 and 4 mode grids. This strategy was proposed by Carter et al. [60] who suggested least-squares polynomial fitting or multidimensional spline intepolation as well as a less well-known approach which makes explicit use of gradient data, namely reduced Hermite interpolation. Even using interpolation the number of electronic energies needed for 3-mode grids can be quite large for molecules larger than tetraatomics. One effective so-called "multi-resolution" approach to deal with this has been proposed. In this approach lower levels of $a b$ initio theory are used for say the 3-mode grids compared to the 2-mode ones [61,62]. Another strategy is to consider only a subset of p-mode potentials, as was done in recent applications to the 12-atom molecule n-methyl acetamide [63,64].

Defining the mode terms to minimize the error in all of configuration space is another technique to obtain the p-mode potentials. Using the mode terms defined after Eq. (2.1) is equivalent to minimizing the error along 1D slices, 2D surfaces etc. Instead, it is possible to use a random sampling high dimensional model representation [65]. In this case the different terms are not associated with different grids but the error is minimized for a set of points drawn from some suitable probability distribution and all mode terms are fit from a single set of ab initio points. For example, one can choose the probability distribution to favour low-lying regions of the potential and use neural networks (NNs) to fit the mode terms. Details are given in Ref. [66]. Beginning with the one-mode term, the mode terms are determined sequentially. A fit for the s-mode term is built by fitting a NN to the difference of the potential and the sum of the previously determined mode terms. Note that unlike the original approach of Ref. [67] the mode terms can be determined without doing multi dimensional integrals by Monte Carlo methods. For $\mathrm{HOOH}$, using this approach we are able to determine all of the four-mode terms from about 10000 points. Levels 
computed on the surface are good to about $1 \mathrm{~cm}^{-1}$.

We conclude this subsection by noting that the n-mode representation can also be applied to any multidimensional function. In particular it has been applied to the components of the dipole moment, which is needed in the calculation of the IR spectrum. This has been implemented in MULTIMODE, where matrix elements of a property can be evaluated, using an n-mode representation of that property [68] .

\subsection{Separable representation of potential: The product form}

As noted already, the set-up of the Hamiltonian matrix can become a major part of the work to be performed when investigating molecular vibrations. Multi-dimensional integrals have to be done and when there are $f=6$ or more degrees of freedom (DOF) then these integrals are difficult to perform. The computation of the matrix elements of $\hat{H}$ is considerably simplified if the Hamiltonian is of product form, i. e. if it can be written as

$$
\hat{H}=\sum_{r=1}^{s} c_{r} \prod_{\kappa=1}^{f} \hat{h}_{r}^{(\kappa)}
$$

where $\hat{h}_{r}^{(\kappa)}$ operates on the $\kappa$-th DOF only and where $c_{r}$ is a number. Then multi-dimensional integrals can be written as a sum of products of one-dimensional integrals,

$$
\left\langle\Phi_{J}|\hat{H}| \Phi_{L}\right\rangle=\sum_{r=1}^{s} c_{r}\left\langle\chi_{j_{1}}^{(1)}\left|\hat{h}_{r}^{(1)}\right| \chi_{l_{1}}^{(1)}\right\rangle \ldots\left\langle\chi_{j_{f}}^{(f)}\left|\hat{h}_{r}^{(f)}\right| \chi_{l_{f}}^{(f)}\right\rangle .
$$

Here $\chi_{j}^{(\kappa)}$ denotes the $j$-th basis function of the basis for the $\kappa$-th DOF. Often these basis functions are chosen as DVR functions, which simplifies the evaluation of matrix elements of local operators. The multi-dimensional basis functions (configurations) $\Phi_{J}$ are products of the one-dimensional ones, i.e. we assume a direct product basis. The composite index $J=\left(j_{1}, \cdots, j_{f}\right)$ collects all indices of one configuration.

As one-dimensional integrals are done very fast, the computation of the Hamilton matrix elements according to Eq. (4) is much faster than doing multi-dimensional integrals. The storage requirements are also much smaller. However, one may ask how realistic it is to assume a Hamiltonian of product form. Well, kinetic energy operators (KEO) are mostly of product form. In particular when using polyspherical coordinates, the KEO is of product form. The potential energy surface (PES), however, is often not of product form, although a Taylor expansion of the potential as well as many model potentials are of product form. Hence a polynomial fit of the potential - when done in the same coordinates as the dynamical calculation - is of desired product 
form. In the following we will discuss a method, called potfit [69-71], which allows to transform a general PES to product form, while controlling the error which is introduced by this re-fitting procedure.

Before we discuss potfit we remark that several methods for calculating vibrational energies, e. g., RVIB4 [7,8], rely on product form. For MCTDH, where the integrals have to be performed at every time-step, the product form is vital (except when using the CDVR method of U. Manthe [72]). Hence potfit was developed for MCTDH, but it can certainly be useful for other quantum dynamical methods as well.

\subsection{The POTFIT algorithm}

A direct way to the product form is an expansion in a product basis. Hence we approximate some given potential $V$ by

$$
V^{\mathrm{app}}\left(q^{(1)}, \ldots, q^{(f)}\right)=\sum_{j_{1}=1}^{m_{1}} \ldots \sum_{j_{f}=1}^{m_{p}} C_{j_{1} \ldots j_{f}} v_{j_{1}}^{(1)}\left(q^{(1)}\right) \ldots v_{j_{f}}^{(f)}\left(q^{(f)}\right),
$$

where $q^{(\kappa)}$ is the coordinate of the $\kappa$-th DOF. The basis functions $v_{j_{\kappa}}^{(\kappa)}\left(q^{(\kappa)}\right)$ are called single-particle potentials (SPP). The expansion orders, $m_{\kappa}$, must be chosen large enough to achieve an accurate expansion. On the other hand they should be as small as possible, because the numerical effort of the integral calculation scales linearly with the number of potential terms, i. e. with the product of the expansion orders. Hence both the expansion coefficients and the SPPs should be optimized to provide the best approximate potential for a given set of expansion orders.

Before we turn to analyze this optimization problem, we simplify the problem somewhat. When DVRs are used to represent the wavefunctions, one needs to know the potential only at grid points. This allows us to work in finite dimensional discrete vector spaces. The full potential is now represented by a tensor

$$
V_{i_{1} \ldots i_{f}}=V\left(q_{i_{1}}^{(1)}, \ldots, q_{i_{f}}^{(f)}\right)
$$

where $q_{i}^{(\kappa)}$ denotes the coordinate of the $i$-th grid point of the $\kappa$-th grid. The approximate tensor is written as

$$
V_{i_{1} \ldots i_{f}}^{\mathrm{app}}=\sum_{j_{1}=1}^{m_{1}} \ldots \sum_{j_{f}=1}^{m_{f}} C_{j_{1} \ldots j_{f}} v_{i_{1} j_{1}}^{(1)} \ldots v_{i_{f} j_{f}}^{(f)},
$$

with $v_{i j}^{(\kappa)}=v_{j}^{(\kappa)}\left(q_{i}^{(\kappa)}\right)$. The SPPs are assumed to be orthonormal on the grid, $\sum_{i} v_{i j}^{(\kappa)} v_{i \ell}^{(\kappa)}=\delta_{j \ell}$. Throughout this section we will use the letters $i$ and $k$ to label grid points and $j$ and $\ell$ to label SPPs. 
The task is now to determine optimal coefficients and SPPs. To this end we minimize

$$
\Delta^{2}=\sum_{i_{1}=1}^{N_{1}} \ldots \sum_{i_{f}=1}^{N_{f}}\left(V_{i_{1} \ldots i_{f}}-V_{i_{1} \ldots i_{f}}^{\mathrm{app}}\right)^{2}=\sum_{I}\left(V_{I}-V_{I}^{\mathrm{app}}\right)^{2}
$$

where $I$ denotes a composite index which runs over all grid points. Minimizing $\Delta^{2}$ by varying only the coefficients yields

$$
C_{j_{1} \ldots j_{f}}=\sum_{i_{1}=1}^{N_{1}} \ldots \sum_{i_{f}=1}^{N_{f}} V_{i_{1} \ldots i_{f}} v_{i_{1} j_{1}}^{(1)} \cdots v_{i_{f} j_{f}}^{(f)}
$$

i. e. the coefficients are given by overlap.

More difficult is to find optimal SPPs. Within potfit one defines the SPPs as eigenvectors of the potential density matrices

$$
\varrho_{k k^{\prime}}^{(\kappa)}=\sum_{I}^{\kappa} V_{i_{1} \ldots i_{\kappa-1} k i_{\kappa+1} \ldots i_{f}} V_{i_{1} \ldots i_{\kappa-1} k^{\prime} i_{\kappa+1} \ldots i_{f}},
$$

where the upper index $\kappa$ at the summation symbol indicates that the summation is not over the $\kappa$-th DOF. The eigenvectors are ordered according to their eigenvalues, i. e. one neglects SPPs with small eigenvalue and hence small contribution to the potential. This approach is known to be optimal for the two-dimensional case [73]. For the general $f$-dimensional case $(f>2)$, potfit is not optimal but provides SPPs which are close enough to the optimal ones to be useful. Fully optimal SPPs can be found - in principle - by minimizing $\Delta^{2}$, Eq. (8), with the aid of a general minimization procedure, e. g. simulated annealing. But such a procedure is much too costly.

The error of the approximate potential is checked by comparing it with the exact potential on the full product grid. But a simple estimate of the error based on the eigenvalues, $\lambda_{j}$, of the potential density matrices can also be given.

$$
\Delta^{2} \leq \sum_{\kappa=1}^{f} \sum_{j=m_{\kappa}+1}^{N_{\kappa}} \lambda_{j}^{(\kappa)}
$$

This estimate is very useful for determining appropriate expansion orders. It also shows that the $\mathrm{L}^{2}$ error vanishes uniformly with increasing expansion orders. If all $m_{\kappa}=N_{\kappa}$ one recovers the exact potential on the grid points.

\subsection{Contraction over one degree of freedom}

The number of expansion terms, $s=\prod_{\kappa=1}^{f} m_{\kappa}$ appearing in Eq. (7), should be as small as possible, because this number determines the effort of the integral calculation using this product-form potential. At virtually no cost one can 
reduce the number of expansion terms by one expansion order $m_{\kappa}$. To this end we define contracted expansion coefficients

$$
D_{j_{1} \ldots j_{\kappa-1} i_{\kappa} j_{\kappa+1} \ldots j_{f}}^{(\kappa)}=\sum_{j_{\kappa}=1}^{m_{\kappa}} C_{j_{1} \ldots j_{f}} v_{i_{\kappa} j_{\kappa}}^{(\kappa)} .
$$

As the expansion order $m_{\kappa}$ will no longer appear in the working equation for $V^{\text {app }}$, one may set $m_{\kappa}=N_{\kappa}$ and use the full set of SPPs for this particular DOF. In this case one performs a unitary transformation on the $\kappa$-th index of the potential to obtain the coefficient $C$, and then performs the inverse unitary transformation on the $\kappa$-th index of the coefficient. Hence effectively there is no transformation on the $\kappa$-th index and $D^{(\kappa)}$ is conveniently computed as

$$
D_{j_{1} \ldots j_{\kappa-1} i_{\kappa} j_{\kappa+1} \ldots j_{f}}^{(\kappa)}=\sum_{I}^{\kappa} V_{i_{1} \ldots i_{f}} v_{i_{1} j_{1}}^{(1)} \cdots v_{i_{\kappa-1} j_{\kappa-1}}^{(\kappa-1)} v_{i_{\kappa+1} j_{\kappa+1}}^{(\kappa+1)} \cdots v_{i_{f} j_{f}}^{(f)} .
$$

Note that the $\kappa$-th potential density matrix and the $C$-tensor are no longer needed. Using contraction, the approximate potential is written as

$$
\begin{aligned}
V_{i_{1} \ldots i_{f}}^{\mathrm{app}}= & \sum_{j_{1}=1}^{m_{1}} \ldots \sum_{j_{\kappa-1=1}}^{m_{\kappa-1}} \sum_{j_{\kappa+1=1}}^{m_{\kappa+1}} \ldots \sum_{j_{f}=1}^{m_{f}} D_{j_{1} \ldots j_{\kappa-1} i_{\kappa} j_{\kappa+1} \ldots j_{f}}^{(\kappa)} \\
& \times v_{i_{1} j_{1}}^{(1)} \cdots v_{i_{\kappa-1} j_{\kappa-1}}^{(\kappa-1)} v_{i_{\kappa+1} j_{\kappa+1}}^{(\kappa+1)} \cdots v_{i_{f} j_{f}}^{(f)} .
\end{aligned}
$$

This contraction over the $\kappa$-th DOF is a very helpful trick as it substantially reduces the numerical effort of the following integral calculation without affecting the accuracy of the product expansion. One should contract over that DOF which otherwise would require the largest expansion order.

\subsection{Including weights}

The inclusion of weights, i. e. minimizing $\Delta^{2}=\sum_{I}\left(V_{I}-V_{I}^{\text {app }}\right)^{2} w_{I}^{2}$, is often inevitable for obtaining an accurate product representation of the physically relevant part of the potential without going to high expansion orders. The inclusion of separable weights,Se $w_{I}=w_{i_{1}}^{(1)} \cdots w_{i_{f}}^{(f)}$, is very simple [69], but separable weights are not so helpful and hence not discussed here. The inclusion of non-separable weights unfortunately leads to complicated equations of little use. There is, however, a nice trick $[70,71]$, which allows one to emulate non-separable weights by an iterative procedure. To this end we introduce a reference potential $V^{\text {ref }}$ such that the weighted difference between the potential and its product representation is identical to the difference between the reference potential and the product representation

$$
\left(V_{I}-V_{I}^{\mathrm{app}}\right) w_{I}=V_{I}^{\mathrm{ref}}-V_{I}^{\mathrm{app}} .
$$


Then one simply can "potfit" the reference potential to obtain a product representation, $V^{\text {app }}$, which is (almost) optimal with respect to the weighted sum of squared differences. Obviously, $V^{\text {ref }}$ is given by

$$
V_{I}^{\mathrm{ref}}=w_{I} V_{I}+\left(1-w_{I}\right) V_{I}^{\mathrm{app}} .
$$

The definition of the reference potential depends on $V^{\text {app }}$ which in turn depends on the reference potential. Hence, the equations must be solved iteratively. One first potfits $V$ and evaluates the reference potential. Then the reference potential is potfitted and with the new $V^{\text {app }}$ a new reference potential is built. The process is iterated until some break-off criterion is satisfied.

When emulating non-separable weights we always have used a special form of the weights. The weights are set to one within the so called relevant region and are zero otherwise. The relevant region is usually defined by an energy criterion, i. e. it is the region where the potential is lower than some suitably chosen energy threshold. Restrictions on coordinates can be set as well when defining the relevant region. With such a definition of the weights, i. e. zero or one, Eq. (16) has a vivid interpretation. The reference potential is the original potential within the relevant region and the fitted potential otherwise. Moreover, with this choice of the weights we always observed a lowering of the weighted $\mathrm{L}^{2}$ error with each iteration.

\subsection{Computational effort and memory requirements}

The potfit expansion was introduced to reduce the numerical labor when evaluating the integrals. Consider the computation of the matrix element $<\Phi_{J}|V| \Phi_{L}>$. Doing this integral on the primitive grid requires $N^{f}$ multiplications. (Here we assume, for sake of simplicity, that all DOFs have the same number of grid points, $N_{\kappa}=N$.) Doing the integral with a potfit expansion requires $s f N$ multiplications. The number of potential terms is, due to contraction, $s=m^{f-1}$, where, similar to above, $m_{\kappa}=m$ is assumed. The gain is hence

$$
\operatorname{gain}_{\mathrm{CPU}}=\frac{1}{f}\left(\frac{N}{m}\right)^{f-1} .
$$

A potfit expansion does not only speed-up the calculation, it also compacts the representation of the potential leading to a much lower memory demand. The full potential consists of $N^{f}$ data points, whereas a potfit expansion, Eq. (14), takes $N m^{f-1}+N m(f-1)$ data points. For $f>2$ the second term is negligible in comparison with the first one, and one arrives at a memory gain

$$
\text { gain }_{\text {mem }}=\left(\frac{N}{m}\right)^{f-1} .
$$

As an example let us consider a 6D problem where each DOF is represented by 25 grid points. Assuming $m=6(m=5)$, i. e. 7776 (3125) potfit terms after 
contraction, one has a CPU-gain of 209 (521), which is a quite remarkable speed-up. The potential consists of $N^{f}=2.4 \times 10^{8}$ points and requires $1.8 \mathrm{~GB}$ of storage. The potfit consumes only $1.5 \mathrm{MB}(615 \mathrm{~KB})$. A potfit representation is hence very compact. This is an important feature when turning to larger systems where the potential evaluated at the grid points ceases to fit into memory. Unfortunately potfit cannot solve this problem. Although the potfit representation is very compact, to arrive at this representation one has to perform sums over all grid points, see Eqs. $(10,13)$. Hence, using today's workstations, potfit is limited to problems with less than $10^{9}$ grid points, i. e. in general to systems with at most six or seven degrees of freedom. One way out of this dilemma is to switch to a (in general more approximate) n-mode representation (see Sec. 2.1). One may then potfit the n-mode terms.

When the PES is build from scratch one may fit the ab-initio points by a model function which is of product form. As already noted, polynomial fits are of product form. New ideas on high dimensional potential fitting in product form using neural network techniques are recently discussed by Manzhos and Carrington [74].

Finally we note that in MCTDH potfit is used with a slight modification. MCTDH (see Sec. 7.2) makes use of mode combination and it is thus wellfounded to expand the potential in the same combined modes. The changes to the above equations are trivial. One merely has to replace the number of degrees of freedom, $f$, by the number of particles, $p$, and to interpret $N_{\kappa}$ as the number of points of the combined grid of the $\kappa$-th particle.

\section{Representations of the kinetic energy operator}

One must choose coordinates to use for the kinetic energy operator (KEO). Basis functions are almost always functions of the coordinates in terms of which the KEO is represented. As long as the transformation between the coordinates used for the KEO and the coordinates used for the potential is known, the potential can be a function of any (even redundant) coordinates.

In this section we focus on two quite different choices for coordinates, one is rectilinear normal coordinates and the other is curvilinear polyspherical coordinates. We point out the advantages and disadvantages of each and explain why each set is very much in use today. We consider normal coordinates first.

As noted already, early variational calculations of vibrational levels of triatomic molecules were done in rectilinear normal coordinates [2] at least in part because the exact (Watson) Hamiltonian in these coordinates was wellknown [1]. The Watson KEO (atomic units are used throughout this article) is

$$
\hat{K}_{W}=\frac{1}{2} \sum_{\alpha, \beta}\left(\hat{J}_{\alpha}-\hat{\pi}_{\alpha}\right) \mu_{\alpha \beta}\left(\hat{J}_{\beta}-\hat{\pi}_{\beta}\right)-\frac{1}{2} \sum_{k} \frac{\partial^{2}}{\partial Q_{k}^{2}}-\frac{1}{8} \sum_{\alpha} \mu_{\alpha \alpha},
$$


where

$$
\mu_{\alpha \beta}=\left(\mathbf{I}^{\prime-1}\right)_{\alpha \beta} ; \quad \mathbf{I}_{\alpha \beta}^{\prime}=\mathbf{I}_{\alpha \beta}+\sum_{k, l, m} \zeta_{k m}^{\alpha} \zeta_{l m}^{\beta} Q_{k} Q_{l}
$$

and where $I_{\alpha \beta}$ is the inertia tensor and $\zeta_{k m}^{\alpha}$ are Coriolis parameters defined for example in Ref. [1]. The vibrational angular momentum terms, $\pi_{\alpha}$, are

$$
\pi_{\alpha}=-i \sum_{k, l} \zeta_{k l}^{\alpha} Q_{k} \frac{\partial}{\partial Q_{l}}
$$

Note that we include the last "Watson" term in the expression for the KEO even though it does not contain differential operators.

The generality of the Watson KEO and thus the full Hamiltonian, which is just the sum of the KEO plus the potential $V$, was and is a major advantage and was a motivating factor for the development of variational codes based on it. An early example of such a code is POLYMODE [15] which was written in the mid 1980s and which uses quartic force fields. A number of other codes now exist based on the exact Watson Hamiltonian and also making use of the n-mode representation of the potential described in the previous section $[57,61,62,75-81]$.

The $\boldsymbol{\pi}^{\boldsymbol{t}} \boldsymbol{\mu} \boldsymbol{\pi}$ term causes non-separablity of the exact Watson KEO, even for $J=0$. In MULTIMODE [57,75-77] marix elements of this term are computed by representing the $\boldsymbol{\mu}$ matrix elements with an n-mode expansion analogous to the one used for the potential, but with an order smaller than that used for $V$. The $\boldsymbol{\pi}^{t} \boldsymbol{\mu} \boldsymbol{\pi}$ terms are of the order of the rotation constants, and thus are generally small compared to other terms in the KEO and in $V$. Thus, in numerous applications in the literature these terms are neglected. In practice one can accurately treat the $\boldsymbol{\pi}^{t} \boldsymbol{\mu} \boldsymbol{\pi}$ term, as done in MULTIMODE. If the basis is so large that the matrix representing $\boldsymbol{\pi}^{\boldsymbol{t}} \boldsymbol{\mu} \boldsymbol{\pi}$ cannot be stored in memory then matrix-vector products must be computed term by term and the fact that there are many $\boldsymbol{\pi}^{t} \boldsymbol{\mu} \boldsymbol{\pi}$ terms slows the calculation.

There are well-known limitations of normal coordinates and thus to the Watson Hamiltonian. First, the above KEO for non-linear molecules is singular at linear shapes where $\boldsymbol{\pi}^{\boldsymbol{t}} \boldsymbol{\mu} \boldsymbol{\pi}$ is singular. If a molecule approaches linear geometries this can significantly affect results of variational calculations. Carter and Bowman investigated this in detail for highly excited states of $\mathrm{H}_{2} \mathrm{O}$, which has an energy at the linear geometry of roughly $11100 \mathrm{~cm}^{-1}$ above the minimum. They examined the deviation of the energies of highly excited pure bend overtone states from exact results and found deviations ranging from 0.3 to $200 \mathrm{~cm}^{-1}$ for the third overtone to the 6th overtone [82]. For larger non-linear molecules this is of less concern, of course. Second,there is not a one to one mapping between points in normal coordinate space and molecular shapes. A simple example: points in normal coordinate space represent molecules with negative bond lengths. Third, normal coordinates describe large amplitude motions poorly and therefore basis functions that are 
functions of normal coordinates do not compactly represent wavefunctions of molecules undergoing large amplitude motion. This is in part due to the fact that normal coordinates are defined with respect to a single reference configuration. The fact that normal coordinates are rectilinear (and not curvilinear) also plays a role. There are, however, cases for which one can compute, using normal coordiantes, accurate tunneling splittings in multidimensions, e.g., for $\mathrm{H}_{3} \mathrm{O}^{+}$[83] and $\mathrm{NH}_{3}$ [84]. In these cases saddle point normal modes were used since they span the two symmetric minima and the saddle point separating them.

It should be pointed out that reaction path [85] (and reaction surface [86]) Hamiltonians enable one to overcome these disadvantages by using one or more curvilinear coordinates and normal coordinates defined with respect to displacements from a flexible reference. The associated KEOs are complex but there is a version of MULTIMODE based on this Hamiltonian and the n-mode representation of the potential and it has been used successfully in numerous applications [87-91].

For ro-vibrational calculations the Watson KEO has the important advantage that it uses an Eckart molecule-fixed axis system [92] that significantly reduce vibration-rotation coupling. Deriving a curvilinear KEO in an Eckart frame is possible but the operator one obtains is not simple [93-95].

For a description of "floppy" molecules or in general large amplitude motion geometrically defined curvilinear coordinates are better than normal coordinates. General KEOs also exist in curvilinear coordinates $[96,97]$ but they are not as simple as the Watson KEO without the $\boldsymbol{\pi}^{\boldsymbol{t}} \boldsymbol{\mu} \boldsymbol{\pi}$ term. What criteria should be considered when coordinates are chosen? (i) Coordinates (and their domains) must be chosen so that there is a one-to-one correspondence between a geometry of the molecule and a set of coordinate values. (ii) It is advantageous to choose coordinates to minimise coupling and therefore facilitate choosing good basis functions. (iii) Coordinates should also be chosen to allow one to take advantage of symmetry. (iv) It is often helpful to choose coordinates to simplify the kinetic energy operator. (v) If potential integrals are evaluated by Gauss quadrature [98] the number of points required to converge the integrals depends on the degree of the polynomial expansion of the potential (even if the potential is not explicitly expanded) and it is therefore good to choose coordinates so that the potential, when expanded in terms of polynomials of the coordinates, converges quickly.

A simple and general KEO can be derived by using the polar coordinates associated with any set of $N-1$ vectors that specifies the shape and orientation of the molecule. Chapuisat and Iung [99] were perhaps the first to adopt this sort of approach, which was also used recently in Refs. [50,100-103]. Polar coordinates have the key advantage of satisfying criteria (iv) and (i). For some molecules they will also satisfy criteria (ii) and (iii). By using the $\mathbf{J}-\mathbf{L}$ rule [104] it is possible to refine the coordinate choice to satisfy better criteria (ii) and (iii). The KEO is much simpler if one uses "orthogonal" vectors. The 
term "orthogonal" is usually taken to mean that the mass-weighted vectors are related by an orthogonal transformation to the mass-weighted nuclear position vectors. Note that the KEO in the length and angle coordinates obtained from these vectors has mixed second derivative terms.

How does one obtain the KEO in terms of the polar coordinates associated with a set of orthogonal vectors? Most methods of deriving kinetic energy operators follow one of two routes: (1) obtain the classical kinetic energy expression and then quantize it (usually using the procedure of Podolsky [105]) or (2) use the chain rule to transform a known quantum mechanical KEO (usually the space-fixed Cartesian KEO) to derive a new KEO. Chapuisat and co-workers [99-101, 106-108] have advocated the first route. Sutcliffe and Tennyson [109-113] and Handy [114] were early proponents of the second route. Mladenovic $[102,103]$ has also used the chain rule route.

Regardless of which route is taken one starts from the KEO for $N$ nuclei in space-fixed Cartesian coordinates. If the quantum mechanical operator is transformed one begins with,

$$
\begin{aligned}
\hat{T}_{\mathrm{N}} & =-\frac{1}{2} \sum_{i=0}^{N-1} \frac{1}{M_{i}}\left(\frac{\partial^{2}}{\partial X_{i}^{2}}+\frac{\partial^{2}}{\partial Y_{i}^{2}}+\frac{\partial^{2}}{\partial Z_{i}^{2}}\right) \\
& \equiv-\frac{1}{2}\left(\hat{T}_{X}+\hat{T}_{Y}+\hat{T}_{Z}\right)
\end{aligned}
$$

where $i$ is a nuclear label and $M_{i}$ a nuclear mass. For $\hat{T}_{X}$ one then does three transformations: (i) to mass-weighted coordinates $\left\{\bar{X}_{i}=M_{i}^{1 / 2} X_{i}\right\}$; (ii) to coordinates $\left\{\bar{P}_{\alpha}\right\}$ linearly related to $\left\{\bar{X}_{i}\right\}$ by an $N \times N$ orthonormal transformation; and (iii) to mass-unweighted coordinates $\left\{P_{\alpha}=\mu_{\alpha}^{-1 / 2} \bar{P}_{\alpha}\right\}$. The third step introduces arbitrary masses $\left\{\mu_{\alpha}\right\}$ :

$$
\hat{T}_{X}=-\frac{1}{2} \sum_{\alpha=0}^{N-1} \frac{1}{\mu_{\alpha}}\left(\frac{\partial^{2}}{\partial P_{\alpha}^{2}}\right)
$$

Applying an identical transformation to $\hat{T}_{Y}$ and $\hat{T}_{Z}$ (by introducing coordinates $\left\{Q_{\alpha}\right\}$ and $\left\{R_{\alpha}\right\}$ ), we obtain $N$ vectors $\left\{\mathbf{r}_{\alpha}\right\}$ (each with space-fixed Cartesian components $\left.\left(P_{\alpha}, Q_{\alpha}, R_{\alpha}\right)\right)$ that are linear combinations of the space-fixed Cartesian nuclear position vectors with coefficients that are elements of an $N \times N$ matrix

$$
\mathbf{J}=\mu^{-1 / 2} \mathbf{U M}^{1 / 2}
$$

where $\mathbf{M}$ and $\boldsymbol{\mu}$ are diagonal matrices of masses, and $\mathbf{U}$ is orthonormal. [50] $\mathbf{r}_{N-1}$ is chosen to be the position of the nuclear centre of mass. Natural coordinates for describing the shape and orientation of the molecule are: the lengths of the remaining $N-1$ vectors $r_{0}, r_{1}, \cdots r_{N-2} ; N-2$ polar angles, $\theta_{\alpha}(\alpha=1 \cdots N-2)$ between $r_{0}$ and $r_{\alpha} ; N-3$ angles $\phi_{\beta}(\beta=2 \cdots N-2)$ 
between the plane that contains $r_{0}$ and $r_{1}$ and the planes that contain $r_{0}$ and $r_{\beta}$; and three Euler angles that specify the orientation of the molecule-fixed axis system with respect to the space-fixed axes. The molecule-fixed $z$ axis is parallel to $r_{0}$ and the molecule-fixed $y$ axis is along $\mathbf{r}_{\mathbf{0}} \times \mathbf{r}_{\mathbf{1}}$. The KEO in these coordinates is given in many papers. $[100,102,115,116]$. One convenient form [117] is,

$$
T=T_{\mathrm{s}}+T_{\mathrm{br}}+T_{\text {cor }}
$$

with

$$
T_{\mathrm{br}}=T_{\mathrm{br}, \text { diag }}+T_{\mathrm{br}, \mathrm{off}}
$$

and

$$
\begin{aligned}
T_{\mathrm{s}}= & -\sum_{k=0}^{N-2} \frac{1}{2 \mu_{k}} \frac{\partial^{2}}{\partial r_{k}^{2}} \\
T_{\mathrm{br}, \text { diag }}= & {\left[B_{0}\left(r_{0}\right)+B_{1}\left(r_{1}\right)\right]\left[-\frac{1}{\sin \theta_{1}} \frac{\partial}{\partial \theta_{1}} \sin \theta_{1} \frac{\partial}{\partial \theta_{1}}+\frac{1}{\sin ^{2} \theta_{1}}\left(J_{z}-L_{z}\right)^{2}\right] } \\
& +\sum_{k=2}^{N-2}\left[B_{0}\left(r_{0}\right)+B_{k}\left(r_{k}\right)\right] l_{k}^{2} \\
& +B_{0}\left(r_{0}\right)\left[J^{2}-2\left(J_{z}-L_{z}\right)^{2}-2 J_{z}\left(L_{z}\right)+2 \sum_{k \neq k^{\prime}=2}^{N-2} l_{k z} l_{k^{\prime} z}\right] \\
T_{\mathrm{br}, \text { off }}= & B_{0}\left(r_{0}\right)\left[\left(L_{+}\right) a_{1}^{-}+\left(L_{-}\right) a_{1}^{+}+\sum_{k \neq k^{\prime}=2}^{N-2}\left(l_{k+} l_{k^{\prime}-}+l_{k-} l_{k^{\prime}+}\right)\right] \\
T_{\text {cor }}= & -B_{0}\left(r_{0}\right)\left[J_{-}\left(a_{1}^{+}+L_{+}\right)+J_{+}\left(a_{1}^{-}+L_{-}\right)\right]
\end{aligned}
$$

where

$$
\begin{gathered}
B_{i}\left(r_{i}\right)=\frac{1}{2 \mu_{i} r_{i}^{2}} \\
L_{z}=\sum_{k=2}^{N-2} l_{k z} \\
L_{-}=\sum_{k=2}^{N-2} l_{k-} \\
L_{+}=\sum_{k=2}^{N-2} l_{k+} \\
l_{i \pm}=l_{i x} \pm \imath l_{i y} \quad(i=2, \cdots, N-2) \\
J_{ \pm}=J_{x} \pm \imath J_{y}
\end{gathered}
$$




$$
a_{1}^{ \pm}= \pm \frac{\partial}{\partial \theta_{1}}-\cot \theta_{1}\left(J_{z}-L_{z}\right) .
$$

and $l_{k x}, l_{k y}, l_{k z}, l_{k}^{2}$ are the usual angular momentum operators [118]. Note that angular momentum vectors for the vectors not used to define the moleculefixed frame contribute to $L . a_{1}^{+}$and $a_{1}^{-}$act as raising and lower operators. [117] Although $a_{1}^{+}$and $a_{1}^{-}$do not commute with $l_{k \pm}$ and $J_{ \pm}$, the KEO is hermitian. The volume element associated with this KEO is $\sin \theta_{1} \sin \theta_{2} \cdots, \sin \theta_{N-2}$ $d r_{o} d r_{1} \cdots d r_{N-2} d \theta_{1} \cdots d \theta_{N-2} d \phi_{2} \cdots d \phi_{N-2}$ Note that this KEO is valid for any choice of the orthogonal vectors. Changing the definition of the vectors changes the masses that appear in the final KEO. For four-atom molecules several choices are given explicitly in Ref. [50]. There are momentum cross terms between angles but none between angles and lengths. This general "polyspherical" KEO is remarkably compact if it is written in terms of angular momentum operators. In terms of derivatives with respect to coordinates it is more lengthy.

A disadvantage of the above KEO is its lack of flexibility: one must place the molecule-fixed $z$ axis along one of the $\mathbf{r}_{\mathbf{i}}$ vectors and one must choose as vibrational coordinates polyspherical coordinates obtained from the $N-1$ $\mathbf{r}_{\mathbf{i}}$ vectors. If $r_{j}(j=2, \cdots, N-2)$ are orthogonal to $r_{0}$ and $r_{1}$ a general (and more flexible) molecule-fixed KEO can be derived, even if $r_{0}$ and $r_{1}$ are themselves not orthogonal, by attaching the molecule-fixed axis system to the plane spanned by $r_{0}$ and $r_{1}$. This is done as follows [104]: (i) Write the KEO obtained by adding Eq. (23) and its $Y$ and $Z$ counterparts, and removing the centre of mass term, as

$$
K=-\frac{1}{2 \mu_{0}} \frac{\partial^{2}}{\partial \mathbf{r}_{\mathbf{0}}^{\mathbf{2}}}-\frac{1}{2 \mu_{1}} \frac{\partial^{2}}{\partial \mathbf{r}_{\mathbf{1}}^{\mathbf{2}}}-\frac{1}{\mu_{01}} \frac{\partial}{\partial \mathbf{r}_{\mathbf{1}}} \frac{\partial}{\partial \mathbf{r}_{\mathbf{0}}}-\frac{1}{2} \sum_{i=2}^{N-2} \frac{1}{\mu_{i}} \frac{\partial}{\partial \mathbf{r}_{\mathbf{i}}} \frac{\partial}{\partial \mathbf{r}_{\mathbf{i}}}
$$

(ii) From the first three terms in Eq. (35) derive a ro-vibrational KEO for a triatomic-like system in terms of any vibrational coordinates $q_{1}, q_{2}$, and $q_{3}$ and in any molecule-fixed frame attached to the two embedding vectors $\mathbf{r}_{\mathbf{1}}$ and $\mathbf{r}_{\mathbf{0}}$;

(iii) Substitute $\mathbf{J}-\mathbf{L}$ for $\mathbf{J}$ in the triatomic-like KEO. This yields,

$$
K=K_{\text {tri }}\left(q_{1}, q_{2}, q_{3}, \mathbf{J}-\mathbf{L}\right)-\frac{1}{2} \sum_{i=2}^{N-2} \frac{1}{\mu_{i}} \frac{\partial}{\partial \mathbf{z}_{\mathbf{i}}} \frac{\partial}{\partial \mathbf{z}_{\mathbf{i}}}
$$

where the $\mathbf{z}_{\mathbf{i}}$ are the nonembedding vectors in the molecule-fixed frame. (iv) Transform from the $z_{i a}$ coordinates (Cartesian components of the $\mathbf{z}_{\mathbf{i}}$ ) to some non-Cartesian coordinates that describe the length and orientation of the $N-$ $3 \quad \mathbf{z}_{\mathbf{i}}$ vectors with respect to the molecule-fixed frame attached to $\mathbf{r}_{\mathbf{1}}$ and $\mathbf{r}_{\mathbf{0}}$. Here $\boldsymbol{l}$ is the total angular momenta of the non-embedding vectors in the molecule-fixed frame. This recipe enables one to obtain a KEO for an $N$-atom molecule from the KEOs of smaller molecules. This procedure works only because the $\mathbf{r}_{\mathbf{j}}(j=2, \cdots, N-2)$ vectors are orthogonal to the two 
embedding vectors [104]. This KEO is simple and general despite the fact that we do not require that the $z$ axis be placed along one of the $\mathbf{r}_{\mathbf{i}}$ vectors. This is important because either to reduce ro-vibrational coupling or to facilitate exploiting symmetry it is sometimes better not to have the $z$ axis along a $\mathbf{r}_{\mathbf{i}}$ vector. In Ref. [119] ideas related to those of Ref. [104] are used to derive a KEO for $\mathrm{NH}_{3}$ using hyperspherical coordinates for the $\mathrm{H}_{3}$ group. However in Ref. [119] the molecule-fixed frame is not attached to the triatomic-like group as it is in Ref. [104].

\section{Eigensolvers}

Once one has chosen a KEO (i.e., coordinates) and obtained a potential it remains to determine eigenvalues and eigenfunctions of the Hamiltonian operator. To do so one selects a basis and computes eigenvalues and eigenvectors of a finite matrix representation of the operator. In some cases it is possible to explicitly build the matrix. If the basis is too large it is necessary to extract eigenvalues using techniques that require only matrix elements of factors or terms of the full Hamiltonian. There are two classes of methods for solving the matrix eigenvalue problem: direct and iterative methods. Direct methods can be used if the basis is small enough that it is possible to store the Hamiltonian matrix in memory. For larger basis sets iterative methods are necessary.

\subsection{Direct methods}

The standard direct method uses a combination of Householder transformations and the QR algorithm [98]. To use it one must store, in memory, the matrix whose eigenvalues are desired, i.e., $N^{2}$ numbers, where $N$ is the size of the matrix, and the cost of the calculation scales as $N^{3}$. If $N$ is larger than about 50000 both the memory cost and the CPU cost are significant. Nevertheless, the Householder direct eigensolver can be used to compute energy eigenvalues. To do so one must choose good basis functions so as to minimize $N$.

\subsection{Iterative methods}

The unfavourable scaling of the Householder algorithm has prompted the investigation and development of iterative methods for calculating spectra $[45,46,50,120-131]$. An iterative method requires only the computation of matrix-vector products [132]. Hamiltonian matrix-vector products can be computed without storing the Hamiltonian matrix and without even calculating its matrix elements $[50,133,134]$. One needs only enough memory to store a few vectors with as many components as there are basis functions.

The most popular iterative methods employed to calculate spectra are variants of the Lanczos algorithm $[132,135]$, the filter diagonalisation method 
$[45,46,126,129]$, or the Davidson algorithm [136]. In the Lanczos method, eigenvalues of an $N \times N$ symmetric matrix $H$ are calculated by diagonalising an associated $M \times M$ tridiagonal matrix $T_{M}$ which is generated recursively, having dimension $M$ after $M$ iterations. To obtain $T_{M}$ one must compute $M$ matrixvector products. If $M$ is large enough, among the eigenvalues of $T_{M}$ there will be eigenvalues of the original matrix $\mathbf{H}$ [135]. There are several refined versions of the Lanczos algorithm designed to accelerate the convergence of selected eigenvalues $[137,138]$. These methods facilitate the calculation of low-lying energy levels and allow calculation of eigenvectors without re-doing matrix-vector products but they require considerably more memory than a straightforward Lanczos method.

The goal of the filter diagonalisation method is to calculate energy levels and wavefunctions in a selected (not necessarily low-lying) spectral window. One builds a matrix representation of the Hamiltonian using basis vectors $\mathbf{z}_{\mathbf{n}}=$ $f(\mathbf{H}, n) \mathbf{v}, n=1,2, \cdots L$, where $\mathbf{v}$ is a starting vector and $f(\mathbf{H}, n)$ is chosen so that the filtered basis vectors have only very small overlaps with eigenvectors of $\mathbf{H}$ whose eigenvalues are outside the window in which one wishes to calculate eigenvalues [126]. $\mathbf{v}$ and $\mathbf{H}$ are representations in some original basis of a starting ket and the Hamiltonian operator. In the filtered basis one solves a generalized eigenvalue problem to obtain energy levels. Wall and Neuhauser pointed out that if Hamiltonian and overlap matrix elements are calculated directly, eigenvalues of the Hamiltonian may be computed without computing (and storing) the $\mathbf{z}_{\mathbf{n}}$ vectors [45]. Mandelshtam and Taylor have developed a reliable Chebyshev FD algorithm [129]. Using their low-storage approach to filter-diagonalisation one is able to calculate energy levels using as little memory as one requires using a Lanczos approach. The Cullum \& Willoughby $(\mathrm{C} \& \mathrm{~W})$ Lanczos approach and the filter-diagonalisation method extract energy levels from the same Krylov space. Both may be viewed as methods for dealing with numerical error introduced as the space is generated. Formally, the space spanned by the Lanczos vectors is larger than (and includes) the space spanned by the filtered $\mathbf{z}_{\mathbf{n}}$ vectors. The C\&W Lanczos method requires about as many (in general somewhat fewer) matrix-vector products than FD and it is simpler to implement $[139,140]$.

The Davidson algorithm is a preconditioned variant of the Lanczos algorithm [136]. It works best if one has both (i) an easily invertable matrix, the preconditioner, whose eigenvalues are close to those of the full Hamiltonian matrix and (ii) a good starting vector. It is common to use a diagonal preconditioner. The Davidson algorithm favours the convergence of the lowest or (with a block version) a number of the lowest eigenvalues. It is used in the MULTIMODE program [75,76] and the improved relaxation method [141]. Davidson type approaches work well if it is possible to choose a basis that facilitates finding a good preconditioner.

If one opts for an iterative method one needs a routine for computing matrix-vector products. The matrix-vector product is the heart and soul of 
any iterative procedure. To compute matrix-vector products one should take advantage of any structure of the basis. The MCTDH basis is a direct product and this structure is very favourable. The basis used in MULTIMODE is selected from a direct product basis but has no exploitable structure. In the contracted iterative methods outlined in the next section the basis is a product of reduced dimension functions and this structure is used to evaluate matrix vector products.

In the next sections we review methods and codes in detail. We begin with Hamiltonians in polyspherical coordinates and emphasize the use of truncation-recoupling. Following that we describe variational methods based on the Watson Hamiltonian, the n-mode representation of the potential and the VSCF/VCI approach to obtain eigenvalues and eigenfunctions. Section 7 describes the MCTDH approach.

\section{Contracted iterative methods}

The simplest way to compute a eigenvalue spectrum is to use a time-independent direct-product basis and a direct eigensolver. For a triatomic molecule this is a perfectly adequate approach. To solve more difficult problems one must use a better eigensolver, use a better basis or do both. The first calculations of spectra done with iterative eigensolvers were done with direct-product bases $[50,142,143]$. Using the Lanczos algorithm it is straightforward to do calculations with millions of direct-product basis functions. The calculation can be done without representing the potential using either a potfit or a multimode expansion, regardless of whether one uses a DVR or an FBR. The sums required to evaluate matrix-vector products are done sequentially so that one obtains a eigenvalue spectrum without computing multidimensional integrals. Even the advantages of iterative methods are not enough to make it possible to compute spectra of five-atom molecules with a direct-product basis. To do so one must improve the basis. There are three ways in which the basis set can be improved: (1)only the most important basis functions can be selected from a huge direct-product basis; (2) contracted basis functions that are not simple products can be used; (3) time-dependent basis functions can be used. The MULTIMODE package of Carter, Bowman, Handy, and co-workers uses strategy (1) and very recently strategy (2). MCTDH uses strategy (3) and also (2). In this section we sketch an approach that uses strategy (2), while strategy (3) is discussed in section 7 .

Contracted basis functions are chosen to reduce the number of basis functions required to obtain converged energy levels. It has been clearly demonstrated that if they are used with a direct eignensolver they are powerful tools for computing vibrational energy levels $[6,144]$. Contracted basis functions are usually obtained by diagonalizing reduced-dimension Hamiltonian matrices. There are two popular ways to define the effective reduced-dimension Hamil- 
tonian matrices: 1) one diagonalizes blocks of the full Hamiltonian matrix in a (primitive) product basis representation; 2) one diagonalizes matrices that represent the Hamiltonian with one or more coordinates fixed. The first route leads to nondirect-product contracted basis functions [144-151]. The second route leads to basis functions that are direct-products of functions of different coordinates or groups of coordinates $[6,152]$. An example (see also Eq. (1)) should make this clearer. For a triatomic molecule, a contraction scheme of type 1 is obtained by diagonalizing (stretch) blocks $\left\langle\alpha(\theta)\left|\hat{H}\left(\theta, r_{1}, r_{2}\right)\right| \alpha(\theta)>\right.$ to get eigenfunctions $\phi_{n}^{\alpha}\left(r_{1}, r_{2}\right)$ where $\alpha(\theta)$ is a DVR bend function, $\hat{H}\left(\theta, r_{1}, r_{2}\right)$ is the full Hamiltonian operator, and $\theta, r_{1}, r_{2}$ are the bend and stretch coordinates. The contracted basis functions are then $\alpha(\theta) \phi_{n}^{\alpha}\left(r_{1}, r_{2}\right)$. A contraction scheme of type 2 is obtained by combining the eigenfunctions of $H\left(\theta_{e}, r_{1}, r_{2}\right)$, denoted $\psi\left(r_{1}, r_{2}\right)$ and eigenfunctions of $H\left(\theta, r_{1}^{e}, r_{2}^{e}\right)$, denoted $\chi(\theta)$. The contracted basis functions are $\psi\left(r_{1}, r_{2}\right) \chi(\theta) . r_{1}^{e}, r_{2}^{e}$, and $\theta_{e}$ are selected specific values of $r_{1}, r_{2}$, and $\theta$. Often equilibrium values will be good choices. Regardless of the route one takes, only some of the eigenvectors of the reduceddimension Hamiltonian matrices are retained: such methods are sometimes called diagonalization-truncation-recoupling methods [17,52].

The type 1 approach has been used extensively by Bacic, Bowman, Light, Tennyson and their coworkers $[17,52,144,145,147,148]$. Luckhaus [151] and Mladenović $[149,150]$ have recently used type 1 contractions in $6 \mathrm{D}$ calculations. Both these authors use contracted basis functions with a direct eigensolver (Householder transformations). The type 2 approach was pioneered by Carter and Handy $[6,152]$ and has been used with a direct eigensolver to compute rovibrational energy levels of four-atom molecules, e.g. $\mathrm{C}_{2} \mathrm{H}_{2}$ [153], $\mathrm{H}_{2} \mathrm{O}_{2}$ [154], $\mathrm{NH}_{3}$ (vibration only) [7].

Is it efficient to use contracted basis functions (of either type) with an iterative eigensolver? This seems like an obvious combination. Why not profit from both the smallness of the contracted basis and the reduced memory requirement of iterative eigensolvers? The combination is effective, but finding a good scheme for evaluating matrix-vector products in the contracted basis requires some thought. The ideas used for evaluating matrix-vector products in a direct-product basis exploit the special structure of the product basis to make it possible to do summations (over basis or quadrature indices) sequentially [50]. If one uses contracted basis functions at least some of this product structure is lost. The contracted basis + Lanczos combination is expected to be better than the product basis + Lanczos combination because: 1)the Lanczos vectors are smaller (reducing the memory cost) and 2)the number of required Lanczos iterations is smaller because it depends on the spectral range of the matrix which will be smaller if contracted functions are used. To realize these advantages one must have a good means of computing contracted basis matrix-vector products.

Type 1 contracted functions can be used with iterative methods $[125,146$, $155,156]$. A type 1 basis large enough to compute a desired portion of a 
eigenvalue spectrum will almost always be smaller than its type 2 counterpart. Nevertheless iterative calculations with a type 2 basis will, in most cases, be more efficient. To understand why consider the type 1 contracted matrixvector product for a triatomic. We label with $j$ functions of $q_{1}$ and $q_{2}$ obtained by diagonalising a two-dimensional Hamiltonian for each DVR point $\left(q_{3}\right)_{\gamma}$. If the two-dimensional Hamiltonian is diagonalised in a direct-product $q_{1} q_{2}$ basis and $\alpha$ and $\beta$ are DVR labels for the $q_{1}$ and $q_{2}$ DVR basis functions then the matrix of eigenvectors is the transformation matrix whose elements are $C_{\alpha \beta, j}^{\gamma}$. In a basis of functions labelled by $j$ and $\gamma$ the Hamiltonian (written in Radau, symmetrized Radau, or Jacobi coordinates) matrix elements are,

$$
\left\langle j^{\prime} \gamma^{\prime}|\hat{H}| j \gamma\right\rangle=E_{j}^{\gamma} \delta_{j^{\prime} j} \delta_{\gamma \gamma^{\prime}}+\sum_{\alpha \beta} C_{\alpha \beta, j^{\prime}}^{\gamma^{\prime}} \mu\left(\left(q_{1}\right)_{\alpha},\left(q_{2}\right)_{\beta}\right) l_{\gamma^{\prime} \gamma} C_{\alpha \beta, j}^{\gamma},
$$

where, $\mu$ is an inverse moment of inertia function and $l_{\gamma^{\prime} \gamma}$ is a DVR matrix element of an operator proportional to $\frac{\partial^{2}}{\partial \theta^{2}}+\cot \theta \frac{\partial}{\partial \theta}$. In this representation applying the Hamiltonian to a vector implicates only one non-trivial matrixvector product. When sums are evaluated sequentially the cost of the matrixvector product,

$$
\sum_{\alpha \beta} C_{\alpha \beta, j^{\prime}}^{\gamma^{\prime}} \mu\left(\left(q_{1}\right)_{\alpha},\left(q_{2}\right)_{\beta}\right) \sum_{\gamma} l_{\gamma^{\prime} \gamma} \sum_{j} C_{\alpha \beta, j}^{\gamma} v_{\gamma j},
$$

scales as $2\left(n_{j} n_{\alpha} n_{\beta} n_{\gamma}+n_{\gamma}^{2} n_{\alpha} n_{\beta}+n_{\alpha} n_{\beta} n_{\gamma}\left(n_{j}\right.\right.$ is the number of eigenfunctions of the two-dimensional Hamiltonians which are retained). If $n_{\alpha}=n_{\beta}=n_{\gamma}=n$ and $n_{j}$ is $n$ the cost of each matrix-vector product scales as $n^{4}$. In general, the cost of the type 1 contracted matrix-vector products will depend on the number of retained basis functions and is hard to estimate. However, it is clear that the number of matrix-vector products required to obtain a converged eigenvalue spectrum will be less than would be necessary if one used a product basis. This type 1 approach has several important disadvantages. (1) Many reduced-dimension problems must be solved. In the triatomic case there is only one bend angle, but for a five-atom molecule there are 5 angles and a type 1 contraction, similar to the one outlined above for triatomics, would require solving a stretch problem for millions of (multi-dimensional) DVR bend functions. (2) For five-atom molecule it would be impossible to store, if it were possible to compute, the stretch eigenfunctions for all the bend DVR points. (3) The type 1 contraction presented above derives its efficiency from the use of a DVR for the bend and exploitation of an adiabatic bend/stretch separation. Multidimensional bend DVRs that allow one to cope with KEO singularities are not known. (Note that disadvantages (1) and (2) would also cripple a type 1 approach in which a bend problem is solved for each multdimensional stretch DVR point.) (4) To evaluate the matrix-vector product in Eq. (38), the vector is transformed to a simple product representation in which it is easy to apply the KEO and then back transformed to the contracted representation. 
This inevitably entails storing a vector in the product representation. Thus despite the fact that the vector in the contracted basis is not large, one must store a large vector to evaluate matrix-vector products. Type 2 contracted functions do not have these disadvantages. We have called such functions "simply contracted" basis functions [125,157]. A type 2 contraction technique will now be described.

In orthogonal polyspherical coordinates the Hamiltonian (for $J=0$ ) of any molecule can be written as $[100,102,108]$

$$
H=T_{b e n}(\theta, r)+T_{s t r}(r)+V(\theta, r)
$$

with

$$
\begin{aligned}
T_{b e n}(\theta, r) & =\sum_{i} B_{i}(r) T_{b}^{(i)}(\theta) \\
T_{s t r}(r) & =\sum_{i} \frac{1}{2 \mu_{i}} \frac{\partial^{2}}{\partial r_{i}^{2}} .
\end{aligned}
$$

$\theta$ represents all of the bend coordinates and $r$ represents all of the stretch coordinates. The functions $B_{i}(r)$ and the operators $T_{b}^{(i)}(\theta)$ are known [100, 102].

We first construct contracted bend functions from a Hamiltonian obtained by freezing all the stretch coordinates at equilibrium (or more generally reference) values and contracted stretch functions from a Hamiltonian obtained by freezing all the bend coordinates at equilibrium (or reference) values. Products of the bend contracted functions and stretch contracted functions are our final basis functions.

The reduced-dimension Hamiltonian for the bend contraction is,

$$
H^{(b)}=T_{b e n}\left(\theta, r_{e}\right)+V\left(\theta, r_{e}\right) .
$$

Its wavefunctions are denoted by

$$
X_{b}(\theta)=\sum_{l} C_{l b} f_{l}(\theta)
$$

and the energies by $E_{b}$. The $f_{l}$ are primitive FBR or DVR bend basis functions (if there is more than one bend coordinate $l$ is a composite index) and the number of retained bend wavefunctions is denoted by $n_{b}$.

Similarly, the reduced-dimension Hamiltonian for the stretch contraction is,

$$
H^{(s)}=T_{s t r}(r)+V\left(\theta_{e}, r\right) .
$$

with the wavefunctions denoted by,

$$
Y_{s}(r)=\sum_{\alpha} D_{\alpha s} g_{\alpha}(r)
$$


and the energies by $E_{s}$. The $g_{\alpha}$ are primitive DVR stretch basis functions (if there is more than one stretch coordinate $\alpha$ is a composite index representing a multidimensional DVR function) and the number of retained stretch wavefunctions is denoted by $n_{s} . \theta_{e}$ and $r_{e}$ represent equilibrium values of (respectively) all the bend coordinates and all the stretch coordinates. The final basis is a product of the retained stretch and bend eigenfunctions

$$
|b s\rangle=\left|X_{b}\right\rangle\left|Y_{s}\right\rangle
$$

To facilitate calculation of its matrix elements in this basis we write the full Hamiltonian

$$
H=H^{(b)}+H^{(s)}+\Delta T+\Delta V
$$

where

$$
\Delta V(r, \theta)=V(r, \theta)-V\left(r_{e}, \theta\right)-V\left(r, \theta_{e}\right)
$$

and

$$
\Delta T=\sum_{i} \Delta B_{i}(r) T_{b}^{(i)}(\theta)
$$

with

$$
\Delta B_{i}(r)=B_{i}(r)-B_{i}\left(r_{e}\right) .
$$

Since $H^{(b)}$ and $H^{(s)}$ are diagonal in the product contracted basis and the diagonal matrix elements are known (they are the eigenvalues of $H^{(b)}$ and $\left.H^{(s)}\right)$ one only needs to calculate matrix elements of $\Delta T$ and $\Delta V$ in the product contracted basis. If it were possible to store the Hamiltonian matrix in the the product contracted basis $|b s\rangle$ a direct linear algebra method could be used to compute the energy levels. If this is not possible one must use an iterative method and evaluate matrix-vector products.

The iterative method we prefer is the Lanczos algorithm. We use it solve the bend problem, the stretch problem and to compute the final solutions in the basis of products of bend and stretch solutions. The Lanczos algorithm is simple and easy to use. There are no problems with spurious eigenvalues [135]. From a single set of matrix-vector products we can extract a large number of eigensolutions. It is necessary to store only a few vectors and there is no need for a good initial vector.

\subsubsection{Matrix-vector products for $\Delta T$}

Due to the product structure of Eq. (48) it is best to store the bend and stretch matrices separately. The matrix-vector product for $\Delta T$ can be performed sequentially without summing over $n_{b}$ bend functions and $n_{s}$ stretch functions simultaneously. Note that it not necessary to store any vectors in the primitive basis. 
Specifically, the $\Delta T$ matrix-vector product can be calculated

$$
u_{b^{\prime} s^{\prime}}^{\prime}=\sum_{i, s}\left\langle s^{\prime}\left|\Delta B_{i}\right| s\right\rangle \sum_{b}\left\langle b^{\prime}\left|T_{b}^{(i)}\right| b\right\rangle u_{b s}
$$

The $\Delta B_{i}$ and $T_{b}^{(i)}$ matrices are both small and are stored. The $\Delta B_{i}$ matrix elements are computed from

$$
\left\langle s^{\prime}\left|\Delta B_{i}\right| s\right\rangle=\sum_{\alpha} D_{\alpha s^{\prime}} \Delta B_{i}\left(r_{\alpha}\right) D_{\alpha s} .
$$

The $T_{b}^{(i)}$ matrix elements are computed from

$$
\left\langle b^{\prime}\left|T_{b}^{(i)}\right| b\right\rangle=\sum_{l, l^{\prime}} C_{l^{\prime} b^{\prime}}\left\langle f_{l}\left|T_{b}^{(i)}\right| f_{l^{\prime}}\right\rangle C_{l b} .
$$

Assuming that $n_{b}>n_{s}$ (which almost always holds) the memory cost of the $\Delta T$ matrix-vector product is $n_{b}^{2}$ and the CPU cost is $n_{b}^{2} n_{s}$. The memory and CPU costs of the $\Delta V$ matrix-vector product are much higher [157].

\subsection{Matrix-vector products for $\Delta V$}

In this subsection we present a method for calculating the $\Delta V$ matrix-vector product [157]. If one uses an FBR primitive bend basis a matrix element of $\Delta V$ in the product contracted basis is,

$$
\begin{aligned}
\left\langle b^{\prime} s^{\prime}|\Delta V(\theta, r)| b s\right\rangle & =\sum_{\substack{l^{\prime} l \\
\alpha}} C_{l^{\prime} b^{\prime}} C_{l b} D_{\alpha s^{\prime}} D_{\alpha s}\left\langle l^{\prime} \alpha|\Delta V(\theta, r)| l \alpha\right\rangle \\
& =\sum_{\substack{l^{\prime} l \\
\alpha \beta}} C_{l^{\prime} b^{\prime}} C_{l b} D_{\alpha s^{\prime}} D_{\alpha s} T_{l^{\prime} \beta} T_{l \beta} \Delta V_{\beta \alpha}
\end{aligned}
$$

where

$$
\begin{aligned}
T_{l \beta} & =\sqrt{w_{\beta}} f_{l}\left(\theta_{\beta}\right) \\
\Delta V_{\beta \alpha} & =\Delta V\left(\theta_{\beta}, r_{\alpha}\right) .
\end{aligned}
$$

$\left(\theta_{\beta}, w_{\beta}\right)$ are quadrature points and weights for the angles. Note that if there is more than one bend coordinate $l$ and $\beta$ are composite indices and if there is more than one stretch coordinate $\alpha$ is a composite index. To do matrix-vector products of $\Delta V$ efficiently there is no need to represent it either as a sum of products or in multimode form. It is sometimes advantageous to rewrite Eq. (53) as

$$
\left\langle b^{\prime} s^{\prime}|\Delta V(\theta, r)| b s\right\rangle=\sum_{\alpha \beta} \tilde{C}_{\beta^{\prime} b^{\prime}} \tilde{C}_{\beta b} D_{\alpha s^{\prime}} D_{\alpha s} \Delta V_{\beta \alpha}
$$


where

$$
\tilde{C}_{\beta b}=\sum_{l} C_{l b} T_{l \beta} .
$$

A potential matrix element in the product contracted basis is rewritten

$$
\begin{aligned}
\left\langle b^{\prime} s^{\prime}|\Delta V(\theta, r)| b s\right\rangle & =\sum_{\alpha \beta} \tilde{C}_{\beta^{\prime} b^{\prime}} \tilde{C}_{\beta b} D_{\alpha s^{\prime}} D_{\alpha s} \Delta V_{\beta \alpha} \\
& =\sum_{\alpha} F_{b^{\prime} b, \alpha} D_{\alpha s^{\prime}} D_{\alpha s}
\end{aligned}
$$

where we have introduced an $\mathbf{F}$ matrix defined by,

$$
F_{b^{\prime}, b, \alpha}=<b^{\prime} \mid \Delta V\left(\theta, r_{\alpha} \mid b>=\sum_{\beta} \tilde{C}_{\beta b^{\prime}} \tilde{C}_{\beta b} \Delta V_{\beta \alpha} .\right.
$$

To use this method one calculates and stores all the $F_{b^{\prime} b, \alpha}$. One then does the $\Delta V$ matrix-vector product,

$$
u_{b^{\prime} s^{\prime}}^{\prime}=\sum_{b s}\left\langle b^{\prime} s^{\prime}|\Delta V| b s\right\rangle u_{b s} .
$$

This is most efficiently done in three steps:

$$
\begin{aligned}
u_{b \alpha}^{(1)} & =\sum_{s} D_{\alpha s} u_{b s} \\
u_{b^{\prime} \alpha}^{(2)} & =\sum_{b} F_{b^{\prime} b \alpha} u_{b \alpha}^{(1)} \\
u_{b^{\prime} s^{\prime}}^{\prime} & =\sum_{\alpha} D_{\alpha s^{\prime}} u_{b^{\prime} \alpha}^{(2)}
\end{aligned}
$$

Full dimensional integrals are never computed. The CPU cost of the matrixvector product in Eq. (60) is $n_{\alpha}\left(n_{b}^{2}+2 n_{b} n_{s}\right)$. Assuming $n_{b}>n_{s}$ the CPU cost scales as $n_{\alpha} n_{b}^{2}$. The calculation of $\mathbf{F}$ can be easily parallelized. For example, one can allocate the stretch DVR $(\alpha)$ points to different computers or processors and allow each to calculate a block of $\mathbf{F}$ for the allocated $\alpha$ points.

In previous approaches for using contracted basis functions in conjunction with iterative eigensolvers one evaluated a matrix-vector product by transforming from the contracted basis to a direct product basis, applying an operator, and then transforming back. This has the disadvantage that it requires storing a vector with as many components as there are product basis funtions. To solve the Schroedinger equation using such an approach one would need to store a vector with $N^{f}$ components, where $N$ is a representative number of basis functions (or DVR points) for a single cooridnate. If $N \sim 20$ and $f=9$ this requires more than 4000 GB! Using the approach outlined above one needs 
much less memory. It is necessary only to store $\mathbf{F}$ or $n_{b}^{2} n_{\alpha} / 2$ numbers. For the methane calculation reported in section 8.1 this requires less than 4 GB. The cost of the most expensive matrix-vector product scales as $n_{\alpha} n_{b}^{2}$, which is obviously smaller if $n_{b}$ is small, i.e., if the bend contraction works wells. Typically the matrix-vector products are much less costly than direct-product matrix-vector products would be if it were possible to store the direct-product vector.

These ideas were first applied to compute energy levels of $\mathrm{H}_{2} \mathrm{O}_{2}$ for which it is possible to do Lanczos calculations with a product basis [157] and it was demonstrated that for this problem the method is efficient. Later the same approach was used for methane for which a direct-product calculation is impossible. [158] For this molecule the bend calculation is the hardest part [54]. Later some $J>0$ levels were also computed [117]. Similar ideas based on contraction and truncation were used to compute high-lying vibrational levels of the vinylidene/acetylene system [23-25]; Ref. [24] used methods most closely related to those described in this section. Yu has studied several molecules using similar ideas $[159,160]$. He contracts only the bend part and not the stretch part, but the greatest benefit is derived from the bend contraction. Other scientists have modified and applied the approach of Ref. [157] [161, $162]$.

\section{VSCF/VCI with the Watson Hamiltonian}

The vibrational SCF approach to obtain eigenfunctions and eigenvalues of the Watson Hamiltonian begins with a Hartree product for the total wavefunction

$$
\Psi_{n_{1} \ldots n_{N}}^{V S C F}\left(Q_{1}, \ldots Q_{N}\right)=\prod_{i=1}^{N} \phi_{n_{i}}^{(i)}\left(Q_{i}\right)
$$

where the modal wavefunctions $\phi_{n_{i}}^{(i)}\left(Q_{i}\right)$, subject to the usual normalization constraint, are solutions of the coupled equations

$$
\left[T_{l}+<\prod_{i \neq l}^{N} \phi_{n_{i}}^{(i)}\left(Q_{i}\right)\left|T_{c}+V\right| \prod_{i \neq l}^{N} \phi_{n_{i}}^{(i)}\left(Q_{i}\right)>-\epsilon_{n_{l}}^{(l)}\right] \phi_{n_{l}}^{(l)}\left(Q_{l}\right)=0
$$

where

$$
T_{l} \equiv-\frac{1}{2} \sum_{k} \frac{\partial^{2}}{\partial Q_{k}^{2}}, T_{c} \equiv \frac{1}{2} \sum_{\alpha, \beta} \hat{\pi}_{\alpha} \mu_{\alpha \beta} \hat{\pi}_{\beta}-\frac{1}{8} \sum_{\alpha} \mu_{\alpha \alpha}
$$

and the integration is over the coordinates of N-1 modes. These equations are solved in the usual iterative fashion.

After achieving convergence each VSCF effective Hamiltonian has a set of virtual eigenfunctions which can be used in a standard "CI" procedure to obtain eigenvalues and eigenfunctions of the Hamiltonian. This approach is 
denoted VSCF/VCI. (The non-orthogonal basis of VSCF states can also be used in a CI $[10,11]$; however, since this is not routinely done now we do not describe this any further.)

It is immediately clear that matrix elements of the Hamiltonian, both the potential and $T_{c}$ for molecules larger than tetraatomics are not feasible for general potentials. The n-mode representation of the potential, and for $T_{c}$ are used to extend the approach to larger molecules. Current typical computational power limits $\mathrm{n}$ to a maximum of 6 . For $T_{c}$ a relatively small value of $\mathrm{n}$ is sufficient, e.g., $\mathrm{n}=2$ or 3 for most applications since this term is generally small compared to $V$ and also more nearly separable than $V$.

The implementation of the n-mode representation of the potential together with the rigorous Watson Hamiltonian was first done in the code MULTMODE $[57,76,77]$. There now exist several other codes that use the n-mode representation of the potential to obtain eigenfunctions and eigenvalues of the rigorous Watson Hamiltonian using VSCF/VCI methods [62,78].

The choice of $\mathrm{n}$ for the $\mathrm{nMR}$ of $\mathrm{V}$ also has consequences for selecting the CI excitation space. This can be seen by considering a potential matrix element for a specific p-mode potential, denoted compactly as $V^{(p)}\left(\left\{Q_{p}\right)\right\}$ for a tetraatomic where $\mathrm{N}=6$ and where $\mathrm{p}$ is less than $\mathrm{N}$ of course. The matrix element is of the reduced form

$$
<n_{1}^{\prime} n_{2}^{\prime} n_{3}^{\prime} n_{4}^{\prime} n_{5}^{\prime} n_{6}^{\prime}\left|V^{(p)}\left(\left\{\mathbf{Q}_{p}\right\}\right)\right| n_{1} n_{2} n_{3} n_{4} n_{5} n_{6}>=\delta_{n_{i}^{\prime}, n_{i}} \ldots \delta_{n_{j}^{\prime}, n_{j}} V_{\mathbf{n}_{p}^{\prime}, \mathbf{n}_{p}}^{(p)}
$$

where there are 6-p Kronecker delta functions. Clearly then for an nMR of V the matrix $\mathbf{V}$ is built from a series of p-mode terms ranging from a very sparse 2-mode component to the increasingly less sparse components as $\mathrm{p}$ increases to the value of $n$.

A detailed scheme based on the structure of the nMR of $V$ has been adopted in MULTIMODE [75]. Other schemes have also been suggested, in particular we note the approach of Rauhut [81] where the reference VSCF state from which a virtual space is selected is chosen to be "close" to energies of the states of interest. In the approach taken by Carter, Bowman and Handy and co-workers in MULTIMODE, the VSCF reference state is typically the ground vibrational state, although this is not a limitation of the code.

A truncation-recoupling contraction scheme considering the $\mathrm{nMR}$ of $\mathrm{V}$ has very recently been implemented in MULTIMODE by Carter, Handy and Bowman $[163,164]$. In this approach the potential is given by three different nMRs as follows:

$$
V\left(\mathbf{Q}_{\mathbf{s}}, \mathbf{Q}_{\mathbf{b}}\right)=V^{n_{s} M R}\left(\mathbf{Q}_{\mathbf{s}}\right)+V^{n_{b} M R}\left(\mathbf{Q}_{\mathbf{b}}\right)+V^{n_{s b} M R}\left(\mathbf{Q}_{\mathbf{s}}, \mathbf{Q}_{\mathbf{b}}\right)
$$

where $\mathbf{Q}_{\mathbf{s}}$ denotes system modes, and $\mathbf{Q}_{\mathbf{b}}$ denotes bath modes. We note that partitioning modes into groups was suggested by Scribano and Benoit for the $2 \mathrm{MR}$ of the potential [36]. 
Table 1: $J=0$ energies and rotation constants from $J=1$ energies $\left(\mathrm{cm}^{-1}\right)$ for the first fifteen vibrational states of $\mathrm{HO}_{2}$. Benchmark results are from an independent calculation done in Jacobi coordinates.

\begin{tabular}{ccccc|cccc}
\hline \hline & \multicolumn{4}{c|}{ MULTIMODE } & \multicolumn{4}{c}{ Benchmark } \\
No. & $\mathrm{J}=0$ & $\mathrm{~A}$ & $\mathrm{~B}$ & $\mathrm{C}$ & $\mathrm{J}=0$ & $\mathrm{~A}$ & $\mathrm{~B}$ & $\mathrm{C}$ \\
\hline 1 & 1065.49 & 20.46 & 1.12 & 1.06 & 1065.49 & 20.46 & 1.12 & 1.06 \\
2 & 1296.38 & 20.20 & 1.09 & 1.03 & 1296.38 & 20.20 & 1.10 & 1.04 \\
3 & 2091.12 & 21.25 & 1.12 & 1.06 & 2091.12 & 21.24 & 1.13 & 1.06 \\
4 & 2359.53 & 19.94 & 1.07 & 1.01 & 2359.54 & 19.94 & 1.07 & 1.01 \\
5 & 2516.68 & 20.96 & 1.09 & 1.04 & 2516.68 & 20.95 & 1.11 & 1.04 \\
6 & 3081.02 & 22.10 & 1.12 & 1.06 & 3081.02 & 22.08 & 1.14 & 1.06 \\
7 & 3333.68 & 19.71 & 1.05 & 1.00 & 3333.69 & 19.70 & 1.05 & 0.99 \\
8 & 3387.01 & 19.67 & 1.13 & 1.06 & 3387.01 & 19.66 & 1.13 & 1.06 \\
9 & 3589.80 & 20.63 & 1.07 & 1.02 & 3589.80 & 20.61 & 1.09 & 1.02 \\
10 & 3725.78 & 21.75 & 1.09 & 1.03 & 3725.77 & 21.73 & 1.12 & 1.04 \\
11 & 4041.28 & 23.03 & 1.1 & 1.06 & 4041.29 & 23.00 & 1.14 & 1.06 \\
12 & 4368.56 & 19.49 & 1.02 & 0.97 & 4368.57 & 19.49 & 1.03 & 0.97 \\
13 & 4421.44 & 20.27 & 1.06 & 1.00 & 4421.46 & 20.25 & 1.07 & 1.00 \\
14 & 4615.59 & 19.54 & 1.09 & 1.03 & 4615.59 & 19.53 & 1.10 & 1.03 \\
15 & 4688.32 & 21.36 & 1.08 & 1.02 & 4688.34 & 21.34 & 1.10 & 1.02 \\
\hline
\end{tabular}

Assuming the same decomposition holds for all other terms in the exact Hamiltonian the full (assumed real) Hamiltonian matrix is given by

$$
\mathbf{H}=\mathbf{H}_{\mathrm{s}}^{\mathrm{n}_{\mathrm{s}} \mathrm{MR}}+\mathbf{H}_{\mathrm{b}}^{\mathrm{n}_{\mathrm{b}} \mathrm{MR}}+\mathbf{H}_{\mathrm{sb}}^{\mathrm{n}_{\mathrm{sb}} \mathrm{MR}},
$$

where the notation should be clear $\left(\mathbf{H}_{\mathbf{s}}^{\mathbf{n}_{\mathbf{s}} \mathbf{M R}}\right.$ and $\mathbf{H}_{\mathbf{b}}^{\mathbf{n}_{\mathbf{b}} \mathbf{M R}}$ are direct-product matrices of the same order)and where we note that the dimension of $\mathbf{H}$ could be quite large. Proceeding as usual with a contraction scheme (see the Introduction and also Section 5) the matrices $\mathbf{H}_{\mathbf{s}}^{\mathbf{n}_{\mathbf{s}} \mathbf{M R}}$ and $\mathbf{H}_{\mathbf{b}}^{\mathbf{n}_{\mathbf{b}} \mathbf{M R}}$ are separately diagonalized and a truncated basis of the multidimensional eigenfunctions is used in the final step to obtain eigenfunctions of the full Hamiltonian. matrix. In this multidimensional basis the $\mathrm{H}$-matrix is given by

$$
<s^{\prime} b^{\prime}|H| s b>=\varepsilon_{s} \delta_{b^{\prime} b}+\varepsilon_{b} \delta_{s^{\prime} s}+<s^{\prime} b^{\prime}\left|H_{s b}\right| s b>
$$

where $\varepsilon_{s}$ and $\varepsilon_{b}$ are the eigenvalues of $\mathbf{H}_{\mathbf{s}}^{\mathbf{n}_{\mathbf{s}} \mathbf{M R}}$ and $\mathbf{H}_{\mathbf{b}}^{\mathbf{n}_{\mathbf{b}} \mathbf{M R}}$, respectively.

As usual for this scheme to be effective the dimension of the H-matrix in the multidimensional basis must be much smaller than the dimension of the underlying basis used to obtain the system and bath eigenfunctions. A further, hoped-for efficiency comes from using different levels of mode coupling in the potentials describing the "system" and the "bath". It is also possible to treat the off-diagonal $\left\langle s^{\prime} b^{\prime}\left|H_{s b}\right| s b>\right.$ terms if they are small with PT. In addition, off-diagonal terms can also be ignored entirely. The approach ignoring the 
off-diagonal terms but where the number of modes in the system is changed has already been investigate recently for the water dimer and trimer [163,164].

Before we conclude this subsection we note that MULTIMODE can do "exact" calculations for $J>0$, the quotes are added because the nMR is an approximation, albeit a highly controlled one. This capability was first demonstrated for $\mathrm{HO}_{2}$ and $\mathrm{H}_{2} \mathrm{O}$ [82], for which a $3 \mathrm{MR}$ is exact. A small selection of results of those test calculations for are shown in Tab. 1 where excellent agreement with benchmark results for $\mathrm{HO}_{2}[165]$ is seen.

\section{$7 \mathrm{MCTDH}$}

\section{$7.1 \quad$ Introduction}

Multiconfiguration Time-Dependent Hartree (MCTDH) is an algorithm for propagating wavefunctions of several dimensions. The MCTDH algorithm was introduced in 1990 by Meyer, Manthe, and Cederbaum [166]. A first comprehensive description of the method - together with the first non-trivial application (photodissociation of $\mathrm{NOCl}$ ) - appeared two years later [167]. The basic theory of MCTDH has been discussed in great detail in two review articles $[71,168]$. Hence in the following only a brief overview of MCTDH theory is given, highlighting the features that give the method its power and flexibility.

In contrast to the other methods discussed in this review article, MCTDH is a time-dependent method. As such it is well suited to study problems where the essential physics is finished after a rather short propagation time, e. g. photodissociation or scattering processes. Moreover, the time-dependent approach has several advantages. The time-dependent Schrödinger equation poses an initial value rather than an eigenvalue problem. This makes it easier to concentrate on computing only desired quantities, a feature which is the more desirable the larger the system. However, turning to spectroscopy of bound systems, where the vibrations 'live' forever and the autocorrelation functions never vanish, it is at the first glance not obvious that a timedependent method can compete with time-independent ones. Here, filterdiagonalization (FD) $[49,126,169]$ (see also Sec. 7.3 ) helps, as it allows to compute accurate eigenvalues while requiring reasonably short propagation times.

Finally, a recent development of MCTDH, improved relaxation [141], allows to solve the time-independent Schrödinger equation, i. e. to compute eigenvalues and eigenstates, while using all the MCTDH machinery. However, improved relaxation fails to converge if the density of states is too high. For larger system, more than four atoms, say, improved relaxation can determine only low lying states. It is an excellent method for obtaining ground states. 


\subsection{MCTDH Theory}

\subsubsection{Wavefunction Ansatz and Equations of Motion}

The basis of the MCTDH method is the use of the following wavefunction ansatz to solve the time-dependent Schrödinger equation for a physical system with $f$ degrees of freedom (DOFs) described by coordinates $q_{1}, \ldots q_{f}$ :

$$
\begin{aligned}
\Psi\left(q_{1}, \ldots q_{f}, t\right) & =\Psi\left(Q_{1}, \ldots Q_{p}, t\right) \\
& =\sum_{j_{1}=1}^{n_{1}} \cdots \sum_{j_{p}=1}^{n_{p}} A_{j_{1} \ldots j_{p}}(t) \varphi_{j_{1}}^{(1)}\left(Q_{1}, t\right) \ldots \varphi_{j_{p}}^{(p)}\left(Q_{p}, t\right) \\
& =\sum_{J} A_{J} \Phi_{J} .
\end{aligned}
$$

Eq. (68) is a direct product expansion of $p$ sets of orthonormal time-dependent basis functions $\left\{\varphi^{(\kappa)}\right\}$, known as single-particle functions (SPFs). The coordinate for each set of $n_{\kappa}$ functions is a composite or "logical" coordinate of one or more "physical" coordinates

$$
Q_{\kappa}=\left(q_{a}, q_{b}, \ldots\right) .
$$

Thus the basis functions are $d$-dimensional, where $d$ is the number of system coordinates that have been combined together and treated as one "particle". (Typically $d=1-4$ ). The third line of Eq. (68) defines the composite index $J=\left(j_{1} \ldots j_{p}\right)$ and the Hartree product $\Phi_{J}$. The ansatz looks similar to the standard wavepacket expansion $[43,170,171]$, except that the SPFs provide a time-dependent basis set.

The SPFs are numerical functions and are represented in a standard way through a primitive basis

$$
\varphi_{j_{\kappa}}^{(\kappa)}\left(Q_{\kappa}, t\right)=\sum_{\ell_{1}=1}^{N_{1, \kappa}} \cdots \sum_{\ell_{d}=1}^{N_{d, \kappa}} c_{j_{\kappa} \ell_{1} \cdots \ell_{d}}^{(\kappa)}(t) \chi_{\ell_{1}}^{(1, \kappa)}\left(q_{1, \kappa}\right) \cdots \chi_{\ell_{d}}^{(d, \kappa)}\left(q_{d, \kappa}\right) .
$$

As primitive basis $\{\chi\}$ we usually employ a DVR.

The ansatz Eq. (68) is not unique as both the coefficients and the SPFs are time-dependent. A modification of the wavefunction accomplished by changing the coefficients may alternatively be achieved by changing the SPFs. Hence one needs to introduce constraints which do not narrow the variational space but uniquely distribute time changes of the wavefunction to coefficients and SPFs. The simplest and most often used constraint reads

$$
<\varphi_{j}^{(\kappa)} \mid \dot{\varphi}_{\ell}^{(\kappa)}>=0 .
$$

This constraint has a vivid interpretation. Any variation that can be done by changing the $A$-coefficients will be done by the $A$-coefficients. Only when the 
wavefunction moves into a direction which is not covered by the current SPF basis, the SPFs will change.

Using the ansatz Eq. (68) and the constraint Eq. (71), one obtains as variational solution to the time-dependent Schrödinger equation a coupled set of equations, one for the expansion coefficients:

$$
i \dot{\mathbf{A}}=\mathcal{K} \mathbf{A},
$$

and one for each set of SPFs

$$
i \dot{\boldsymbol{\varphi}}^{(\kappa)}=\left(1-P^{(\kappa)}\right)\left(\boldsymbol{\rho}^{(\kappa)}\right)^{-1} \mathcal{H}^{(\kappa)} \boldsymbol{\varphi}^{(\kappa)} .
$$

A matrix notation has been used with the $A$-coefficients and SPFs written as vectors, i. e. $\varphi^{(\kappa)}=\left(\varphi_{1}^{(\kappa)}, \cdots, \varphi_{n_{\kappa}}^{(\kappa)}\right)^{T}$.

The matrix $\mathcal{K}$ is the Hamiltonian operator represented in the basis of Hartree products

$$
\mathcal{K}_{J L}=\left\langle\Phi_{J}|H| \Phi_{L}\right\rangle
$$

Thus Eq. (72) has a similar form as the equations of motion for standard wavepacket propagation. The difference is that the Hamiltonian matrix is time-dependent due to the time-dependence of the SPFs.

The equations of motion for the SPFs contain three new entities. The first is the projector onto the space spanned by the SPFs

$$
P^{(\kappa)}=\sum_{j}\left|\varphi_{j}^{(\kappa)}\right\rangle\left\langle\varphi_{j}^{(\kappa)}\right| \quad .
$$

The operator $\left(1-P^{(\kappa)}\right)$ ensures that the time-derivative of the SPFs is orthogonal to the space spanned by the functions.

For the other two new entities it is useful to introduce the single-hole function, $\Psi_{a}^{(\kappa)}$, which is the wavefunction associated with the $j$-th SPF of the $\kappa$-th particle. As the total wavefunction lies in the space spanned by the SPFs one can make use of the completeness relation and write

$$
\Psi=\sum_{a}\left|\varphi_{a}^{(\kappa)}\right\rangle\left\langle\varphi_{a}^{(\kappa)} \mid \Psi\right\rangle=\sum_{a} \varphi_{a}^{(\kappa)} \Psi_{a}^{(\kappa)}
$$

Using this notation, the mean-field operator matrix, $\mathcal{H}^{(\kappa)}$ can be written as

$$
\mathcal{H}_{a b}^{(\kappa)}=\left\langle\Psi_{a}^{(\kappa)}|H| \Psi_{b}^{(\kappa)}\right\rangle .
$$

The integration in the brackets is over all particles except $\kappa$. This operator on the $\kappa$-th particle correlates the motion between the different sets of SPFs.

Finally, the density matrix $\boldsymbol{\rho}^{(\kappa)}$ is

$$
\rho_{a b}^{(\kappa)}=\left\langle\Psi_{a}^{(\kappa)} \mid \Psi_{b}^{(\kappa)}\right\rangle .
$$


The density matrices, which enter the equations of motion for the SPFs, Eq. (73), can be used to provide a useful measure of the quality of the calculation. In an analogous way to the use of density matrices in electronic structure theory, the eigenfunctions of this matrix are termed natural orbitals and the eigenvalues provide populations for these functions. The lower the population, the less important the function. The space spanned by the natural orbitals is equivalent to that of the original SPFs, and if the population of the highest natural orbital is such that the function is effectively not required for an accurate description of the evolving wavepacket, the MCTDH wavefunction is converged.

\subsubsection{Efficiency and Memory Requirements}

Standard wavepacket dynamics uses a wavefunction ansatz like that of Eq. (68), except with a set of time-independent basis functions for each DOF rather than a set of time-dependent functions for each particle. While the number of basis functions may vary for each DOF, if $N$ is representative of this number, then the wavefunction is represented by $N^{f}$ expansion coefficients. This is the basis of the exponential increase of computer resources with system size that plagues wavepacket dynamics. Assuming $N=32$, a 4-dimensional system using double-precision complex arithmetic requires $16 \mathrm{MB}$ of memory just to store one wavefunction, while a 6 -dimensional system requires $16 \mathrm{~GB}$, and a 8-dimensional one $16 \mathrm{~TB}$. Clearly this scaling severely limits the size of system treatable by these methods.

An MCTDH wavefunction, on the other hand, is very compact, since the number of SPFs per particle is usually much smaller than the number of grid points used to represent a SPF. The number of data-points needed to store one MCTDH wavefunction is

$$
\text { memory } \sim n^{p}+p n N^{d},
$$

where $n$ and $N$ are characteristic of the number of SPFs and grid points per DOF, respectively. The first term is the number of $A$-coefficients, and the second accounts for representing of the SPFs on the primitive grid. Of course, the number of SPFs, $n$, necessary for convergence increases with $d$, the degree of combination, but this increase is slow, often almost linear in $d$. To give an example we assume an 8-dimensional wavefunction with $p=4, d=2, n=$ $16, N=32$. This wavefunction takes only $2 \mathrm{MB}$.

The reduction of memory requirement is paralleled by a reduction of numerical effort. The effort of the standard method scales like $N^{f+1}$ while the MCTDH effort can be estimated by a sum of two terms:

$$
\text { effort } \sim c_{1} p^{2} n^{p+1}+c_{2} p n N^{2 d},
$$

where $c_{1}$ and $c_{2}$ are constants of proportionality. The first term is due to building the mean-field matrices and calculating the time-derivative of the $A$ - 
coefficients. The second term is due to the operation of the Hamiltonian on the SPFs.

Thus if $p$ is large, the effort for the algorithm is dominated by the building of the mean-field matrices. If $p$ is small and $d$ large, the second-term, the one for the propagation of the SPFs, dominates due to the high dimensionality of the functions. Again we see the trade between the effort required for the coefficients and the SPFs which can be altered by suitably combining DOFs together into particles, balancing the reduced effort due to low $p$ with increased effort due to increasing $N^{d}$.

The evaluation of the Hamiltonian matrix elements $\mathcal{K}_{J L}$ and the building of the mean fields requires $f$ and $(f-d)$ dimensional integrals to be done at every time step. If one would do these integrals by quadrature over the primitive grid, MCTDH would not be able to compete with other methods. A fast method for evaluating the multi-dimensional integrals is essential for the success of MCTDH. One such fast algorithm is the CDVR method developed by U. Manthe [72]. However, we prefer to make use of a product representation of the Hamiltonian as already discussed in Sec. 2.2. The estimate of the effort, Eq. (80), was derived while assuming a Hamiltonian in product form. The constants of proportionality, $c_{1}$ and $c_{2}$, increase linearly with $s$, the number of terms of the product expansion Eq. (3).

Finally we note that one usually employs the constant mean-field (CMF) integration scheme $[71,172]$ when solving the MCTDH equations of motion. CMF takes advantage of the fact that the mean fields vary less rapidly than the SPFs or the $A$-coefficients and hence may be built using a coarser timegrid than the one used for propagating the $A$-coefficients and SPFs. The CMF integration scheme usually speeds up a calculation by factors between 3 and 12 as compared to solving the whole coupled set of equations with a standard all-purpose integrator.

\subsection{Fourier Spectra and Filter Diagonalization}

A photo-absorption spectrum, $\sigma(\omega)$, can be calculated from the Fourier transform of an autocorrelation function

$$
\sigma(\omega) \propto \omega \operatorname{Re} \int_{0}^{T} d t g(t) C(t) e^{i\left(E_{0}+\omega\right) t},
$$

where $E_{0}$ denotes the ground-state energy and where the autocorrelation function is defined as

$$
C(t)=\langle\Psi(0) \mid \Psi(t)\rangle \quad,
$$

A derivation is given e. g. in Ref. [173]. See also Refs. [174,175] for a formula which provides absolute intensities. The initial state to be propagated, $\Psi(0)$, is the ground state operated by the transition operator, $\Psi(0)=\hat{\mu} \Psi_{G S}$. The damping function $g(t)$ is introduced to reduce spurious effects (Gibbs phenomenon) introduced by the finite propagation time $T$. A convenient choice 
is

$$
g(t)=\cos \left(\frac{\pi t}{2 T}\right) \Theta\left(1-\frac{|t|}{T}\right),
$$

where $\Theta$ denotes the Heaviside step function. The damping function smoothly forces the integrand to be 0 at $T$. The effect of the filter $g(t)$ is that the exact spectrum is convoluted with $\tilde{g}(\omega)$, the Fourier transform of $g(t)$.

$$
\tilde{g}(\omega)=\frac{4 \pi T \cos (\omega T)}{(\pi-2 \omega T)(\pi+2 \omega T)} .
$$

The full width at half maximum of this function is $27.3 \mathrm{~cm}^{-1} \mathrm{ps}$. Hence one has to propagate for $27 \mathrm{ps}$ to achieve a resolution of $1 \mathrm{~cm}^{-1}$. Although one may save a factor of 2 by using the "T/2 trick" [71, 176, 177], such a long propagation is in general not realistic. Fourier spectra mostly show a resolution between 10 and $100 \mathrm{~cm}^{-1}$. There are several applications for which such a resolution is sufficient, e. g. when spectra are congested and only the envelope but not the individual lines are of interest [178-183].

To improve the situation, Filter Diagonalization (FD) was proposed by Neuhauser [126]. This hybrid approach promises to combine the advantages of wavepacket propagation with obtaining accurate eigenvalues by diagonalization. The idea is to build a suitable small basis to represent the Hamiltonian in a particular energy range. To do this a wavepacket is propagated and Fourier transformed on an set of, typically equidistant, energies $E_{1}, \ldots, E_{L}$. The Hamiltonian is then diagonalized in this set of filtered states. In practice one does not Fourier transform the wavepacket (which would be very elaborate), but works with the autocorrelation function only $[49,126]$. FD is hence a more clever way to analyze the autocorrelation function as compared to just Fourier transform it.

One problem is that FD can produce spurious eigenvalues. This can be a serious problem if there are errors in the propagation, something which cannot be avoided in MCTDH. A way of removing the spurious eigenvalues is to solve the eigenvalue problem using different filtering functions and different variational principles. Eigenvalues that appear in all the calculations are real, the others spurious [169].

This MCTDH/FD scheme works well for small systems like $\mathrm{CO}_{2}$ [169] or $\mathrm{HO}_{2}$ [184]. For large systems, however, one is plagued with a large number of spurious eigenvalues and it is difficult to identify and remove them. For large systems one hence adopts a different strategy. One no longer tries to generate the full spectrum by one calculation, but concentrates on the computation of the eigenenergy of a selected state. An initial state is built which is as close to the sought state as possible. The FD spectrum of such a state will show one strong line, the sought eigenstate, and several small lines, real or spurious, of no further interest. This approach, which may be called filtering by the initial state, was successfully applied in Refs. $[175,185]$. 


\subsection{Improved Relaxation}

Improved relaxation $[141,168]$ is essentially a multiconfiguration self-consistent field (MCSCF) approach. Initially this method was implemented as a modification of the well-known relaxation method [186], which is based on propagation in negative imaginary time. Using the CMF propagation scheme, the propagation of the coefficients is decoupled from the propagation of the SPFs. The modification consisted in determining the $A$-vector no longer by relaxation but by diagonalization of the Hamiltonian matrix $\mathcal{K}$. Hence the name: improved relaxation.

To derive the working equations of the improved relaxation algorithm in a rigorous way, we employ the variational principle of time-independent quantum mechanics

$$
\begin{aligned}
\delta\left\{\langle\Psi|H| \Psi\rangle-E\left(\sum_{J} A_{J}^{*} A_{J}-1\right)\right. & \\
& \left.-\sum_{\kappa=1}^{p} \sum_{j, \ell=1}^{n_{\kappa}} \epsilon_{j \ell}^{(\kappa)}\left(\left\langle\varphi_{j}^{(\kappa)} \mid \varphi_{\ell}^{(\kappa)}\right\rangle-\delta_{j \ell}\right)\right\}=0,
\end{aligned}
$$

where it is assumed that $\Psi$ is of MCTDH form, Eq. (68). The Lagrange multipliers $E$ and $\epsilon_{j l}^{(\kappa)}$ are introduced to keep the $A$-vector normalized and the SPFs orthonormal.

Varying $A_{J}^{*}$ yields

$$
\sum_{L} \mathcal{K}_{J L} A_{L}=E A_{J}
$$

Hence the coefficient vector is obtained as an eigenvector of the Hamiltonian represented in the basis of the configurations $\Phi_{J}$.

From Eq. (76) it follows that a variation of the total wavefunction with respect to a SPF yields a single-hole function. Using this and varying the expression (85) with respect to $\left\langle\varphi_{k}^{(\kappa)}\right|$ gives $[187,188]$

$$
\sum_{l=1}^{n_{\kappa}} \mathcal{H}_{j \ell}^{(\kappa)} \varphi_{\ell}^{(\kappa)}=\sum_{\ell=1}^{n_{\kappa}} \epsilon_{j \ell}^{(\kappa)} \varphi_{\ell}^{(\kappa)} .
$$

Projecting Eq. (87) onto $\varphi_{k}^{(\kappa)}$, one arrives at

$$
\epsilon_{j k}^{(\kappa)}=\sum_{\ell=1}^{n_{\kappa}}\left\langle\varphi_{k}^{(\kappa)}\left|\mathcal{H}_{j \ell}^{(\kappa)}\right| \varphi_{\ell}^{(\kappa)}\right\rangle
$$

and from Eqs. $(87,88)$ it then follows

$$
\left(1-P^{(\kappa)}\right) \sum_{\ell=1}^{n_{\kappa}} \mathcal{H}_{j \ell}^{(\kappa)} \varphi_{\ell}^{(\kappa)}=0
$$


A variationally optimal solution is found when Eqs. $(86,89)$ are satisfied simultaneously.

Since Eq. (89) holds for each $j$, it must hold for any linear combination of these equations as well. To arrive at a form similar to the equation of motion (73) we insert the inverse of the density matrix

$$
\frac{\partial \varphi_{j}^{(\kappa)}}{\partial \tau}:=-\left(1-P^{(\kappa)}\right) \sum_{k, \ell=1}^{n_{\kappa}}\left(\rho^{(\kappa)}\right)_{j k}^{-1} \mathcal{H}_{k \ell}^{(\kappa)} \varphi_{\ell}^{(\kappa)}=0
$$

where $\tau=-i t$. This suggests a convenient way to solve the variational equation (89). The SPFs are relaxed, i.e. propagated in negative imaginary time, until their time derivative is sufficiently small.

The improved relaxation method proceeds as follows. First the user has to define an initial state which should have some overlap with the eigenstate one wants to compute. The Hamiltonian is then diagonalized in the basis of the configurations $\Phi_{J}$ of the initial state. After the diagonalization, the meanfields $\mathcal{H}^{(\kappa)}$ are built and the SPFs are relaxed over a suitable time interval. The Hamilton matrix $\mathcal{K}$ is then rebuilt in the new configurations (Hartree products) and diagonalized. The whole process is iterated until convergence is achieved. If the ground state is computed, the selection of the eigenvector of the Hamiltonian matrix is simple: one takes the eigenvector with lowest energy. When excited states are computed, that eigenvector is taken which corresponds to the wavefunction with largest overlap with the initial state.

As the dimension of the space spanned by the configurations is rather large - typical values range from 2,000 to 2,000,000 - the Davidson algorithm [136] is employed for the diagonalization of $\mathcal{K}$. The preconditioner for the Davidson step is, as usual, the diagonal of the matrix. When excited states are computed one may additionally improve the preconditioner by inverting a, say, $1000 \times 1000$, block around the energy of interest. This accelerates the convergence of the Davidson iterations.

An MCTDH propagation always works, whatever the numbers of SPFs. If there are too few configurations, the propagation will be less accurate but usually still describes the overall features rather well. This is in contrast to improved relaxation which fails to converge when the configuration space is too small. There is no problem in computing the ground state, this will always work, but converging to excited states becomes more difficult the higher the excitation energy, or - more precisely - the higher the density of states.

After each diagonalization of $\mathcal{K}$ one obtains an approximation to the sought exact eigenstate of the Hamiltonian. This approximate eigenstate contains, of course, contributions from many exact eigenstates. Problematic are here contributions from eigenstates with energies below the one sought. If these contributions are not small, the algorithm 'notices' that the energy can be lowered by optimizing the SPFs for the low-energy contributions to the approximate eigenstate. The energy hence decreases in the following improved relaxation 
iterations until - based on the overlap criterion - another eigenvector of $\mathcal{K}$ is taken. In this case, the energy jumps up and is lowered again in the following iteration steps. The energy keeps on oscillating [141] and a convergence cannot be achieved. Presently the only known solution to this problem is to increase the numbers of SPFs. A larger configuration space, however, quickly makes the calculation unfeasible, at least for large systems. Note that if convergence is achieved, the computed energies are variational, i. e. they are upper bounds to the exact ones $[189,190]$.

\subsection{Example}

Improved relaxation has been applied quite successfully to a number of problems, see e. g. Refs. [141,175,191]. For 4-atom systems (6D) it is in general possible to compute all eigenstates of interest. For a system as large as $\mathrm{H}_{5} \mathrm{O}_{2}^{+}$ (15D) it was only possible to converge low-lying states [175]. In this case filter diagonalization was used to compute the energies of high lying states.

The improved relaxation algorithm may be used in block form, i. e. one may start with a block of initial vectors which then converge collectively to a set of eigenstates. Formally the different wavefunctions are treated as electronic states of one 'super wavefunction'. The mean-fields are state-averaged mean-fields and the Davidson routine is replaced by a block-Davidson one.

As an example [192] we discuss the results of computing the lowest 40 eigenstates of HONO by block improved relaxation. The HONO molecule $[185,191,193]$ is described in valence coordinates. The six DOFs are combined to four particles where the first two (uncombined) particles represent the outof-plane torsion and the $\mathrm{O}-\mathrm{H}$ stretch. The underlying primitive grid consists of $28 \times 10^{6}$ points. The results of two calculations, using different numbers of SPFs, are shown in Tab. 2.

The first run used the SPF set 10/5/30/20 (i. e. 30,000 configurations altogether) and took $3 \mathrm{~h}$ CPU time and $560 \mathrm{MB}$ RAM on a $3.2 \mathrm{GHz}$ Pentium $4 \mathrm{PC}$, the second run with $12 / 5 / 42 / 28$ SPFs took $11 \mathrm{~h}$ CPU time and 1340 MB RAM. The second calculation was done mainly to show that already the first one is sufficiently converged. The energy is lowered by less than $0.3 \mathrm{~cm}^{-1}$ for all states except one, the 35 th excited state is lowered by $0.758 \mathrm{~cm}^{-1}$.

Block-relaxation requires more SPFs to converge than single-vector relaxation, because in block-relaxation the SPFs are optimized to represent all considered states simultaneously, while in single-vector relaxation they are optimized to represent a particular state. However, we found that the required increase in the number of SPFs is rather small. In fact, block-relaxation is in general more efficient than performing single-vector relaxations for each of the block states. But the memory consumption of block-relaxation is considerably larger (560 MB compared to $20 \mathrm{MB}$ for the present example). This may limit the applicability of block-improved-relaxation when turning to larger systems. 
Table 2: The lowest forty vibrational eigenvalues of HONO. The results of two calculations and their differences are displayed (see text). All energies are in $\mathrm{cm}^{-1}$. A ground state energy of $4367.634515 \mathrm{~cm}^{-1}$ is subtracted from all entries of column 2 and 3.

\begin{tabular}{rrrr}
$\#$ & $10 / 5 / 30 / 20$ & $12 / 5 / 42 / 28$ & diff \\
\hline 0 & 0.000 & 0.000 & .000 \\
1 & 93.974 & 93.972 & .002 \\
2 & 600.873 & 600.871 & .002 \\
3 & 710.625 & 710.621 & .004 \\
4 & 796.000 & 795.997 & .003 \\
5 & 944.116 & 944.108 & .008 \\
6 & 1055.385 & 1055.384 & .001 \\
7 & 1188.079 & 1188.070 & .009 \\
8 & 1267.609 & 1267.598 & .011 \\
9 & 1306.604 & 1306.595 & .009 \\
10 & 1312.761 & 1312.736 & .025 \\
11 & 1385.263 & 1385.247 & .016 \\
12 & 1405.545 & 1405.510 & .035 \\
13 & 1547.458 & 1547.431 & .027 \\
14 & 1574.851 & 1574.821 & .030 \\
15 & 1640.887 & 1640.884 & .003 \\
16 & 1690.034 & 1690.006 & .028 \\
17 & 1726.050 & 1726.009 & .041 \\
18 & 1761.638 & 1761.581 & .057 \\
19 & 1779.466 & 1779.377 & .089 \\
20 & 1829.017 & 1829.013 & .004 \\
21 & 1858.242 & 1858.210 & .032 \\
22 & 1897.807 & 1897.580 & .227 \\
23 & 1902.886 & 1902.838 & .048 \\
24 & 1961.701 & 1961.558 & .143 \\
25 & 2002.404 & 2002.323 & .081 \\
26 & 2025.384 & 2025.381 & .003 \\
27 & 2049.045 & 2048.967 & .078 \\
28 & 2120.019 & 2120.002 & .017 \\
29 & 2136.567 & 2136.276 & .291 \\
30 & 2154.080 & 2153.897 & .183 \\
31 & 2210.633 & 2210.622 & .011 \\
32 & 2240.933 & 2240.825 & .108 \\
33 & 2291.196 & 2291.096 & .100 \\
34 & 2306.477 & 2306.460 & .017 \\
35 & 2322.512 & 2321.754 & .758 \\
36 & 2339.333 & 2339.225 & .108 \\
37 & 2339.687 & 2339.416 & .271 \\
38 & 2370.709 & 2370.415 & .294 \\
39 & 2376.419 & 2376.401 & .017
\end{tabular}




\section{Applications}

We now present applications of these various approaches to $\mathrm{CH}_{4}, \mathrm{CH}_{3} \mathrm{OH}$, and $\mathrm{H}_{5} \mathrm{O}_{2}^{+}$.

\section{$8.1 \mathrm{CH}_{4}$}

First we present benchmark calculations of the vibrational energies of $\mathrm{CH}_{4}$ using the contracted-iterative method outlined in section 5. This method has been applied to several molecules. It was first tested on $\mathrm{HOOH}$ where its efficiency was established [157]. Yu has contracted the bend but not the stretch basis to compute energy levels for several molecules $[159,160,194]$. Lee and Light contracted both bend and stretch bases [161,162]. In this subsection we summarize calculations done on methane using the contracted basis-iterative method [54,158]. To compute vibrational energy levels of methane we use $4+1$ Radau vectors [195] and their associated polyspherical coordinates. There are three polar angles, $\theta_{i}(i=1,2,3)$ and two dihedral angles $\phi_{i}(i=2,3)$. The coordinates are defined explicitly in Ref. [158]. The calculation consists of three basic steps: 1) solve the stretch problem; 2) solve the bend problem; 3) combine solutions of the stretch and bend problems to determine energy levels of methane.

The stretch KEO is simple,

$$
T_{s t r}=\sum_{i=0}^{3}-\frac{1}{2 \mu_{i}} \frac{\partial^{2}}{\partial r_{i}^{2}} .
$$

Stretch eigenfunctions are computed with a primitive basis of products of 10 1D PODVR functions $[196,197]$. These in turn are built from a sinc DVR basis in the range $\left[1.2 a_{0}, 7.0 a_{0}\right][198]$. We discard all the PODVR points whose potential energy is above $30000 \mathrm{~cm}^{-1}$. This reduces the basis size from 10000 to 5049 . We retain all the stretch levels up to $20000 \mathrm{~cm}^{-1}$. We calculate stretch levels separately for the two irreps of $G_{6}$. This is done using the symmetry adapted Lanczos (SAL) algorithm. [199,200]

The bend KEO is easily extracted from Eq. (27). We use a parity adapted basis of products of spherical harmonic basis functions with $l_{\max }=m_{\max }=25$ and the SAL to compute eigenvalues and eigenvectors for different irreps of $G_{6}$ [201]. The spherical harmonic basis has the advantage that it enables one to easily compute matrix elements of the bend KEO using a very compact from (see Eq. (27)). It also makes it possible to calculate wavefunctions with nonzero amplitude at singularities of the bend KEO (not a problem for methane). Odd parity bend wavefunctions are computed and the SAL is used to obtain levels labelled by irreducible representations of the $G_{6}$ group. We retain bend eigenfunctions up to the polyad $n_{2}+n_{4}=6$, i.e., all those that differ from the bend ground state by less than $9098.0 \mathrm{~cm}^{-1}$. This basis is somewhat larger than the one used in Ref. [158]), but the lower levels in Ref. [158] are all well 
converged. $G_{2}$ symmetry is exploited when solutions of the bend and stretch subproblems are coupled to obtain the final energy levels. See Ref. [158] for more detail.

To use the eigenfunctions of the bend and stretch problems to compute energy levels and wavefunctions of methane we must compute and store them. Computing accurate eigenvalues with the Lanczos algorithm is extremely easy, but to obtain the corresponding eigenvectors one must be careful to limit the influence of numerical error. To do so one determines, separately for each eigenvector, the number of Lanczos vectors to be used to compute it. This is the iteration depth $[54,135]$. To store the eigenvectors we use a technique called compaction $[125,155,158]$. We need $\mathbf{C}$ (see section 5 ) to compute the final levels. Rather than storing $\mathbf{C}$ we store the important rows of $\tilde{\mathbf{C}}$, where

$$
\tilde{C}_{\beta b} \equiv \sum_{l} T_{l \beta} C_{l b}
$$

All elements of rows of $\tilde{C}_{\beta b}$ that correspond to quadrature points at which the potential is high will be small and can be discarded. For methane the ratio of the number of retained quadrature points to the number of direct product quadrature points is about 0.01. Storing the compacted version of $\tilde{\mathbf{C}}$ makes it much easier to use the contracted basis.

Table 3: Vibrational energy levels of $\mathrm{CH}_{4}$ in $\mathrm{cm}^{-1}$.

- Printer: Please put Table here! (to be found on last page where it is called TABLE I) -

We use the T8 potential surface described in Ref. [202] The size of the basis of products of stretch and bend functions is 113360 . These functions are divided into two symmetry groups. There are $57548 \mathrm{~A}$ symmetry functions and $55812 B$ symmetry functions. The final basis set is about 6 orders of magnitude smaller than the original primitive basis! Energy levels are reported in Table 3. The column labelled Basis 1 is obtained with basis 1 of Ref. [158]. Levels above about $5100 \mathrm{~cm}^{-1}$ are better converged than those given in Ref. [158] where not all the bend basis functions in the $n_{2}+n_{4}=6$ polyad were included. The states for which the energy in Ref. [158] differs most from the energy reported here are those with the largest $n_{2}+n_{4}$ values. Many high stretch overtone and combination states are also well converged but are not included in the table. There is good agreement between experimental and computed levels, indicating that the potential is realistic.

Using this contracted basis-iterative eigensolver approach it has also been possible to compute vibrational energy levels of $\mathrm{CH}_{5}^{+}$. The details of this calculation will be published soon [203]. As was done for methane we use Radau vectors and their associated angles and a large spherical harmonics basis. A primitive bend basis with more than 200 million functions is required to con- 
verge the bend levels. The energy level pattern differs from those obtained previously.

These results for $\mathrm{CH}_{4}$ were recently used as benchmarks to test the accuracy of vibrational energies obtained using MULTIMODE with $n M R, n=2,3,4,5$ representations of the potential (and the vibrational angular momentum terms) [204]. The MULTIMODE calculations did not make full use of the $T_{d}$ symmetry and instead were done with $C_{2 v}$ symmetry. Rather than repeat the lengthy table of comparisons in Ref. [204] we graphically show the convergence and accuracy of MULTIMODE energies as n varies by plotting the root mean square (rms) deviation from the benchmark energies in Fig.1 As seen the rms differ-

Figure 1: Root mean square difference between nMR MULTIMODE energies and benchmark ones [158] versus n for roughly 50 energies $4,500 \mathrm{~cm}^{-1}$ above the zero-point energy.

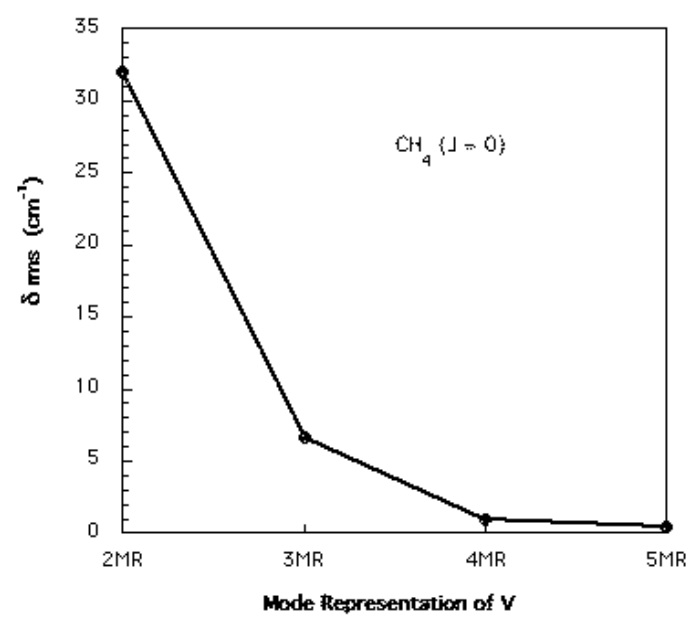

ence decreases by roughly an order of magnitude from $32 \mathrm{~cm}^{-1}$ for the $2 \mathrm{MR}$ calculations to less than $0.5 \mathrm{~cm}^{-1}$ for the $5 \mathrm{MR}$ calculations which are thus in quite good agreement with the benchmark energies. For several states the error in the 2MR calculations is between 70 and $80 \mathrm{~cm}^{-1}$. The sizes of the four VCI matrices, obtained with a direct-product basis but with excitation restrictions, for the $4 \mathrm{MR}$ and $5 \mathrm{MR}$ calculations were 54522, 47312, 47312 and 42459. These are moderately large and and so the block Davidson method was used to get the eigenvalues. We also examined the degeneracy of $E$ and $F$ fundamentals and showed that correct degeneracy to within less than $1 \mathrm{~cm}^{-1}$ was achieved with a $4 \mathrm{MR}$ and to within less than $0.1 \mathrm{~cm}^{-1}$ with $5 \mathrm{MR}$.

As noted above MULTIMODE can also perform VCI calculations for $J>$ 0 and energies $\left(\mathrm{cm}^{-1}\right)$ for $J=1$ agree well with the benchmark results, as shown in Table 4.

Next we briefly consider the most recent MULTIMODE-RPH calculations 
Table 4: Comparison of 5MR MULTIMODE rovibrational energies of $\mathrm{CH}_{4}$ $\left(\mathrm{cm}^{-1}\right)$ for $J=1$ with benchmark results of Ref. [158]

\begin{tabular}{ccc}
\hline \hline State & Benchmark & MULTIMODE \\
\hline zero point & 10.43 & 10.43 \\
$(00)(01) \mathrm{A} 2$ & 1312.41 & 1312.50 \\
$(00)(01) \mathrm{F} 2$ & 1317.25 & 1317.34 \\
$(00)(01) \mathrm{F} 1$ & 1326.73 & 1326.82 \\
$(00)(01) \mathrm{E}$ & 1327.03 & 1327.12 \\
$(00)(10) \mathrm{F} 2$ & 1543.79 & 1543.88 \\
$(00)(10) \mathrm{F} 1$ & 1543.91 & 1544.01 \\
$(00)(02) \mathrm{F} 1$ & 2600.02 & 2600.58 \\
$(00)(02) \mathrm{F} 1$ & 2615.61 & 2616.36 \\
$(00)(02) \mathrm{E}$ & 2622.05 & 2622.82 \\
$(00)(02) \mathrm{F} 2$ & 2626.64 & 2627.37 \\
$(00)(02) \mathrm{A} 2$ & 2634.83 & 2635.61 \\
$(00)(02) \mathrm{F} 2$ & 2641.58 & 2642.25 \\
$(00)(02) \mathrm{F} 1$ & 2644.03 & 2644.68 \\
$(00)(11) \mathrm{E}$ & 2835.80 & 2836.67 \\
$(00)(11) \mathrm{F} 1$ & 2837.85 & 2838.73 \\
$(00)(11) \mathrm{F} 2$ & 2843.53 & 2844.39 \\
$(00)(11) \mathrm{A} 2$ & 2848.87 & 2849.75 \\
$(00)(11) \mathrm{F} 2$ & 2856.65 & 2857.36 \\
$(00)(11) \mathrm{E}$ & 2858.02 & 2858.73 \\
$(00)(11) \mathrm{F} 1$ & 2860.39 & 2861.11 \\
$(00)(11) \mathrm{A} 1$ & 2862.34 & 2863.06 \\
$(10)(00) \mathrm{F} 1$ & 2924.16 & 2924.32 \\
$(01)(00) \mathrm{A} 2$ & 3022.81 & 3023.02 \\
$(01)(00) \mathrm{F} 2$ & 3023.36 & 3023.58 \\
$(01)(00) \mathrm{F} 1$ & 3024.50 & 3024.72 \\
$(01)(00) \mathrm{E}$ & 3024.56 & 3024.78 \\
$(00)(20) \mathrm{F} 1$ & 3074.26 & 3074.68 \\
$(00)(20) \mathrm{F} 2$ & 3075.80 & 3076.22 \\
$(00)(20) \mathrm{F} 1$ & 3075.82 & 3076.50 \\
\hline \hline
\end{tabular}

for $\mathrm{CH}_{3} \mathrm{OH}$ [91]. After the validation of the MM-RPH approach for $\mathrm{H}_{2} \mathrm{O}_{2}$ [87] methanol was one of the first new applications of the method, [88], using a fairly low-level of electronic structure obtained along the reaction path, i.e., the torsion coordinate. In the latest work a semi-global potential energy surface fit to roughly $20000 \mathrm{CCSD}(\mathrm{T})$ /aug-cc-pVTZ energies was used [91]. Convergence studies indicated that the energies are converged to within a $\mathrm{cm}^{-1}$ or less with a $5 \mathrm{MR}$ for the potential and a $3 \mathrm{MR}$ for vibrational angular momentum terms. This is quite impressive given that this is a floppy 12 degree of freedom molecule.

A sample of the energies and comparison with experiment is given in Ta- 
Table 5: Comparison of experimental and MULTIMODE-RPH vibrational energies $\left(\mathrm{cm}^{-1}\right)$ and splittings (right section of the table) of $\mathrm{CH}_{3} \mathrm{OH}$. Lefthand entries are the non-degenerate $A_{1}$ and $A_{2}$ levels in the $G_{6}$ point group arising from the MM-RPH $\mathrm{A}^{\prime}$ and $\mathrm{A}^{\prime \prime}$ levels from calculations in $\mathrm{C}_{s}$ symmetry, respectively. Right-hand entries are the relative energies of the corresponding degenerate $\mathrm{E}$ levels in the $\mathrm{G}_{6}$ point group.

\begin{tabular}{cccc|cc}
\hline \hline Level & Symm. & Expt. & $M M-$ RPH & Expt. & $M M-R P H$ \\
\hline$\nu_{8}$ & $A_{1}$ & 1033.8 & 1026.3 & 8.27 & 10.50 \\
$\nu_{7}$ & $A_{1}$ & 1074.5 & 1079.9 & 4.61 & 7.62 \\
$\nu_{6}$ & $A_{1}$ & 1335.8 & 1321.9 & $/$ & 20.01 \\
$\nu_{5}$ & $A_{1}$ & 1454.5 & 1446.8 & $/$ & 8.72 \\
$\nu_{4}$ & $A_{1}$ & 1486.4 & 1483.8 & $/$ & -2.72 \\
$\nu_{3}$ & $A_{1}$ & 2844.2 & 2839.5 & 9.07 & 9.09 \\
$\nu_{2}$ & $A_{1}$ & 2999.0 & 2986.3 & -3.26 & -0.68 \\
$\nu_{1}$ & $A_{1}$ & 3683.9 & 3675.0 & 6.30 & 6.90 \\
$2 \nu_{8}$ & $A_{1}$ & 2054.7 & 2038.7 & 8.90 & 12.85 \\
$2 \nu_{7}$ & $A_{1}$ & 2140.6 & 2152.5 & +2.29 & +5.67 \\
$2 \nu_{6}$ & $A_{1}$ & 2632.0 & 2678.4 & $/$ & -26.47 \\
$\nu_{7}+\nu_{8}$ & $A_{1}$ & 2097.3 & 2097.6 & +4.65 & +6.36 \\
$\nu_{11}$ & $A_{2}$ & 1164.0 & 1156.5 & -7.43 & -5.42 \\
$\nu_{10}$ & $A_{2}$ & 1481.8 & 1475.1 & $/$ & -4.24 \\
$\nu_{9}$ & $A_{2}$ & 2966.6 & 2961.5 & -5.48 & -3.43 \\
$\nu_{8}+\nu_{12}$ & $A_{2}$ & 1231.6 & 1234.9 & -76.93 & -56.74 \\
$\nu_{8}+\nu_{11}$ & $A_{2}$ & 2190.6 & 2176.7 & -7.70 & -3.19 \\
\hline
\end{tabular}

ble 5. As seen there is good agreement with experiment and the pattern of tunneling splittings is well reproduced by the calculations.

\subsection{Vibrational Spectroscopy of the Zundel cation}

The Zundel cation $\left(\mathrm{H}_{5} \mathrm{O}_{2}^{+}\right)$is the smallest protonated water cluster in which an excess proton is shared between different water molecules. This species has been the target of much research effort during the last years owing to its key role as a limiting structure of the hydrated proton in bulk water. The development and improvement of new experimental techniques to measure accurate IR spectra of ionic species in the gas phase has led to an accurate characterization of the IR spectrum of $\mathrm{H}_{5} \mathrm{O}_{2}^{+}$[205-209]. Several theoretical studies have appeared in parallel to provide the necessary basis for the rationalization and understanding of the measured spectra. In particular 4D simulations that included the position of the central proton $(x, y, z)$ and the oxygen-oxygen distance $(R)$ can be found in the literature [210]. Those simulations yielded a too high frequency for the fundamental excitation of the central proton along the $\mathrm{O}-\mathrm{O}$ axis (asymmetric stretch $\nu_{a p}$ ), while the out-of-axis $(x, y)$ motions of 
the proton presented too low frequencies, to the extent that the frequencies of these states presented an inverted ordering pattern. VCI calculations showed later that a full dimensional treatment would be necessary to regain the correct ordering of the fundamental modes related to the central proton due to the strong coupling of these motions to displacements of the surrounding water molecules $[208,211]$. The IR spectrum of the Zundel cation recorded with the messenger atom technique features a doublet structure in the spectral region around $1000 \mathrm{~cm}^{-1}$ with a splitting of about $120 \mathrm{~cm}^{-1}$ [208]. The origin of the doublet remained an unsolved issue that could not be reproduced nor explained by means of VCI [208] nor by classical dynamics simulations [212,213], although there was accumulated evidence that the $\nu_{a p}$ fundamental would be mostly responsible for the highest intensity line of the doublet. Moreover, the lowest frequency region of the spectrum below $800 \mathrm{~cm}^{-1}$, where the fundamental states of the most anharmonic motions are found, had not yet been accessed experimentally. Other lines appearing between 1000 and $2000 \mathrm{~cm}^{-1}$ had not been yet conclusively assigned and their origin was unclear. This inherently complex nature of $\mathrm{H}_{5} \mathrm{O}_{2}^{+}$is caused by the floppy, anharmonic nature of the interatomic potential governing the motion of the cation, in which internal rotation and long range angular motions take place at relatively low vibrational energies. In this context the study of the IR spectroscopy and dynamics of the Zundel cation was undertaken by the MCTDH method [175, 214,215].

\subsubsection{Setup of the Hamiltonian Operator}

The simulation of floppy systems like the Zundel cation requires the use of a proper set of coordinates that is able to describe largely anharmonic, angular motions, e.g. torsions or internal rotations. To this end a set of polyspherical vectors is used to describe the configuration of the system. For a detailed derivation of the KEO in the set of polyspherical coordinates used for $\mathrm{H}_{5} \mathrm{O}_{2}^{+}$ see Ref. [215]. The set of polyspherical vectors that are used to describe the $\mathrm{H}_{5} \mathrm{O}_{2}^{+}$cation are shown in Fig. 2. This is a semirigid system (at least in the range of vibrational energies involved in linear the IR spectrum), i.e., no singularities in the kinetic energy exist for the configurations accessible to the system. This allows the use of standard one-dimensional DVRs. (see Appendix in Ref. [215]). The 15 coordinates are defined as follows: the distance between the centers of mass of both water molecules $(R)$, the position of the central proton with respect to the center of mass of the water dimer $(x, y, z)$, the Euler angles defining the relative orientation between the two water molecules (waggings: $\gamma_{A}, \gamma_{B}$; rockings: $\beta_{A}, \beta_{B}$; internal relative rotation: $\alpha$ ) and the Jacobi coordinates which account for the particular configuration of each water molecule $\left(R_{1(A, B)}, R_{2(A, B)}, \theta_{(A, B)}\right)$ where $R_{1 x}$ is the distance between the oxygen atom and the center of mass of the corresponding $\mathrm{H}_{2}$ fragment, $R_{2 x}$ is the $\mathrm{H}-\mathrm{H}$ distance and $\theta_{x}$ is the angle between these two vectors.

The generation of a potential energy operator (PEO), i.e., a working ap- 
Figure 2: Jacobi description of the $\mathrm{H}_{5} \mathrm{O}_{2}^{+}$system. The vector $\mathbf{R}$ connects the two centers of mass of the water monomers. The position of the central proton is measured from the center of mass of the water dimer.

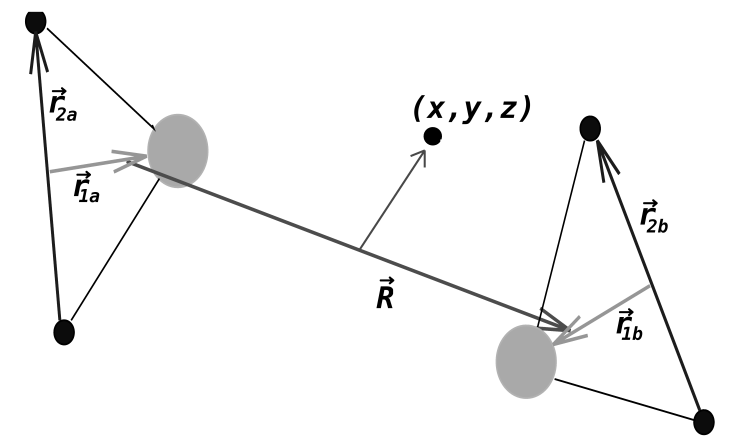

proximation to the full PES, is a rather challenging task of its own, in particular for problems as large as the present one (15D). A way to represent such high-dimensional surfaces is the so-called Cartesian reaction surface (CRS) approach, in which a small number of coordinates (typically 2) are selected as defining the reaction surface while the potential for the rest of the coordinates is given as a second order Taylor expansion whose coefficients are dependent on the position of the coordinates in the first group. The CRS approach has the required product form, Eq. (3), meaning that it is an efficient PESrepresention for MCTDH, and in fact it has been used in a number of MCTDH applications [216]. Since the approach is based on orthogonal, rectilinear coordinates, the KEO is simply diagonal. Such an approximation can be used if a large number of coordinates behave almost harmonic and they are only weakly coupled among each other and to the reaction coordinates. Such an approximation breaks down however for loosely bonded systems that feature relatively soft, anharmonic potentials. Protonated water clusters belong to this kind of systems and the relatively straightforward CRS is therefore not applicable.

Another possibility to efficiently represent the $15 \mathrm{D} \operatorname{PES} V(\mathbf{Q})$ is to use a variant of the n-mode representation of the potential given by Eq. (2). In a similar way to the definition of the MCTDH ansatz, Eq. (68), it is advantageous to work here with combined ("logical") coordinates instead of uncombined ("physical") coordinates when defining the PES expansion. The logical $Q_{\kappa}$ coordinates, defined in Eq (69), refer then to composite coordinates of one or more physical coordinates. In this case we call the $V^{(n)}$ terms combined clusters $(\mathrm{CC})$. The numerical advantage in the representation of the PES obtained when using combined coordinates instead of single coordinates is analyzed in Ref. [215]. The definition of meaningful mode combinations should be possible in most cases. Highly correlated coordinates should be grouped together so 
that their correlation is taken into account already by the first order CCs. Such a definition would be based on chemical common-sense, e.g., coordinates belonging to the same chemical group or to the same molecule in a cluster are good candidates to be combined. The application of this scheme does not only lead to numerical advantage in the representation of the PES with respect to the uncombined scheme. Its application in MCTDH will be (much) more efficient if the mode combination used to define the PES is the same that is used in defining the wavefunction. In such a case the first order CCs $V^{(1)}\left(Q_{i}\right)$ are full product grids defined in the space of the $Q_{i}$ combined coordinate. The higher order terms are given by potfit representations of the total product grids $V^{(n>1)}\left(Q_{i}, \ldots\right)$. A potfit representation can be defined in terms of combined modes and in such a case the SPPs are product grids in the space of each $Q_{i}$. This strategy can efficiently be applied as long as the full product grid of the $n$-th order cluster fits into memory. In practice this means around 7 single coordinates, roughly corresponding to CCs of third order. As a final remark, the convergence of the $n$-mode representation is highly dependent on the set of coordinates used and the grouping of coordinates into particles. With our set of polyspherical coordinates, which correctly account for the fundamental motions of the cation in terms of rotations, torsions, etc., and a clever grouping of the coordinates we obtain a relatively good potential already with a second order expansion. Further details are given in the following section.

\subsubsection{Representation of the Potential Energy Surface for $\mathrm{H}_{5} \mathrm{O}_{2}^{+}$}

The PEO for the $\mathrm{H}_{5} \mathrm{O}_{2}^{+}$system is constructed using the full-dimensional PES developed by Huang et. al. [217]. Such a PES is based on several ten thousands of Coupled Cluster energy calculations which are combined with a clever fitting algorithm based on polynomials that respect the total permutational symmetry of the system. In order to construct an efficient representation of that PES for the $\mathrm{H}_{5} \mathrm{O}_{2}^{+}$cation employing the approach described above one must start by defining the combined modes, also called particles, that shall be used. In the present case the following five multidimensional modes are selected: $Q_{1}=[z, \alpha, x, y], Q_{2}=\left[R, u_{\beta_{A}}, u_{\beta_{B}}\right], Q_{3}=\left[\gamma_{A}, \gamma_{B}\right], Q_{4}=\left[R_{1 A}, R_{2 A}, u_{\theta_{1 A}}\right]$ and $Q_{5}=\left[R_{1 B}, R_{2 B}, u_{\theta_{1 B}}\right]$ where $u_{x}=\cos x$. It is convenient that coordinates $x, y$ and $\alpha$ are grouped together due to symmetry conserving reasons [215]. Modes $Q_{2}$ and $Q_{3}$ contain the rocking and wagging coordinates, respectively. Modes $Q_{4}$ and $Q_{5}$ contain the Jacobi coordinates representing the internal configuration of each water molecule. The specific form of the used PEO is given by

$$
\tilde{V}(\mathbf{Q})=V^{(0)}+\sum_{i=1}^{5} V_{i}^{(1)}\left(Q_{i}\right)+\sum_{i=1}^{4} \sum_{j=i+1}^{5} V_{i j}^{(2)}\left(Q_{i}, Q_{j}\right)+V_{z 23}^{(3)}\left(z, Q_{2}, Q_{3}\right),
$$

where the modes $Q_{1} \cdots Q_{5}$ have been defined above. The $V^{(0)}$ term is the energy at the reference geometry. The $V_{i}^{(1)}$ terms are the intra-group potentials 
Table 6: Expectation value of the different terms of the potential expansion (central column) and square root of the expectation value of the potential squared (right column). All energies in $\mathrm{cm}^{-1}$. The combined modes read: $Q_{1}=[z, \alpha, x, y], Q_{2}=\left[R, u_{\beta_{A}}, u_{\beta_{B}}\right], Q_{3}=\left[\gamma_{A}, \gamma_{B}\right], Q_{4}=\left[R_{1 A}, R_{2 A}, u_{\theta_{1 A}}\right]$ and $Q_{5}=\left[R_{1 B}, R_{2 B}, u_{\theta_{1 B}}\right] \cdot u_{x}=\cos x, \gamma$ refers to wagging and $\beta$ refers to rocking motion. $Q_{4}$ and $Q_{5}$ contain the Jacobi coordinates of the water molecules.

\begin{tabular}{lcc}
\hline \hline & $\left\langle\Psi_{0}|V| \Psi_{0}\right\rangle$ & $\left\langle\Psi_{0}\left|V^{2}\right| \Psi_{0}\right\rangle^{1 / 2}$ \\
\hline$V^{(1)}\left(Q_{1}\right)$ & 1293.6 & 1807.7 \\
$V^{(1)}\left(Q_{2}\right)$ & 750.6 & 966.9 \\
$V^{(1)}\left(Q_{3}\right)$ & 171.5 & 266.9 \\
$V^{(1)}\left(Q_{4}\right)$ & 2293.2 & 3062.8 \\
$V^{(1)}\left(Q_{5}\right)$ & 2293.1 & 3062.8 \\
$V^{(2)}\left(Q_{1}, Q_{2}\right)$ & -526.9 & 1037.2 \\
$V^{(2)}\left(Q_{1}, Q_{3}\right)$ & -78.8 & 290.2 \\
$V^{(2)}\left(Q_{1}, Q_{4}\right)$ & -27.5 & 231.8 \\
$V^{(2)}\left(Q_{1}, Q_{4}\right)$ & -27.4 & 231.7 \\
$V^{(2)}\left(Q_{2}, Q_{3}\right)$ & -10.5 & 37.6 \\
$V^{(2)}\left(Q_{2}, Q_{4}\right)$ & -24.7 & 117.5 \\
$V^{(2)}\left(Q_{2}, Q_{5}\right)$ & -24.7 & 117.9 \\
$V^{(2)}\left(Q_{3}, Q_{4}\right)$ & -18.8 & 180.9 \\
$V^{(2)}\left(Q_{3}, Q_{5}\right)$ & -18.8 & 180.9 \\
$V^{(2)}\left(Q_{4}, Q_{5}\right)$ & 1.2 & 9.9 \\
$V^{(3)}\left(z, Q_{2}, Q_{3}\right)$ & 1.0 & 50.4 \\
\hline \hline
\end{tabular}

obtained by keeping the coordinates in other groups at the reference geometry, while the $V_{i j}^{(2)}$ terms account for the group-group correlations. The potential with up to second-order terms gives already a very reasonable description of the system. The $V_{z 23}^{(3)}$ term accounts for three-mode correlations between the displacement of the central proton, the distance between both water molecules and the angular wagging and rocking motions. To ensure the full symmetry of the PES, an average over several reference geometries was used. For details see Ref. [215].

The quality of the PES expansion can be assessed by monitoring the expectation values of the different terms of the $n$-mode representation with respect to the ground vibrational state $\left|\Psi_{0}\right\rangle$. These values are given in $\mathrm{cm}^{-1}$ in Table 6 . The sum of the first order $\left\langle\Psi_{0}\left|V^{(1)}\left(Q_{i}\right)\right| \Psi_{0}\right\rangle$ terms is close to $6800 \mathrm{~cm}^{-1}$, half the ZPE, indicating that they carry the major weight in the description of the PES. The second order clusters introduce the missing correlation between modes. They have expectation values one order of magnitude smaller than the first order terms with one exception, the matrix element arising from the 
Table 7: Comparison of the zero point energy (ZPE) of the $\mathrm{H}_{5} \mathrm{O}_{2}^{+}$cation calculated by various approaches on the PES by Huang et al. [217]: diffusion Monte-Carlo (DMC), normal mode analysis (harmonic), vibrational CI single reference (VCI-SR) and reaction path (VCI-RP) as published in [218] and MCTDH results. $\Delta$ denotes the difference to the DMC result. The converged MCTDH result is obtained with 10500000 configurations. Compare with Table 8.

\begin{tabular}{ccc}
\hline \hline Method & $\mathrm{ZPE}\left(\mathrm{cm}^{-1}\right)$ & $\Delta\left(\mathrm{cm}^{-1}\right)$ \\
\hline DMC & 12393 & 0 \\
harmonic & 12635 & 242 \\
VCI-SR & 12590 & 197 \\
VCI-RP & 12497 & 104 \\
MCTDH & 12376.3 & -16.7 \\
\hline
\end{tabular}

$V^{(2)}\left(Q_{1}, Q_{2}\right)$ potential. This can be easily understood by noting that modes $Q_{1}$ and $Q_{2}$ contain coordinates $z$ and $R$, respectively. These two coordinates are strongly correlated and indeed they would be good candidates to be put in the same mode in an alternative mode-combination scheme. The only third order term that was introduced presents a rather marginal contribution to the potential energy of the system. These values prove that the PES representation used is of a good quality and rather well converged with respect to the reference PES, at least for the energy domain of interest. The square root of the expectation value of the potential squared is depicted in the third column. It is a measure of the dispersion around the expectation value and should also ideally vanish. The values indicate that the PES representation is good, albeit not yet fully converged.

\subsubsection{Ground Vibrational State and Eigenstates in the Low Fre- quency Domain}

The ground vibrational state and some eigenstates of the vibrational Hamiltonian are computed using the improved relaxation algorithm presented above, Sec. 7.4. The comparison between the largest, converged MCTDH calculation and other reported results on the same PES is given in Table 7. As a reference we take the given diffusion Monte Carlo (DMC) result [218], which has an associated statistical uncertainty of $5 \mathrm{~cm}^{-1}$. The most comprehensive calculations on the vibrational ground state based on a wavefunction approach prior to the MCTDH calculations were done by some of us [218] using the Multimode program [76]. The VCI results, both using the single reference (SR) and reaction path (RP) variants are found in Table 7. These calculations use a normal-mode based Hamiltonian. They incorporate correlation 
Table 8: Comparison of the zero point energy (ZPE) of the $\mathrm{H}_{5} \mathrm{O}_{2}^{+}$cation between different MCTDH calculations with ascending number of configurations. The $\Delta$ values are given with respect to the diffusion Monte Carlo result, 12393 $\mathrm{cm}^{-1}[218]$.

\begin{tabular}{crcc}
\hline \hline SPFs per Mode & N configs. & ZPE $\left(\mathrm{cm}^{-1}\right)$ & $\Delta\left(\mathrm{cm}^{-1}\right)$ \\
\hline$(20,20,12,6,6)$ & 172800 & 12383.7 & -9.3 \\
$(35,25,15,8,8)$ & 840000 & 12378.5 & -14.5 \\
$(40,40,20,8,8)$ & 2048000 & 12377.8 & -15.2 \\
$(60,40,20,8,8)$ & 3072000 & 12376.7 & -16.3 \\
$(70,50,30,10,10)$ & 10500000 & 12376.3 & -16.7 \\
\hline \hline
\end{tabular}

between the different degrees of freedom due to the n-mode representation of the potential [76] and the use of a CI wavefunction. The best reported VCI result for the ZPE lies still $104 \mathrm{~cm}^{-1}$ above the DMC value. It is worth to mention that before switching to a Hamiltonian based on polyspherical coordinates a Hamiltonian expressed in rectilinear coordinates was tried together with MCTDH and the obtained results similar to those obtained by VCI. The MCTDH converged result for the ZPE is given in Table 7 . The obtained value for the ZPE is $12376.3 \mathrm{~cm}^{-1}, 16.7 \mathrm{~cm}^{-1}$ below the DMC value. Table 8 contains ZPE values obtained using an increasing number of configurations. According to these results the MCTDH reported values are assumed to be fully converged with respect to the number of configurations. The deviation from the DMC result must be attributed to the cluster expansion (n-mode representation) of the potential. Table 8 illustrates an interesting property of MCTDH, namely its early convergence. The difference in energy between the computation on the top and the most expensive one below is only about 7 $\mathrm{cm}^{-1}$, yet the first calculation can be performed in a laptop while the most expensive one needed several days in a workstation. The variational optimality of the MCTDH equations, in particular the optimality of the SPFs, leads to qualitatively correct results as soon as there are enough SPFs to describe the physics of the problem at hand, while more accurate results can be obtained at the cost of increased computational resources.

The convergence depicted in Table 8 , however, is unusually slow. We attribute this to a "hole" in the PEO, i. e. a region where the potential is too low. Such holes are unfortunately rather common in n-mode representations. They appear at regions where almost all coordinates are far from their equilibrium position. Then many configurations are needed to enable the wavefunction to leak into the hole making the energy too low.

In the following we analyze the excited vibrational states related to the wagging motion due to the relevance of some of these modes in shaping the 
IR spectrum, as discussed in the next section. Further details on the internal rotation motion can be found in Ref. [215]. These states were computed by improved relaxation. Figure 3 depicts the probability density projected onto the wagging coordinates of the four lowest excited states related to the wagging motion. The shapes of these density plots make clear that the wagging motion takes place in a very anharmonic region of the PES. State $w_{1 a, b}$, which is a doubly degenerate state of an $E$ irreducible representation, is responsible for the absorption at about $100 \mathrm{~cm}^{-1}$ in the IR spectrum. In case the system is considered in its full $\mathcal{G}_{16}$ symmetry also the rotational motion around $\alpha$ is involved in the state corresponding to such absorption [215]. However, in this text we consider the symmetry labels as corresponding to the more familiar $\mathrm{D}_{2 d}$ point group. The energies of the next three wagging-mode states $\left(w_{2}, w_{3}, w_{4}\right)$ are, respectively, 232, 374 and $422 \mathrm{~cm}^{-1}$ and they are shown in Figs. 3b, 3c and $3 \mathrm{~d}$, respectively. These three states correspond to two quanta of excitation in the wagging motions and they can be represented by kets $|11\rangle,(|20\rangle-|02\rangle) / \sqrt{2}$ and $(|20\rangle+|02\rangle) / \sqrt{2}$, respectively, where the $|a b\rangle$ notation signifies the quanta of excitation in the wagging motions of monomer $A$ and $B$. These states have symmetries $B_{1}, B_{2}$ and $A_{1}$, respectively. In the harmonic limit these three states would be degenerate. The $w_{3}$ state has four probability-density maxima along the 2D space spanned by $\gamma_{A}$ and $\gamma_{B}$. They correspond to geometries in which one of the water molecules adopts a planar geometry $\left(\mathrm{H}_{2} \mathrm{O}\right.$ character $)$ and the other adopts a pyramidal geometry $\left(\mathrm{H}_{3} \mathrm{O}^{+}\right.$character $)$. This state transforms according to the $B_{2}$ symmetry representation, which is also the symmetry of the proton-transfer fundamental. State $w_{3}$ will play a major role due to its strong coupling to the proton-transfer mode.

\subsubsection{Infrared Absorption Spectrum}

The IR spectrum calculated with MCTDH is shown in Fig. 4. The MCTDH spectrum in the full range $0-4000 \mathrm{~cm}^{-1}$ is depicted in Fig. 4 (left panel) and compared to the recent experimental results of Ref. [209] in Fig. 4 (right panel). The dipole-moment operated ground state $\hat{\mu}\left|\Psi_{0}\right\rangle$ was propagated for $500 \mathrm{fs}$, yielding an autocorrelation of $1000 \mathrm{fs}$. The spectrum was calculated according to Eq. (81) and the FWHM resolution of the spectrum is, according to Eq. (84), about $30 \mathrm{~cm}^{-1}$.

Even if the computation of the IR spectrum in the time-dependent representation is feasible, a means of assigning the different lines to specific motions of the system is still required. Wavefunctions of excited states converged by improved relaxation, contain all the possible information on that specific state. The intensity of a given excited state $\left|\Psi_{n}\right\rangle$ is readily obtained by computing the dipole moment $\left|\left\langle\Psi_{n}|\hat{\mu}| \Psi_{0}\right\rangle\right|^{2}$. But even if an excited state of interest $\left|\Psi_{n}\right\rangle$ has been obtained it is difficult to directly inspect these mathematical objects due to their high dimensionality. Moreover, for the higher excited states we do not have $\left|\Psi_{n}\right\rangle$ at our disposal but only an autocorrelation function provid- 
Figure 3: Reduced probability density on the wagging coordinates $\gamma_{A}$ and $\gamma_{B}$ of (a) the first-excited $\left(w_{1 a, b}\right)$ wagging-mode state and (b), (c), (d), excited states $w_{2}, w_{3}$ and $w_{4}$ respectively, characterized by two quanta of excitation. In ket notation these four states can be represented as $w_{1 a, b}=|10\rangle \pm|01\rangle$, $w_{2}=|11\rangle, w_{3}=|20\rangle-|02\rangle$ and $w_{4}=|20\rangle+|02\rangle$, respectively, where the numbers represent the quanta of excitation in the wagging motion of each water molecule in a harmonic limit.

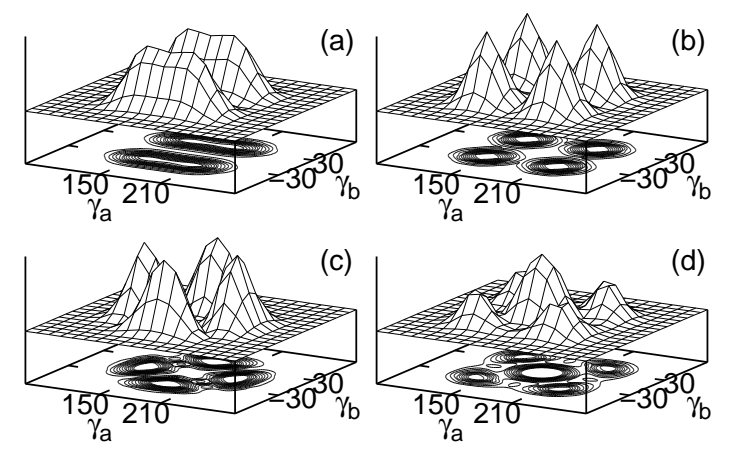

ing spectral lines. In both cases one can characterize the eigenstates by their overlaps with carefully chosen test states, i.e., by the numbers $\left|\left\langle\Phi_{\text {test }} \mid \Psi_{n}\right\rangle\right|^{2}$. The following procedures are used:

1. Test states $\left|\Phi_{\text {test }}\right\rangle$ are generated

a) by applying some operator $\hat{O}$ to a previously converged eigenfunction,

$$
\left|\Phi_{\text {test }}\right\rangle=N \hat{O}\left|\Psi_{n}\right\rangle
$$

where $N$ is a normalization constant, e.g., $N \hat{z}\left|\Psi_{0}\right\rangle$ generates a test state which in essence differs from the ground state $\left|\Psi_{0}\right\rangle$ by a one quantum excitation in the proton-transfer coordinate $z$.

b) by forming Hartree products, where the SPFs are obtained through diagonalization of mode-Hamiltonians $\hat{h}_{\kappa}$. The $\hat{h}_{\kappa}$ are low-dimensional Hamiltonians obtained from the full Hamiltonian by freezing the remaining coordinates. Each $\hat{h}_{\kappa}$ operates on the coordinate-space of a MCTDH-particle. Rather than using single Hartree products one may use linear combinations of a few products in order to satisfy a symmetry constraint.

2. The overlaps $\left|\left\langle\Phi_{\text {test }} \mid \Psi_{n}\right\rangle\right|^{2}$ are then computed by

a) by direct evaluation of the scalar product if $\left|\Psi_{n}\right\rangle$ is available.

b) by Fourier transform of the autocorrelation function $a(t)=\left\langle\Phi_{\text {test }}|\exp (-i H t)| \Phi_{\text {test }}\right\rangle$. The overlap is obtained via the 
Figure 4: Left panel: Simulated MCTDH spectrum in the range $0-4000 \mathrm{~cm}^{-1}$. Excitation in the $z$ direction (top), perpendicular to the O-H-O axis (middle) and total spectrum, i.e. $(1 / 3) z+(2 / 3) *$ perpendicular (bottom). Note the different scale of intensities in the perpendicular-component plot. Autocorrelation time $T=1000$ fs. Right panel: Comparison between the MCTDH spectrum (top) and the $\mathrm{H}_{5} \mathrm{O}_{2}^{+}$.Ne spectrum of Ref. [208] (bottom). The intensity of the experimental spectrum is adjusted in each spectral region (800-2000 and $3500-3800 \mathrm{~cm}^{-1}$ ) using the most intense peak of the MCTDH spectrum as a reference. Absorption for the MCTDH spectrum is given in absolute scale in mega-barns. $\left(1 \mathrm{Mb}=10^{-18} \mathrm{~cm}^{2}\right)$.
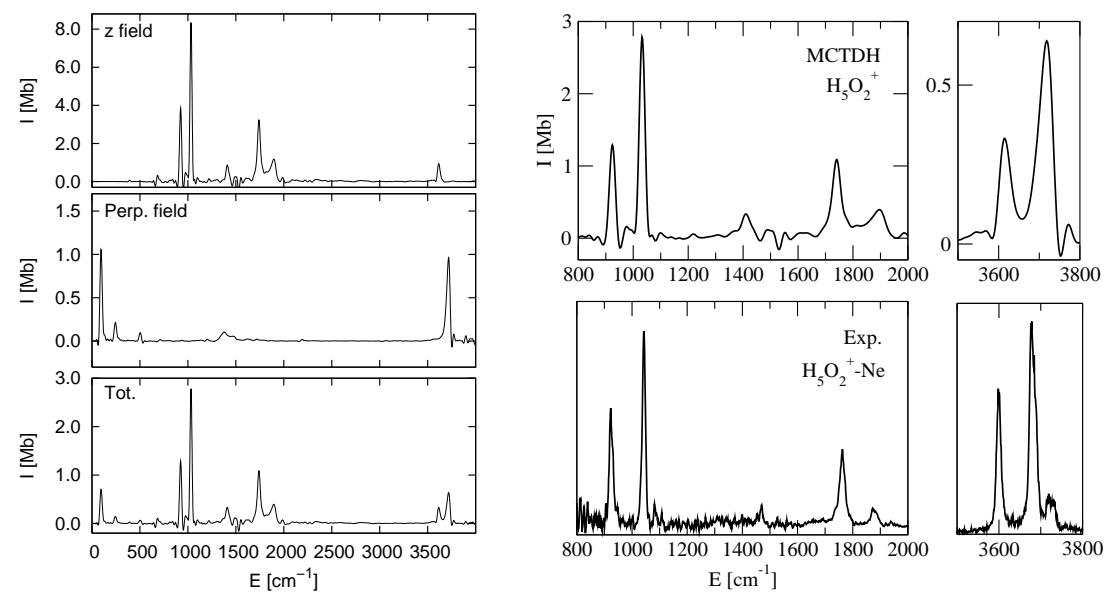

formula [141]:

$$
\left|\left\langle\Phi_{\text {test }} \mid \Psi_{n}\right\rangle\right|^{2}=\frac{\pi}{2 T} R e \int_{0}^{T} e^{i E_{n} t} a(t) \cos \left(\frac{\pi t}{2 T}\right) d t
$$

\subsubsection{Analysis of the middle spectral region}

In the following we apply the described techniques to analyze the spectral region between 800 and $2000 \mathrm{~cm}^{-1}$. This complicated spectral region is shaped by several couplings that involve collective motions of the whole cation. The complication arises from the floppiness and anharmonicity of $\mathrm{H}_{5} \mathrm{O}_{2}^{+}$. Its complete assignment could only be achieved by means of the MCTDH method $[175,214]$ and it constitutes a nice example of the power of this approach to unravel complicated IR signatures. A detailed description of the OH-stretchings' region at about $3600 \mathrm{~cm}^{-1}$ as well as a comprehensive list of all fundamentals and various overtones of all vibrational modes is found in Ref. [175].

The doublet centered at $1000 \mathrm{~cm}^{-1}$ is the most characteristic feature of the IR spectrum of $\mathrm{H}_{5} \mathrm{O}_{2}^{+}$. It is depicted in Fig. 4 (right,top). The highest energy 
Figure 5: Schematic representation of the two most important coupled motions responsible for the doublet peak at $1000 \mathrm{~cm}^{-1}$.

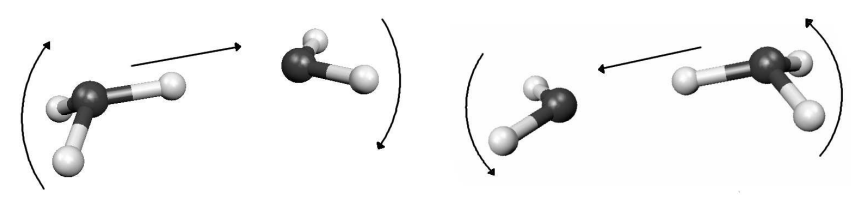

line has been measured to be at $1047 \mathrm{~cm}^{-1}$ while the low energy component appears at $928 \mathrm{~cm}^{-1}$ [208]. These values slightly change to 1042 and $923 \mathrm{~cm}^{-1}$ in the most recent measurements [209], respectively. There is accumulated evidence in the literature that the absorption of the proton-transfer fundamental occurs in the region of $1000 \mathrm{~cm}^{-1}[208,211-213,217]$. This band is the most intense band of the spectrum since the central proton motion along the $z$ axis induces a large change in the dipole-moment of the cation. The low-energy component has been recently assigned by us [214]. The doublet is seen to arise from coupling between the proton-transfer motion, the low frequency water-wagging modes and the water-water stretching motion. In order to obtain a fundamental understanding of the low-energy $\left(\left|\Psi_{d}^{l}\right\rangle\right)$ and high energy $\left(\left|\Psi_{d}^{h}\right\rangle\right)$ components of the doublet, test states were constructed by operating with $\hat{z}$ on the ground state: $\left|\Phi_{1 z}\right\rangle=\hat{z}\left|\Psi_{0}\right\rangle N$, where $N$ is a normalization constant, and by operating with $\left(\hat{R}-R_{0}\right)$ on the third excited wagging state $w_{3}$ : $\left|\Phi_{1 R, w_{3}}\right\rangle=\left(\hat{R}-R_{0}\right)\left|\Psi_{w_{3}}\right\rangle N$. Note that $\left|\Phi_{1 z}\right\rangle$ is characterized by one quantum of excitation in the proton-transfer coordinate whereas $\left|\Phi_{1 R, w_{3}}\right\rangle$ by one quantum in the O-O stretch motion and two quanta in the wagging motion. These two test states were propagated and their auto- and crosscorrelation functions were used for FD analysis, which yielded an energy of $918 \mathrm{~cm}^{-1}$ for $\left|\Psi_{d}^{l}\right\rangle$ and an energy of $1033 \mathrm{~cm}^{-1}$ for $\left|\Psi_{d}^{h}\right\rangle$. These energies are in good accordance with the peaks in Fig. 4 which arise from the propagation of $\left|\Psi_{\mu, 0}\right\rangle$. The spectral intensities were also obtained by FD analysis. The overlaps of the test states to the states making the doublet read: $\left|\left\langle\Phi_{1 z} \mid \Psi_{d}^{l}\right\rangle\right|^{2}=0.09,\left|\left\langle\Phi_{1 R, w_{3}} \mid \Psi_{d}^{l}\right\rangle\right|^{2}=0.83$ and $\left|\left\langle\Phi_{1 z} \mid \Psi_{d}^{h}\right\rangle\right|^{2}=0.46,\left|\left\langle\Phi_{1 R, w_{3}} \mid \Psi_{d}^{h}\right\rangle\right|^{2}=0.10$. One should take into account that these numbers depend on the exact definition of the test states, which is not unique. However, they provide a clear picture of the nature of the doublet: the low-energy band has the largest contribution from the combination of the symmetric stretch and the third excited wagging (see Fig. 3c), whereas the second largest is the proton-transfer motion. For the high-energy band the importance of these two contributions is reversed. Thus, the doublet may be regarded as a Fermi-resonance between two zero-order states which are characterized by $\left(1 R, w_{3}\right)$ and $(1 z)$ excitations, respectively. The reason why the third wagging excitation plays an important role in the proton-transfer doublet 
is understood by inspecting Figs. $3 \mathrm{c}$ and 5 . The probability density of state $w_{3}$ has four maxima, each of which corresponds to a bent conformation of $\mathrm{H}_{2} \mathrm{O}-\mathrm{H}^{+}$ $\left(\mathrm{H}_{3} \mathrm{O}^{+}\right.$character $)$for one of the waters, and a planar conformation $\left(\mathrm{H}_{2} \mathrm{O}\right.$ character) where a lone-pair $\mathrm{H}_{2} \mathrm{O}$ orbital forms a hydrogen bond with the central proton. When the proton oscillates between the two waters, the two conformations exchange their characters accordingly. Thus, the asymmetric wagging mode $\left(w_{3}, 374 \mathrm{~cm}^{-1}\right)$ combines with the water-water stretch motion $(1 R, 550$ $\left.\mathrm{cm}^{-1}[175]\right)$ to reach an energy close to the natural absorption-frequency of the proton transfer, making these motions coupled. The two states of the doublet transform according to the $B_{2}$ irreducible representation of $\mathrm{D}_{2 d}$ and hence couple to the $z$-component of the dipole operator.

The region between the proton-transfer doublet and $2000 \mathrm{~cm}^{-1}$ features couplings related to the $\mathrm{PT}$ and $\mathrm{O}-\mathrm{O}$ stretch motions. The MCTDH spectrum reported in Fig. 4 presents three main absorptions in this range, located at 1411,1741 and $1898 \mathrm{~cm}^{-1}$ and we could assign them to $1 z 1 R$, bu and $1 z 2 R$ excitations, respectively [175]. Here bu stands for bending ungerade, i.e. one water opens while the other closes. This water bending state deserves particular consideration. It couples very strongly to the proton transfer motion and, in fact, its strong brightness is due to this coupling. In a fashion similar to the coupling to the $w_{3}$ wagging motion, as the proton approaches one water molecule the equilibrium value of the $\mathrm{H}-\mathrm{O}-\mathrm{H}$ angle shifts to a larger value because this water molecule acquires more $\mathrm{H}_{3} \mathrm{O}^{+}$character. Conversely, the water molecule at a larger distance of the central proton acquires $\mathrm{H}_{2} \mathrm{O}$ character and the angle $\mathrm{H}-\mathrm{O}-\mathrm{H}$ shifts to lower values. I.e., the concerted motion is similar as depicted in Fig. 5, but with the wagging motion replaced by the bending motion. This coupling shifts the proton-transfer line down and the bending line up by at least $120 \mathrm{~cm}^{-1}$. This estimate was obtained by suppressing the correlation between proton-transfer and bending by setting the number of SPFs to one, for alternatively the proton-transfer mode and the water modes. The frequency of the ungerade bending when decoupled from the proton-transfer motion resembles that of the bare-water bending mode. Thus the shift to a higher frequency is almost entirely due to coupling with the proton-transfer mode.

The reported calculations on $\mathrm{H}_{5} \mathrm{O}_{2}^{+}$were in excellent agreement to the experimental measurements of Refs. [208] and [209] on the messenger-predissociation spectrum of $\mathrm{H}_{5} \mathrm{O}_{2}^{+}$. Ne. Such a remarkable consistency between experiment and theory along the whole spectral range represents, on the one hand, a validation of the underlying potential energy surface of Huang et al. [217] and of the mode-based cluster expansion of the potential used in the quantumdynamical simulations, but is also a clear indication that a suitable set of coordinates was selected to tackle the problem [215].

The fact that the reported simulations were successful in obtaining accurate results for a system of the dimensionality of the protonated water-dimer is to be attributed in great part to the MCTDH algorithm, in which not 
only the expansion coefficients, but also the SPFs, are variationally optimal. For a 15-dimensional system the use of only 4 basis functions per degree of freedom represents of the order of $10^{9}$ configurations. The largest calculations reported here consist of about $10^{7}$ configurations, while already good results are obtained by using as few as $10^{5}$ configurations (Table 7 ). Such an early convergence of the MCTDH method becomes crucial as high-dimensional problems are attempted.

\section{Conclusions}

We reviewed three current approaches to variational solutions to the computation of vibrational energies of polyatomics molecules. Together they span a range from "numerically exact" and general in both the kinetic energy operator and potential to more approximate methods that are more feasible to apply to larger molecules. This "division of labour" is quite typical of the field and is likely to continue in the future.

The numerically exact approach is based on general, so-called polyspherical coordinates. The near exponential scaling of the linear algebra computatations in a direct-product representation of the wave function are avoided by using a combination of multi-dimensional contractions and an iterative eigensolver. The KEO in polyspherical coordinates (Eq. (27)) is general but somewhat more complicated than the Watson normal coordinate KEO. A general polyspherical code that could be used for molecules of different sizes has not been written. Product basis iterative methods have been applied to numerous tetraatomic molecules, however, here we reviewed the application of the contracted basis iterative combination to $\mathrm{CH}_{4}$ using a realistic full dimensional potential energy surface. The method is also being applied to the highly fluxional cation $\mathrm{CH}_{5}^{+}$ again using a full-dimensional PES.

The two other methods approach the vibrational energies of larger molecules quite differently. One is based on the obtaining eigenvalues and eigenfunctions of the Watson Hamiltonian in rectilinear normal modes using Vibrational SelfConsistent Field/Virtual State Configuration Interaction methods. In this approach the key element that permits extension to larger molecules is the nmode representation of the potential. While this representation can be made exact, its power is in providing an accurate representation of the potential (and also the small-in-magnitude vibrational angular momentum terms) with values of $\mathrm{n}$ less than or equal to six. This leads to a "divide-and-conquer" approach to the evaluation of matrix elements of the Hamiltonian. The limitation of normal modes to describe semi-rigid molecules was noted; however, the power of the approach was illustrated for (semi-rigid) $\mathrm{CH}_{4}$ where agreement with benchmark calculations was shown to be excellent. The restriction to semi-rigid molecules can be overcome to an extent by the "Reaction Path Hamiltonian" version of the normal-mode/ n-mode representation approach. 
This power of this approach was illustrated by an application to $\mathrm{CH}_{3} \mathrm{OH}$ which has one large amplitude mode, the floppy periodic torsional motion.

The third approach is distinguished from the two others because it is a time-dependent one. It uses the powerful Multiconfiguration Time-Dependent Hartree method together with a separable product form of the Hamiltonian. This combination permits applications to molecules with 6 and 7 atoms using curvilinear coordinates. The power of the method was illustrated for the floppy 15 degree-of-freedom $\mathrm{H}_{5} \mathrm{O}_{2}^{+}$cation, where the IR spectrum was calculated. As usual with time-dependent approaches the resolution of the spectrum is limited by the length of the propagation time, which cannot be arbitrarily large. In the case of $\mathrm{H}_{5} \mathrm{O}_{2}^{+}$the resolution is of the order of $10-30 \mathrm{~cm}^{-1}$. Nevertheless this was a "triumphant" calculation that was made possible by using a variant of the n-mode representation of the potential instead of the separable product form. This illustrates how various ideas can cross-fertilize overall progress in the field.

Progress in this field has been remarkable. We are now at the stage where we can compute and understand the dynamics of many important molecules. The techniques we have outlined and others related to them can also be used to study the dynamics of dissociating, colliding and reacting molecules. Looking ahead a bit we predict that there will be many interesting applications of these approaches to calculation of vibrational energies of 5, 6, 7-atom polyatomic molecules. With advances in computational power extensions to 10-12 atom molecules are very likely in the near future.

\section{Acknowledgements}

JMB thanks Stuart Carter and Nicholas Handy for wonderful years of collaboration on the code MULTIMODE and much research made possible with that code. He and they thank the Office of Naval Research for financial support.

TC thanks Matthew Bramley and Xiao-Gang Wang who made important contributions to some of the research presented in this paper. His work has been supported by the Natural Sciences and Engineering Research Council of Canada.

HDM thanks F. Gatti for his long-standing cooperation and O. Vendrell for his important contribution to the work on the zundel cation and for help preparing that section of this review. Technical help by M. Eroms on preparing the list of references and financial support by the DFG is gratefully acknowledged.

\section{References}

[1] J. K. G. Watson, Mol. Phys. 15, 479 (1968).

[2] R. J. Whitehead and N. C. Handy, J. Mol. Spec. 55, 356 (1975). 
[3] J. Bowman, Computer Phys. Comm. 51, 225 (1988).

[4] T. J. Lee, editor, Spectrochim Acta, volume 53A, 1997.

[5] S. Carter and N. C. Handy, Mol. Phys. 57, 175 (1986).

[6] S. Carter and N. C. Handy, Computer Phys. Comm. 51, 49 (1988).

[7] N. C. Handy, S. Carter, and S. M. Colwell, Mol. Phys. 96, 477 (1999).

[8] http://www.ccp6.ac.uk/ .

[9] J. Bowman, J. Chem. Phys. 68, 608 (1978).

[10] J. Bowman, K. Christoffel, and F. Tobin, J. Phys. Chem. 83, 905 (1979).

[11] F. Tobin and J. Bowman, Chem. Phys. 47, 151 (1980).

[12] K. Christoffel and J. Bowman, Chem. Phys. Lett. 85, 220 (1982).

[13] J. M. Bowman, J. S. Bittman, and L. B. Harding, J. Chem. Phys. 85, 911 (1986).

[14] B. Gazdy and J. M. Bowman, Adv. Molec Vibration and Collision Dynamics, volume 1B, JAI, Stamford CT, 1991.

[15] H. Romanowski, J. Bowman, and L. Harding, J. Chem. Phys. 82, 4155 (1985).

[16] D. Bégué, N. Gohaud, C. Pouchan, P. Cassam-Chenaï, and J. Liévin, J. Chem. Phys. 127, 164115 (2007).

[17] J. M. Bowman and B. Gazdy, J. Chem. Phys. 94, 454 (1991).

[18] J. C. Light, I. P. Hamilton, and J. V. Lill, J. Chem. Phys. 82, 1400 (1985).

[19] Z. Bačić and J. C. Light, Annu. Rev. Phys. Chem. 40, 469 (1986).

[20] Z. Bačić and J. C. Light, J. Chem. Phys. 86, 3065 (1987).

[21] J. M. Bowman and B. Gazdy, J. Chem. Phys. 93, 1774 (1990).

[22] J. Bowman, B. Gazdy, J. Bentley, T. Lee, and C. Dateo, J. Chem. Phys. 99, 308 (1993).

[23] S. Zou, J. M. Bowman, and A. Brown, J. Chem. Phys. 118, 10012 (2003).

[24] J. C. Tremblay and T. Carrington, Jr., J. Chem. Phys. 125, 094311 (2006). 
[25] I. J. Kozin, M. Law, J. Tennyson, and J. Hutson, J. Chem. Phys. 6, 122 (2005).

[26] J. F. Gaw, A. Willetts, W. H. Green, and N. C. Handy, Adv. Molec Vib and Coll. Dynamics, volume 1B, JAI, Stamford CT, 1991.

[27] E. L. Sibert, Int. Rev. in Phys. Chem 9, 1 (1990).

[28] A. B. McCoy and E. L. Sibert, Canonical van vleck perturbation theory and its application to studies of higly vibrationally excited states of polyatomic molecules, in Dynamics of Molecules and Chemical Reactions, edited by R. E. Wyatt and J. Z. H. Zhang, pages 151-184, New York, 1996, Marcel Dekker.

[29] L. S. Norris, M. A. Ratner, A. E. Roitberg, and R. B. Gerber, J. Chem. Phys. 105, 11261 (1996).

[30] R. B. Gerber, G. M. Chaban, B. Brauer, and Y. Miller, Theory and Applications of Computational Chemistry: The First Forty Years, chapter 9, Elsevier, Amsterdam, 2005.

[31] O. Christiansen, J. Chem. Phys. 119, 5773 (2003).

[32] O. Christiansen, J. Chem. Phys. 120, 2149 (2004).

[33] O. Christiansen and J. Luis, Int. J. Quant. Chem. 104, 667 (2005).

[34] D. Benoit, J. Chem. Phys. 120, 562 (2004).

[35] D. M. Benoit, J. Chem. Phys. 125, 244110 (2006).

[36] Y. Scribano and D. Benoit, J. Chem. Phys. 127, 164118 (2007).

[37] V. Barone, J. Chem. Phys. 122, 014108 (2005).

[38] E. J. Heller, J. Chem. Phys. 62, 1544 (1975).

[39] E. J. Heller, J. Chem. Phys. 64, 63 (1976).

[40] E. A. McCullough and R. E. Wyatt, J. Chem. Phys. 51, 1253 (1969).

[41] E. A. McCullough and R. E. Wyatt, J. Chem. Phys. 54, 3578 (1971).

[42] C. Leforestier et al., J. Comp. Phys. 94, 59 (1991).

[43] R. Kosloff, J. Phys. Chem. 92, 2087 (1988).

[44] D. Neuhauser, J. Chem. Phys. 100, 5076 (1994).

[45] M. R. Wall and D. Neuhauser, J. Chem. Phys. 102, 8011 (1995). 
[46] V. A. Mandelshtam and H. S. Taylor, J. Chem. Phys. 106, 5085 (1997).

[47] V. A. Mandelshtam, J. Chem. Phys. 108, 9999 (1998).

[48] M. Alacid, C. Leforestier, and N. Moiseyev, Chem. Phys. Lett. 305, 258 (1999)

[49] M. H. Beck and H.-D. Meyer, J. Chem. Phys. 109, 3730 (1998).

[50] M. J. Bramley and T. Carrington, Jr., J. Chem. Phys. 99, 8519 (1993).

[51] M. J. Bramley, J. W. Tromp, T. Carrington Jr., and R. C. Corey, J. Chem. Phys. 100, 6175 (1994).

[52] J. C. Light and T. Carrington Jr., Adv. Chem. Phys. 114, 263 (2000).

[53] R. Chen, G. Ma, and H. Guo, J. Chem. Phys. 114, 4764 (2001).

[54] X.-G. Wang and T. Carrington Jr., J. Chem. Phys. 118, 6946 (2003).

[55] T. Carrington Jr., Encyclopedia of Computational Chemistry. Paul von Ragué Schleyer, ed., volume 5, John Wiley \& Sons, Inc., 1998.

[56] X.-G. Wang and T. Carrington, Jr, J. Chem. Phys. 115, 9781 (2001).

[57] S. Carter, S. J. Culik, and J. M. Bowman, J. Chem. Phys. 107, 10458 (1997)

[58] G. Li, S. Wang, C. Rosenthal, and H. Rabitz, J. Math. Chem. 30, 1 (2001).

[59] J. O. Jung and R. B. Gerber, J. Chem. Phys. 105, 10332 (1996).

[60] S. Carter, J. M. Bowman, and B. J. Braams, Chem. Phys. Lett. 342, 636 (2001).

[61] K. Yagi, S. Hirata, and K. Hirao, CA 118, 681 (2007).

[62] G. Rauhut, J. Chem. Phys. 121, 9313 (2004).

[63] H.Fujisaki, K.Yagi, K. Hirao, and J.E.Straub, Chem. Phys. Lett. 443, 6 (2007).

[64] A. L. Kaledin and J. M. Bowman, J. Phys. Chem. A 111, 5593 (2007).

[65] O. F. Alis and H. Rabitz, J. Math. Chem. 29, 127 (2001).

[66] S. Manzhos and T. Carrington, J. Chem. Phys. 125, 084109 (2006).

[67] G. Li, S.-W. Wang, and H. Rabitz, J. Phys. Chem. A 106, 8721 (2002). 
[68] J. Bowman, X. Huang, L. Harding, and S. Carter, Mol. Phys. 104, 33 (2006).

[69] A. Jäckle and H.-D. Meyer, J. Chem. Phys. 104, 7974 (1996).

[70] A. Jäckle and H.-D. Meyer, J. Chem. Phys. 109, 3772 (1998).

[71] M. H. Beck, A. Jäckle, G. A. Worth, and H.-D. Meyer, Phys. Rep 324, 1 (2000).

[72] U. Manthe, J. Chem. Phys. 105, 6989 (1996).

[73] E. Schmidt, Math. Ann. 63, 433 (1906).

[74] S. Manzhos and T. Carrington, J. Chem. Phys. 125, 084109 (2006).

[75] S. Carter, J. M. Bowman, and N. C. Handy, Theor. Chem. Acc. 100, 191 (1998).

[76] J. M. Bowman, S. Carter, and X. Huang, Int. Rev. Phys. Chem. 22, 533 (2003).

[77] J. M. Bowman, S. Carter, and N. C. Handy, Theory and Applications of Computational Chemistry: The First Forty Years., chapter 11, Elsevier, Amsterdam, 2005.

[78] K. Yagi, C. Oyanagi, T. Taketsugu, and K. Hirao, J. Chem. Phys. 118, 1653 (2003).

[79] C. Oyanagi, K. Yagi, T. Taketsugu, and K. Hirao, J. Chem. Phys. 124, 064311 (2006).

[80] T. Hrenar, H.-J. Werner, and G. Rauhut, Phys. Chem. Chem. Phys. 7, 3123 (2005).

[81] G. Rauhut, J. Chem. Phys. 127, 184109 (2007).

[82] S. Carter and J. M. Bowman, J. Chem. Phys. 108, 4397 (1998).

[83] X. Huang, S. Carter, and J. M. Bowman, J. Chem. Phys. 118, 5431 (2003).

[84] C. Leonard, N. C. Handy, S. Carter, and J. M. Bowman, Spectrochimica Acta 58, 825 (2002).

[85] W. H. Miller, N. C. Handy, and J. E. Adams, J. Chem. Phys. 72, 99 (1980).

[86] T. Carrington Jr. and W. H. Miller, J. Chem. Phys. 81, 3942 (184).

[87] S. Carter and N. C. Handy, J. Chem. Phys. 113, 987 (2000). 
[88] D. Tew, N. Handy, S. Carter, S. Irle, and J. Bowman, Mol. Phys. 101, 3513 (2003).

[89] A. B. McCoy, S. C. X. Huang, M. Landeweer, and J. M. Bowman, J. Chem. Phys. 122, 061101 (2005).

[90] S. C. A. B. McCoy, X. Huang and J. M. Bowman, J. Chem. Phys. 123, 064317 (2005).

[91] J. M. Bowman, X. Huang, N. C. Handy, and S. Carter, J. Phys. Chem. A 111, 7317 (2007).

[92] C. Eckart, Phys. Rev. 47, 552 (1935).

[93] H. Wei and T. Carrington, Jr., J. Chem. Phys. 107, 9493 (1997).

[94] H. Wei and T. Carrington, Jr., Chem. Phys. Lett. 287, 289 (1998).

[95] G. A. Natanson, Mol. Phys. 66, 129 (1989).

[96] R. Meyer and H. H. Guenthard, J. Chem. Phys. 49, 1510 (1968).

[97] H. Pickett, J. Chem. Phys. 56, 1715 (1972).

[98] W. H. Press, B. P. Flannery, S. A. Teukolsky, and W. T. Vetterling, Numerical Recipes, Cambridge University Press, 1989.

[99] X. Chapuisat and C. Iung, Phys. Rev. A 45, 6217 (1992).

[100] F. Gatti et al., J. Chem. Phys. 108, 8804 (1998).

[101] C. Iung, F. Gatti, A. Viel, and X. Chapuisat, PCCP 1, 3377 (1999).

[102] M. Mladenović, J. Chem. Phys. 112, 1070 (2000).

[103] M. Mladenović, J. Chem. Phys. 112, 1082 (2000).

[104] X.-G. Wang and T. Carrington, Jr., J. Chem. Phys. 113, 7097 (2000).

[105] B. Podolsky, Phys. Rev. 32, 812 (1928).

[106] A. Nauts and X. Chapuisat, Mol. Phys. 55, 1287 (1985).

[107] X. Chapuisat, A. Nauts, and J.-P. Brunet, Mol. Phys. 72, 1 (1991).

[108] X. Chapuisat, A. Belfhal, and A. Nauts, J. Mol. Spec. 149, 274 (1991).

[109] B. T. Sutcliffe, Current Aspects of Quantum Chemistry, Studies in Theoretical and Physical Chemistry. R. Carbo, ed., volume 21, Elsevier, Amsterdam, 1982.

[110] B. T. Sutcliffe and J. Tennyson, Int. J. Quant. Chem. 39, 183 (1991). 
[111] J. Tennyson and B. T. Sutcliffe, J. Chem. Phys. 77, 4061 (1982).

[112] G. Brocks, A. V. D. Avoird, B. T. Sutcliffe, and J. Tennyson, Mol. Phys. 50, 1025 (1983).

[113] S. S. Xantheas and B. T. Sutcliffe, J. Chem. Phys. 103, 8022 (1995).

[114] N. C. Handy, Mol. Phys. 61, 207 (1987).

[115] F. Gatti, C. Munoz, and C. Iung, J. Chem. Phys. 114, 8275 (2001).

[116] F. Gatti, C. Iung, C. Leforestier, and X. Chapuisat, J. Chem. Phys. 111, 7236 (1999).

[117] X.-G. Wang and T. Carrington, Jr., J. Chem. Phys. 121, 2937 (2004).

[118] R. N. Zare, Angular momentum, Wiley, New York, 1988.

[119] F. Gatti, J. Chem. Phys. 111, 7225 (1999).

[120] R. E. Wyatt, Adv. Chem. Phys. 53, 231 (1989).

[121] H. Köppel, W. Domcke, and L. S. Cederbaum, Adv. Chem. Phys. 57, 59 (1984).

[122] C. Iung and C. Leforestier, J. Chem. Phys. 90, 3198 (1989).

[123] A. McNichols and T. Carrington, Jr., Chem. Phys. Lett. 202, 464 (1993).

[124] J. A. Bentley, J.-P. Brunet, R. E. Wyatt, R. A. Friesner, and C. Leforestier, Chem. Phys. Lett. 161, 393 (1989).

[125] M. J. Bramley and T. Carrington Jr., J. Chem. Phys. 101, 8494 (1994).

[126] D. Neuhauser, J. Chem. Phys. 93, 2611 (1990).

[127] R. B. Lehoucq, S. K. Gray, D.-H. Zhang, and J. C. Light, Computer Phys. Comm. 109, 15 (1998).

[128] H. Y. Mussa and J. Tennyson, J. Chem. Phys. 109, 10885 (1998).

[129] V. A. Mandelshtam and H. S. Taylor, J. Chem. Phys. 102, 7390 (1995).

[130] H.-G. Yu and S. C. Smith, Ber. Bunsenges. Phys. Chem. 101, 400 (1997).

[131] R. Chen and H. Guo, J. Chem. Phys. 108, 6068 (1998).

[132] G. H. Golub and C. F. V. Loan, Matrix Computations, Johns Hopkins University Press, Baltimore, 1989. 
[133] R. A. Friesner, R. E. Wyatt, C. Hempel, and B. Criner, J. Comp. Phys. 64, 220 (1986).

[134] U. Manthe and H. Köppel, J. Chem. Phys. 93, 345 (1990).

[135] J. K. Cullum and R. A. Willoughby, Lanczos Algorithms for Large Symmetric Eigenvalue Computations, volume 1, Birkhäuser, Boston, 1985.

[136] E. Davidson, J. Comp. Phys. 17, 87 (1975).

[137] R. Lehoucq, D. Sorensen, and C. Yang, ARPACK USERS GUIDE: Solution of Large Scale Eigenvalue Problems by Implicitly Restarted Arnoldi Methods, SIAM, Philadelphia, 1998.

[138] S. Huang and T. Carrington, Jr., J. Chem. Phys. 112, 8765 (2000).

[139] S.-W. Huang and T. Carrington Jr., Chem. Phys. Lett. 312, 311 (1999).

[140] P.-N. Roy and T. Carrington Jr., J. Chem. Phys. 103, 5600 (1995).

[141] H.-D. Meyer, F. Le Quéré, C. Léonard, and F. Gatti, Chem. Phys. 329, 179 (2006).

[142] H.-G. Yu and J. T. Muckerman, J. Mol. Struct. (Theochem) 214, 11 (2002).

[143] G. Czako, T. Furtenbacher, A. G. Csaszar, and V. Szalay, Mol. Phys. 102, 2411 (2004)

[144] Z. Bačić and J. C. Light, Ann. Rev. Phys. Chem. 40, 469 (1989).

[145] J. R. Henderson and J. Tennyson, Chem. Phys. Lett. 173, 133 (1990).

[146] X. T. Wu, A. B. McCoy, and E. F. Hayes, J. Chem. Phys. 110, 2354 (1999).

[147] Y. Qiu, J. Z. H. Zhang, and Z. Bačić, J. Chem. Phys. 108, 4804 (1998).

[148] A. Bahel and Z. Bačić, J. Chem. Phys. 111, 11164 (1999).

[149] M. Mladenović, Spectrochim. Acta Part A 58, 809 (2002).

[150] M. Mladenović, Spectrochim. Acta Part A 58, 795 (2002).

[151] D. Luckhaus, J. Chem. Phys. 113, 1329 (2000).

[152] M. J. Bramley and N. C. Handy, J. Chem. Phys. 98, 1378 (1993).

[153] S. Carter and N. C. Handy, Mol. Phys. 100, 681 (2002). 
[154] J. Koput, S. Carter, and N. Handy, J. Chem. Phys. 115, 8345 (2001).

[155] R. A. Friesner, J. Bentley, M. menou, and C. Leforestier, J. Chem. Phys. 99, 324 (1993).

[156] A. Viel and C. Leforestier, J. Chem. Phys. 112, 1212 (2000).

[157] X.-G. Wang and J. T. Carrington, J. Chem. Phys. 117, 6923 (2002).

[158] X.-G. Wang and T. Carrington, Jr, J. Chem. Phys. 119, 101 (2003).

[159] H.-G. Yu, J. Chem. Phys. 117, 2030 (2002).

[160] H.-G. Yu, J. Chem. Phys. 117, 8190 (2002).

[161] H.-S. Lee and J. C. Light, J. Chem. Phys. 118, 3458 (2003).

[162] H.-S. Lee and J. C. Light, J. Chem. Phys. 120, 4626 (2004).

[163] Y. Wang, S. Carter, B. J. Braams, and J. M. Bowman, J. Chem. Phys. 128, 071101 (2008).

[164] S. Carter, N. C. Handy, and J. M. Bowman, to be published .

[165] X. T. Wu and E. F. Hayes, J. Chem. Phys. 107, 2705 (1997).

[166] H.-D. Meyer, U. Manthe, and L. S. Cederbaum, Chem. Phys. Lett. 165, 73 (1990).

[167] U. Manthe, H.-D. Meyer, and L. S. Cederbaum, J. Chem. Phys. 97, 3199 (1992).

[168] H.-D. Meyer and G. A. Worth, Theor. Chem. Acc. 109, 251 (2003).

[169] M. H. Beck and H.-D. Meyer, J. Chem. Phys. 114, 2036 (2001).

[170] K. C. Kulander, editor, Time-dependent methods for quantum dynamics, Elsevier, Amsterdam, 1991.

[171] R. Kosloff, Ann. Rev. Phys. Chem. 45, 145 (1994).

[172] M. H. Beck and H.-D. Meyer, Z. Phys. D 42, 113 (1997).

[173] R. Schinke, Photodissociation Dynamics, Cambridge University Press, Cambridge, 1993.

[174] G. G. Balint-Kurti, R. N. Dixon, and C. C. Marston, J. Chem. Soc., Faraday Trans. 86, 1741 (1990).

[175] O. Vendrell, F. Gatti, and H.-D. Meyer, J. Chem. Phys. 127, 184303 (2007). 
[176] U. Manthe, H.-D. Meyer, and L. S. Cederbaum, J. Chem. Phys. 97, 9062 (1992).

[177] V. Engel, Chem. Phys. Lett. 189, 76 (1992).

[178] A. Raab, G. Worth, H.-D. Meyer, and L. S. Cederbaum, J. Chem. Phys. 110, 936 (1999).

[179] C. Cattarius, G. A. Worth, H.-D. Meyer, and L. S. Cederbaum, J. Chem. Phys. 115, 2088 (2001).

[180] S. Mahapatra, G. A. Worth, H. D. Meyer, L. S. Cederbaum, and H. Köppel, J. Phys. Chem. A 105, 5567 (2001).

[181] H. Köppel, M. Döscher, I. Baldea, H.-D. Meyer, and P. G. Szalay, J. Chem. Phys. 117, 2657 (2002).

[182] E. V. Gromov et al., J. Chem. Phys. 121, 4585 (2004).

[183] A. Markmann et al., J. Chem. Phys. 123, 204310 (2005).

[184] F. Gatti, M. H. Beck, G. A. Worth, and H.-D. Meyer, PCCP 3, 1576 (2001).

[185] F. Richter, P. Rosmus, F. Gatti, and H.-D. Meyer, J. Chem. Phys. 120, $6072(2004)$.

[186] R. Kosloff and H. Tal-Ezer, Chem. Phys. Lett. 127, 223 (1986).

[187] J. Hinze, J. Chem. Phys. 59, 6424 (1973).

[188] K. Drukker and S. Hammes-Schiffer, J. Chem. Phys. 107, 363 (1997).

[189] E. A. Hylleraas and B. Undheim, Z. Phys. 65, 759 (1930).

[190] J. K. L. MacDonald, Phys. Rev. 43, 830 (1933).

[191] F. Richter, F. Gatti, C. Léonard, F. Le Quéré, and H.-D. Meyer, J. Chem. Phys. 127, 164315 (2007).

[192] G. A. Worth, M. H. Beck, A. Jäckle, and H.-D. Meyer, The Heidelberg MCTDH Package: A set of programs for multi-dimensional quantum dynamics. User's Guide, Version 8.4, 2007

(The User's Guide can be downloded from the URL: http://mctdh.unihd.de/).

[193] F. Richter, M. Hochlaf, P. Rosmus, F. Gatti, and H.-D. Meyer, J. Chem. Phys. 120, 1306 (2004).

[194] H.-G. Yu, J. Chem. Phys. 120, 2270 (2004). 
[195] F. T. Smith, Phys. Rev. 179, 111 (1969).

[196] H. Wei and T. Carrington Jr., J. Chem. Phys. 97, 3029 (1992).

[197] J. Echave and D. C. Clary, Chem. Phys. Lett. 190, 225 (1992).

[198] D. T. Colbert and W. H. Miller, J. Chem. Phys. 96, 1982 (1992).

[199] X.-G. Wang and T. Carrington, Jr., J. Chem. Phys. 114, 1473 (2001).

[200] R. Chen and H. Guo, J. Chem. Phys. 114, 1467 (2001).

[201] X.-G. Wang and T. Carrington, Jr., J. Chem. Phys. 123, 154303 (2005).

[202] D. W. Schwenke and H. Partridge, Spectrochim. Acta Part A 57, 887 (2001).

[203] X. Wang and T. Carrington Jr., J. Chem. Phys. xxx, in press (2008).

[204] X. H. J. Wu, S. Carter, and J. M. Bowman, Chem. Phys. Lett. 426, 285 (2006).

[205] K. R. Asmis et al., Science 299, 1375 (2003).

[206] T. D. Fridgen, T. B. McMahon, L. MacAleese, J. Lemaire, and P. Maitre, J. Phys. Chem. A 108, 9008 (2004).

[207] J. M. Headrick, J. C. Bopp, and M. A. Johnson, J. Chem. Phys. 121, 11523 (2004).

[208] N. I. Hammer et al., J. Chem. Phys. 122, 244301 (2005).

[209] L. McCunn, J. Roscioli, M. Johnson, and A. McCoy, Journal of Physical Chemistry B 112, 321 (2008).

[210] M. V. Vener, O. Kühn, and J. Sauer, J. Chem. Phys. 114, 240 (2001).

[211] J. Dai, Z. Bacic, X. C. Huang, S. Carter, and J. M. Bowman, J. Chem. Phys. 119, 6571 (2003).

[212] J. Sauer and J. Dobler, Chem. Phys. Chem. 6, 1706 (2005).

[213] M. Kaledin, A. L. Kaledin, and J. M. Bowman, J. Phys. Chem. A 110, 2933 (2006).

[214] O. Vendrell, F. Gatti, and H.-D. Meyer, Angew. Chem. Int. Ed. 46, 6918 (2007).

[215] O. Vendrell, F. Gatti, D. Lauvergnat, and H.-D. Meyer, J. Chem. Phys. 127, 184302 (2007). 
[216] K. Giese and O. Kühn, J. Chem. Phys. 123, 054315 (2005).

[217] X. Huang, B. J. Braams, and J. M. Bowman, J. Chem. Phys. 122, 044308 (2005).

[218] A. B. McCoy, X. Huang, S. Carter, M. Y. Landeweer, and J. M. Bowman, J. Chem. Phys. 122, 061101 (2005). 
TABLE I: Vibrational energy levels of $\mathrm{CH}_{4}$ in $\mathrm{cm}^{-1}$.

\begin{tabular}{|c|c|c|c|c|c|c|c|c|c|c|c|}
\hline \multicolumn{2}{|c|}{$\begin{array}{l}\left(n_{1} n_{3}\right)\left(n_{2} n_{4}\right) \text { Basis I } \\
\text { Sym }\end{array}$} & \multirow{2}{*}{$\begin{array}{l}\text { Basis II } \\
9691.534\end{array}$} & \multirow{2}{*}{$\begin{array}{c}\text { Basis } \\
\text { I - II } \\
0.000\end{array}$} & \multicolumn{2}{|c|}{$\begin{array}{l}\left(n_{1} n_{3}\right)\left(n_{2} n_{4}\right) \text { Basis I } \\
\text { Sym }\end{array}$} & \multirow{2}{*}{$\begin{array}{l}\text { Basis II } \\
5236.452\end{array}$} & \multirow{2}{*}{$\begin{array}{c}\text { Basis } \\
\text { I - II } \\
1.286\end{array}$} & \multicolumn{2}{|c|}{$\begin{array}{l}\left(n_{1} n_{3}\right)\left(n_{2} n_{4}\right) \text { Basis I } \\
\text { Sym }\end{array}$} & \multirow{2}{*}{$\begin{array}{l}\text { Basis II } \\
5881.609\end{array}$} & \multirow{2}{*}{$\begin{array}{r}\text { Basis } \\
\text { I - II } \\
4.540\end{array}$} \\
\hline (00)(00) A1 & 9691.534 & & & 00)(04) F1 & 5237.738 & & & (00)(31) F1 & 5886.149 & & \\
\hline$(00)(01) \mathrm{F} 2$ & 1311.742 & 1311.742 & 0.000 & $(00)(04) \mathrm{A} 1$ & 5248.437 & 5247.146 & 1.291 & $(00)(31) \mathrm{F} 2$ & 5900.481 & 5896.068 & 1.413 \\
\hline E & 1533.24 & 33.245 & 000 & 3) F2 & 5383.087 & 5375.661 & 7.426 & 1) F1 & & & .441 \\
\hline$(00)(02) \mathrm{A} 1$ & 2589.774 & 589.770 & .004 & (00)(13) F1 & 5401.639 & 5394.211 & 7.428 & $(10)(20) \mathrm{A} 1$ & 5933.239 & 5933.028 & 0.211 \\
\hline 2) $F 2$ & 2616.238 & 16.235 & & 3) $\mathrm{E}$ & & & 6.880 & 20) E & & & .268 \\
\hline$(00)(02) \mathrm{E}$ & 2627.295 & 627.292 & .003 & $(00)(13) \mathrm{F} 2$ & 5440.667 & 5434.215 & & $(02)(00) \mathrm{A} 1$ & & & 0.132 \\
\hline (00)(11) F2 & 2831.528 & 831.519 & 0.009 & (00)(13) F1 & 5448.072 & & 6.654 & $(02)(00) \mathrm{F} 2$ & 5993.506 & & 0.050 \\
\hline 11) $\mathrm{F} 1$ & 2846.914 & 66 & 008 & $0)(13) \mathrm{F} 2$ & 5455.056 & 5448.486 & & $(00) \mathrm{E}$ & & & 013 \\
\hline$(10)(00) \mathrm{A} 1$ & 2913.707 & 913.708 & -0.001 & $(00)(13) \mathrm{F} 1$ & 5473.525 & 5467.126 & 6.399 & $(01)(20) \mathrm{F} 2$ & 6048.215 & 6047.968 & 0.247 \\
\hline$(01)(00) \mathrm{F} 2$ & 3013.600 & 013.601 & & $(10)(02) \mathrm{A} 1$ & 5493.222 & & & 1)(20) F1 & & & 0.410 \\
\hline$(00)(20) \mathrm{A} 1$ & 3063.489 & 3063484 & 0.005 & $(10)(02) \mathrm{F} 2$ & 5521.205 & 5520.923 & 0.282 & $(01)(20) \mathrm{F} 2$ & 6060.059 & 6059.555 & 0.504 \\
\hline 0) $\mathrm{E}$ & 3065.010 & 065.006 & 004 & 10)(02) E & 5533.970 & 5533.680 & 0.290 & $(00)(40) \mathrm{A} 1$ & 238 & & 1.733 \\
\hline$(00)(03) \mathrm{F} 2$ & 3874.749 & 3874.735 & 0.014 & $(01)(02) \mathrm{F} 2$ & 5585.364 & 5584.988 & 0.376 & $(00)(40) \mathrm{E}$ & 6123.004 & & 1.801 \\
\hline$(00)(03) \mathrm{A} 1$ & & & .013 & (01)(02) A1 & & & & $(00)(40) \mathrm{E}$ & & & 1.887 \\
\hline$(00)(03) \mathrm{F} 1$ & 3924.090 & 924.078 & .012 & $(01)(02) \mathrm{F} 2$ & 5612.274 & 5611.786 & & $(00)(05) \mathrm{F} 2$ & 6397.920 & 6397.6 & 0.305 \\
\hline$(00)(03) \mathrm{F} 2$ & & 935.324 & & 01)(02) F1 & & & & $(00)(05) \mathrm{A} 1$ & & & 0.329 \\
\hline$(00)(12) \mathrm{E}$ & 4104.480 & 104.436 & .044 & $(01)(02) \mathrm{E}$ & & 5612.708 & 0.940 & $(05) \mathrm{F} 1$ & & & 0.323 \\
\hline$(00)(12) \mathrm{F} 1$ & 4131.305 & 4131.260 & 0.045 & $(00)(22)$ A1 & 5620.131 & 5614.482 & & $(00)(05) \mathrm{F} 2$ & 6469.634 & 6469.299 & 0.335 \\
\hline 2) $\mathrm{A} 1$ & & & 042 & 01)(02) F1 & & & 3.729 & $(05) \mathrm{E}$ & & & 0.360 \\
\hline$(00)(12) \mathrm{F} 2$ & 4144.878 & 4144.833 & 0.045 & $(01)(02) \mathrm{F} 2$ & 5625.321 & 5623.051 & 2.270 & $(00)(05) \mathrm{F} 2$ & 6524.390 & 6524.038 & 0.352 \\
\hline 2) $\mathrm{E}$ & & & 0.039 & $(00)(22) \mathrm{E}$ & & 562 & 0.634 & (05) F1 & & & 0.375 \\
\hline$(00)(12)$ A2 & 4164.360 & 4164.322 & 0.038 & $(00)(22) \mathrm{F} 2$ & 5651.123 & 5645.308 & & $(00)(05) \mathrm{F} 2$ & 6556.638 & & 0.389 \\
\hline$(10)(01) \mathrm{F} 2$ & 4221.845 & 221.844 & 0.001 & $(00)(22) \mathrm{E}$ & 5662.914 & 5657.189 & 5.725 & $(00)(14) \mathrm{E}$ & 6639.953 & 6635.194 & 4.759 \\
\hline$(01)(01) \mathrm{F} 2$ & & & .000 & $(00)(22) \mathrm{F} 1$ & & 5658.373 & 5.889 & (14) F1 & & & 5.107 \\
\hline$(01)(01) \mathrm{E}$ & 4317.587 & 4317.585 & 0.002 & $(00)(22) \mathrm{A} 2$ & 5672.243 & 5666.608 & 5.635 & $(00)(14) \mathrm{A} 1$ & 6677.726 & 6672.514 & 5.212 \\
\hline 01) $\mathrm{F} 1$ & 4317.830 & 317.828 & 002 & $(00)(22) \mathrm{F} 2$ & & & 5.878 & (14) F2 & & & 5.289 \\
\hline$(01)(01) \mathrm{A} 1$ & 4318.421 & 4318.418 & 2002 & $(00)(22) \mathrm{A} 1$ & 5689.821 & 5684.183 & 5.638 & $(00)(14) \mathrm{E}$ & 6702.196 & & 5.304 \\
\hline$(00)(21) \mathrm{F} 2$ & 4350.100 & 4350.067 & 0.033 & $(00)(22) \mathrm{E}$ & 5699.177 & 5693.519 & & $(00)(14) \mathrm{A} 2$ & 6704.281 & 6698.831 & 5.450 \\
\hline$(00)(21) \mathrm{F} 1$ & 4364.739 & 4364.709 & 0.030 & $(10)(11) \mathrm{F} 2$ & 5725.662 & 5725.122 & 0.540 & $(00)(14) \mathrm{F} 2$ & 6737.709 & 6731.722 & 5.987 \\
\hline$(00)(21) \mathrm{F} 2$ & 4379.765 & & 0.030 & $(10)(11) \mathrm{F} 1$ & 5743.523 & 5743.057 & & $(00)(14) \mathrm{F} 1$ & 6741.714 & & 5.875 \\
\hline$(10)(10) \mathrm{E}$ & 4432.221 & 4432.220 & 0.001 & $(20)(00)$ A1 & 5782.245 & 5782.176 & 0.069 & $(00)(14) \mathrm{E}$ & 6750.148 & 6743.877 & 6.271 \\
\hline$(01)(10) \mathrm{F} 1$ & 4531.370 & & > & $(01)(11) \mathrm{F} 2$ & & & & $(00)(14) \mathrm{F} 2$ & & & 6.090 \\
\hline$(01)(10) \mathrm{F} 2$ & 4537.823 & 4537.821 & & $(01)(11) \mathrm{F} 1$ & & & & $(00)(14) \mathrm{A} 1$ & & & 5.595 \\
\hline$(00)(30) \mathrm{E}$ & 4591.935 & 4591.922 & 0012 & $(01)(11) \mathrm{E}$ & 5828.639 & 5827.837 & 0.802 & $(00)(14) \mathrm{A} 2$ & 6766.866 & 6760.892 & 5.974 \\
\hline$(00)(30)$ A2 & 4595.171 & 4595.158 & & $(01)(11) \mathrm{A} 1$ & & & & $(10)(03) \mathrm{F} 2$ & & 6769.154 & 3.334 \\
\hline$(00)(30) \mathrm{A} 1$ & 4595.439 & 4595.426 & 0.013 & $(01)(11) \mathrm{E}$ & 5838.452 & 5837.924 & 0.528 & $(00)(14) \mathrm{F} 1$ & 6775.326 & 6772.211 & 3.115 \\
\hline$(00)(04) \mathrm{A} 1$ & 5129.718 & 5128.288 & 1.430 & $(01)(11)$ A2 & 5838.477 & 5837.996 & 0.481 & $(00)(14) \mathrm{E}$ & 6786.619 & 6780.375 & 6.244 \\
\hline$(00)(04) \mathrm{F} 2$ & 5150.563 & 5149.196 & 1.367 & $(01)(11) \mathrm{F} 2$ & 5839.469 & 5838.970 & 0.499 & $(10)(03) \mathrm{A} 1$ & 6811.533 & 6810.945 & 0.588 \\
\hline$(00)(04) \mathrm{E}$ & 5175.422 & 5174.008 & 1.414 & (01)(11) F1 & 5842.714 & 5842.174 & 0.540 & $(10)(03) \mathrm{F} 1$ & 6824.713 & 6824.309 & 0.404 \\
\hline$(00)(04) \mathrm{F} 2$ & 5216.979 & 5215.683 & 1.296 & $(11)(00) \mathrm{F} 2$ & 5853.956 & 5853.588 & 0.368 & $(10)(03) \mathrm{F} 2$ & 6836.624 & 6836.269 & 0.355 \\
\hline$(00)(04) \mathrm{E}$ & 5236.081 & 5234.764 & 1.317 & $(00)(31) \mathrm{F} 2$ & 5872.466 & 5868.167 & 4.299 & $(01)(03) \mathrm{F} 2$ & 6858.634 & 6858.438 & 0.196 \\
\hline
\end{tabular}


TABLE I: Vibrational energy levels of $\mathrm{CH}_{4}$ in $\mathrm{cm}^{-1}$ (continued).

\begin{tabular}{|c|c|c|c|c|c|c|c|c|c|c|c|}
\hline \multicolumn{2}{|c|}{$\begin{array}{l}\left(n_{1} n_{3}\right)\left(n_{2} n_{4}\right) \text { Basis I } \\
\text { Sym }\end{array}$} & \multirow{2}{*}{$\begin{array}{l}\text { Basis II } \\
6862.290\end{array}$} & \multirow{2}{*}{$\begin{array}{c}\text { Basis } \\
\text { I - II } \\
0.347\end{array}$} & \multicolumn{2}{|c|}{$\begin{array}{l}\left(n_{1} n_{3}\right)\left(n_{2} n_{4}\right) \text { Basis I } \\
\text { Sym }\end{array}$} & \multirow{2}{*}{$\begin{array}{l}\text { Basis II } \\
7131.695\end{array}$} & \multirow{2}{*}{$\begin{array}{c}\text { Basis } \\
\text { I - II } \\
1.609\end{array}$} & \multicolumn{2}{|c|}{$\begin{array}{l}\left(n_{1} n_{3}\right)\left(n_{2} n_{4}\right) \text { Basis I } \\
\text { Sym }\end{array}$} & \multirow{2}{*}{$\begin{array}{l}\text { Basis II } \\
7364.377\end{array}$} & \multirow{2}{*}{$\begin{array}{l}\text { Basis } \\
\text { I - II } \\
0.410\end{array}$} \\
\hline (01)(03) F1 & 6862.637 & & & $(00)(32) \mathrm{E}$ & 7133.304 & & & $(01)(21) \mathrm{F} 1$ & 7364.787 & & \\
\hline (01)(03) E & 6862.856 & 6862.400 & 0.456 & $(01)(12) \mathrm{F} 1$ & 7136.597 & 7136.117 & 0.480 & (11)(10) F1 & 7365.047 & 7364.825 & 0.222 \\
\hline$(01)(03) \mathrm{A} 1$ & 6864.172 & 363.915 & 257 & 01)(12) F2 & 7139.734 & 7137.288 & 2.446 & $(11)(10) \mathrm{F} 2$ & 7368.767 & 7368.408 & .359 \\
\hline (00)(23) F2 & 6887.011 & 6882.949 & 4.062 & (00)(32) A1 & 7140.679 & 7139.156 & 1.523 & $(00)(41) \mathrm{F} 2$ & 7391.612 & 7389.133 & 2.479 \\
\hline$(01)(03) \mathrm{F} 2$ & 6896.894 & 396.508 & .386 & 1)(12) A2 & 7147.895 & 7144.203 & 3.692 & $(00)(41) \mathrm{F} 1$ & 7404.788 & 7402.065 & 2.723 \\
\hline$(00)(23) \mathrm{F} 1$ & 6907.072 & 6902.987 & 4.085 & $(01)(12) \mathrm{F} 1$ & 7149.514 & 7148.974 & 0.540 & $(00)(41) \mathrm{F} 2$ & 7418.752 & 7416.101 & 2.651 \\
\hline (01)(03) E & 6907.488 & 907.256 & 0.232 & 11)(01) A1 & 7150.344 & 7150.077 & 0.267 & $(00)(41) \mathrm{F} 1$ & 7432.489 & & 2.823 \\
\hline$(01)(03) \mathrm{F} 2$ & 6910.086 & 6909.769 & 0.317 & (01) F2 & 7151.121 & 7150.926 & 0.195 & $(10) \mathrm{E}$ & 7440.532 & 7440.358 & 0.174 \\
\hline (01)(03) F1 & 6915.098 & & .799 & 11)(01) F1 & 7157.548 & 7156.866 & 0.682 & $(00)(41) \mathrm{F} 2$ & 7446.767 & & 2.600 \\
\hline$(01)(03)$ A2 & 6918.017 & 6917.476 & 0.541 & )(01) E & 7158.182 & 7157.945 & 0.237 & $(10)(30) \mathrm{A} 2$ & 7465.976 & 7465.778 & 0.198 \\
\hline$(01)(03) \mathrm{F} 1$ & 6921.095 & 917.713 & 3.382 & (00)(32) F1 & 7168.870 & 7165.230 & 3.640 & $(10)(30) \mathrm{A} 1$ & 7466.304 & & 0.196 \\
\hline$(00)(23) \mathrm{F} 2$ & 6922.285 & 917.880 & 4.405 & $(00)(32) \mathrm{E}$ & 7181.075 & 7176.832 & 4.243 & (10)(30) E & 7477.209 & & 0.099 \\
\hline$(01)(03)$ A1 & 6922.779 & 6922.210 & 0.569 & $(00)(32) \mathrm{F} 2$ & 7181.629 & 7177.360 & 4.269 & $(02)(10) \mathrm{F} 2$ & 7498.828 & & 0.047 \\
\hline$(01)(03) \mathrm{F} 2$ & 6925.417 & 924.907 & .510 & $(00)(32) \mathrm{A} 1$ & 7190.048 & 7185.795 & 4.253 & (10) F1 & 7501.091 & 7501.022 & 0.069 \\
\hline$(01)(03) \mathrm{E}$ & 6927.081 & 6926.541 & 0.540 & $(00)(32) \mathrm{F} 1$ & 7193.320 & 7189.028 & 4.292 & $(02)(10)$ A2 & 7533.954 & 7533.941 & 0.013 \\
\hline$(00)(23) \mathrm{E}$ & 6955.225 & 949.996 & .229 & (00)(32) F2 & 7203.317 & & 3.850 & $(02)(10) \mathrm{E}$ & & & 0.020 \\
\hline$(00)(23) \mathrm{A} 1$ & 6956.380 & 6951.302 & 5.078 & $(00)(32) \mathrm{E}$ & 7205.049 & 7200.832 & 4.217 & $(02)(10) \mathrm{A} 1$ & 7546.935 & 7546.912 & 0.023 \\
\hline$(00)(23) \mathrm{F} 2$ & 6957.415 & & 5.436 & $(10)(21) \mathrm{F} 2$ & & & & $(01)(30) \mathrm{F} 1$ & & & 0.185 \\
\hline$(00)(23) \mathrm{F} 1$ & 6962.104 & 956.920 & .184 & $(00)(32) \mathrm{E}$ & 7230.825 & 7226.625 & 4.200 & (30) F2 & 7570.195 & & 0.253 \\
\hline$(00)(23) \mathrm{F} 1$ & 6966.013 & 6960.834 & 5.179 & $(00)(32) \mathrm{A} 2$ & 7234.569 & 7230.150 & 4.419 & $(01)(30) \mathrm{F} 1$ & 7575.798 & 7575.488 & 0.310 \\
\hline$(00)(23) \mathrm{F} 2$ & 6978.751 & 973.629 & .122 & $(02)(01) \mathrm{F} 2$ & & & 0.245 & $(01)(30) \mathrm{F} 2$ & & & 0.316 \\
\hline$(00)(23) \mathrm{F} 1$ & 6988.818 & 6983.620 & 5.198 & (10)(21) F1 & 7245.156 & 7244.629 & 0.527 & $(00)(06) \mathrm{A} 1$ & 7642.658 & 7636.784 & 5.874 \\
\hline$(10)(12) \mathrm{E}$ & 6991.853 & 991.115 & 738 & $(21) \mathrm{F} 2$ & 7266.831 & 7266.403 & 0.428 & $(50) \mathrm{E}$ & 7647.223 & 7647 & 0.168 \\
\hline$(00)(23) \mathrm{F} 2$ & 7009.358 & 7004.032 & 5.326 & $(02)(01) \mathrm{E}$ & 7285.582 & 7285.540 & 0.042 & $(00)(50) \mathrm{A} 2$ & 7650.730 & 7650.557 & 0.173 \\
\hline (10)(12) F1 & 7021.853 & 7020.912 & 0.941 & $(02)(01) \mathrm{F} 1$ & 7286.071 & 7286.009 & & $(00)(50) \mathrm{A} 1$ & 7651.702 & 7651.526 & 0.176 \\
\hline (10)(12) A1 & 7025.586 & 7024.749 & 0.837 & $(20)(10) \mathrm{E}$ & 7287.330 & 7287.225 & 0.105 & $(00)(06) \mathrm{F} 2$ & 7658.691 & 7652.860 & 5.831 \\
\hline (10)(12) F2 & 7035.699 & 7034.960 & 0.739 & $(02)(01)$ A1 & 7289.339 & 7289.273 & 0.066 & $(00)(50) \mathrm{E}$ & 7659.222 & 7659.034 & 0.188 \\
\hline$(10)(12) \mathrm{E}$ & 7047.090 & 7046.377 & 0.713 & $(02)(01) \mathrm{F} 2$ & & & & $(00)(06) \mathrm{E}$ & 7696.374 & & 6.209 \\
\hline$(10)(12)$ A2 & 7058.012 & & 0.653 & $(01)(21) \mathrm{F} 1$ & 7318.898 & & & $(00)(06) \mathrm{F} 2$ & 7725.154 & & 6.360 \\
\hline$(20)(01) \mathrm{F} 2$ & 7080.505 & 7080.231 & 0.274 & $(01)(21) \mathrm{F} 2$ & 7324.018 & 7323.840 & & $(00)(06) \mathrm{F} 1$ & 7756.934 & 7750.604 & 6.330 \\
\hline$(01)(12) \mathrm{F} 1$ & 7082.115 & 7081.781 & 0.334 & $(02)(01) \mathrm{F} 2$ & 7328.089 & 7327.973 & 0.116 & $(00)(06) \mathrm{E}$ & 7763.753 & 7757.243 & 6.510 \\
\hline$(01)(12) \mathrm{F} 2$ & 7094.235 & & 0.366 & $(02)(01) \mathrm{F} 1$ & 7328.248 & & 0.079 & $(00)(06) \mathrm{A} 1$ & 7774.669 & & 6.354 \\
\hline$(01)(12) \mathrm{E}$ & 7104.536 & 7104.099 & & $(01)(21) \mathrm{A} 1$ & 7339.081 & 7338.464 & & $(00)(06) \mathrm{F} 1$ & 7832.315 & 7824.863 & 7.452 \\
\hline$(01)(12)$ A2 & 7111.757 & 1111.400 & 0.324 & $(01)(21) \mathrm{E}$ & 7339.241 & & 0.583 & $(00)(06) \mathrm{A} 1$ & & & 7.590 \\
\hline$(01)(12) \mathrm{F} 2$ & 7113.200 & 7112.724 & 0.476 & $(01)(21) \mathrm{F} 2$ & & & & $(00)(06) \mathrm{F} 2$ & 7835.779 & 7828.485 & 7.294 \\
\hline$(01)(12) \mathrm{F} 1$ & 7115.247 & 7114.710 & 0.537 & $(01)(21) \mathrm{F} 1$ & 7340.921 & 7340.646 & 0.275 & $(00)(06) \mathrm{A} 2$ & 7861.814 & 7855.028 & 6.786 \\
\hline$(01)(12) \mathrm{F} 2$ & 7118.843 & 7118.474 & & $(01)(21) \mathrm{A} 2$ & & & & $(00)(06) \mathrm{F} 2$ & 7870.586 & & 6.750 \\
\hline$(01)(12) \mathrm{A} 1$ & 7121.894 & 7121.111 & 0.783 & $(01)(21) \mathrm{E}$ & & 7348.659 & 0.586 & $(00)(06) \mathrm{E}$ & 7874.747 & 7867.861 & 6.886 \\
\hline$(01)(12) \mathrm{F} 2$ & 7128.653 & 7126.531 & 2.122 & (01)(21) A1 & 7356.587 & 7356.241 & 0.346 & & & & \\
\hline$(01)(12) \mathrm{E}$ & 7128.876 & 7128.113 & 0.763 & $(01)(21) \mathrm{E}$ & 7357.761 & 7357.296 & 0.465 & & & & \\
\hline (01)(12) F1 & 7129.014 & 7128.441 & 0.573 & $(01)(21) \mathrm{F} 2$ & 7360.835 & 7360.439 & 0.396 & & & & \\
\hline
\end{tabular}

